\title{
IDENTIFICATION CODES FOR ORGANIZATIONS LISTED IN COMPUTERIZED DATA SYSTEMS OF THE U.S. GEOLOGICAL SURVEY
}

By Melvin D.Edwards and Beverly M. Josefson

U.S. GEOLOGICAL SURVEY

Open-File Report 82-921

(Supersedes Open-File Report 81-904) 


\section{UNITED STATES DEPARTMENT OF THE INTERIOR \\ JAMES G. WATT, Secretary}

GEOLOGICAL SURVEY

Dallas L. Peck, Director

For additional information write to:

Copies of this report can be purchased from:

Chief Hydrologist

U.S. Geological Survey, WRD

421 National Center

Reston, Virginia 22092

Open-File Services Section Western Distribution Branch U.S. Geological Survey Box 25425, Federal Center Lakewood, Colorado 80225

Telepone: (303) 234-5888 
Abstract . . . . . . . . . . . . . . . . . . . . . 1

Introduction . . . . . . . . . . . . . . . . . . . . . 1

Structure of the identification code . . . . . . . . . . . . . 2

Code assignments . . . . . . . . . . . . . . . . . . . 3

References ........................... . . . 4 4

Identification codes for organizations in ascending order

by organization code . . . . . . . . . . . . . . . . . . 7

Foreign organizations . . . . . . . . . . . . . . . . 7

Federal organizations . . . . . . . . . . . . . . 7

Organizations with national or multistate jurisdiction . . . . . . . . . . . . . . . . . . 9

Non-Federal organizations with State or local jurisdiction . . . . . . . . . . . . . . . . . . 11

Alabama ....................... 11

Alaska ........................ 11

American Samoa ..................... 12

Arizona . . . . . . . . . . . . . . . . . . 12

Arkansas ...................... 13

California ..................... . 13

Colorado ........................ . . . . 24

Connecticut . . . . . . . . . . . . . . . . 25

Delaware ....................... 26

District of Columbia . . . . . . . . . . . . . 26

Florida . . . . . . . . . . . . . . . . 26

Georgia . . . . . . . . . . . . . . . . . 28

Guam . . . . . . . . . . . . . . . . . . 29

Hawail . . . . . . . . . . . . . . . . . 29

Idaho . . . . . . . . . . . . . . . . 30

Illinois ....................... 30

Indiana . . . . . . . . . . . . . . . . . 31

Iowa . . . . . . . . . . . . . . . . 31

Kansas . . . . . . . . . . . . . . . . . 32

Kentucky ....................... 33

Louisiana .................... 33

Maine...................... 34

Maryland ........................ 34

Massachusetts.................. 35 
Milchigan ................... 36

Minnesota . . . . . . . . . . . . . . . 36

Mississippi . . . . . . . . . . . . . . 37

Missouri . . . . . . . . . . . . . . . 38

Montana ..................... 39

Nebraska ...................... 39

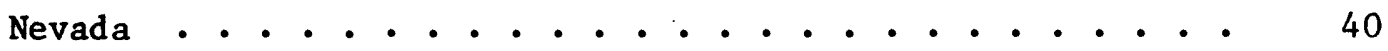

New Hampshire . . . . . . . . . . . . . . . 41

New Jersey . . . . . . . . . . . . . . . . . 41

New Mexico .................... . . . 43

New York .. . . . . . . . . . . . . . 43

North Carolina ................... 44

North Dakota .................... 45

Ohio ....................... 45

Oklahoma .................... . . . 4 46

Oregon ......................... 47

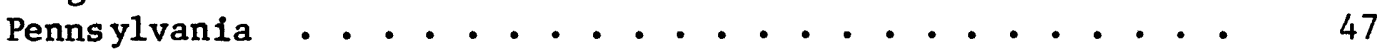

Puerto Rico... . . . . . . . . . . . . . 48

Rhode Island ....................... 49

South Carolina ..................... . 49

South Dakota . . . . . . . . . . . . . . . 52

Tennessee . . . . . . . . . . . . . . . . 53

Texas .......................... 53

Utah . . . . . . . . . . . . . . . . . 55

Vermont . . . . . . . . . . . . . . . 56

Virgin Islands (U.S.) . . . . . . . . . . . . . . 56

Virginia ......................... 56

Wa shington ..................... 57

West Virginia...................... . 59

Wisconsin . . . . . . . . . . . . . . . 59

Wyoming ....................... 60

Identification codes for organizations in alphabetical order

by organization name . . . . . . . . . . . . . . . . 61

Foreign organizations . . . . . . . . . . . . . . 61

Federal organizations . . . . . . . . . . . . . . . 61

Organizations with national or multistate

jurisdiction . . . . . . . . . . . . . . . . 63

Non-Federal organizations with State or local

jurisdiction .. . . . . . . . . . . . . . 65

Alabama . . . . . . . . . . . . . . . . 65

Alaska ........................ 65

American Samoa ................... 66 
Arizona . . . . . . . . . . . . . . . . . . . . 66

Arkansas . . . . . . . . . . . . . . . . . . . 67

California . . . . . . . . . . . . . . . . . . 67

Colorado . . . . . . . . . . . . . . . . . . . 78

Connecticut . . . . . . . . . . . . . . . . . . 79

Delaware . . . . . . . . . . . . . . . . . . . 80

District of Columbia . . . . . . . . . . . . . . . . 80

Florida . . . . . . . . . . . . . . . . . . . 80

Georgia . . . . . . . . . . . . . . . . . . . 82

Guam . . . . . . . . . . . . . . . . . . . 83

Hawail . . . . . . . . . . . . . . . . . . . 83

Idaho . . . . . . . . . . . . . . . . . . . . 84

Illinois . . . . . . . . . . . . . . . . . . 84

Indiana . . . . . . . . . . . . . . . . . . . . 85

Iowa . . . . . . . . . . . . . . . . . . . . 85

Kansas . . . . . . . . . . . . . . . . . . . 86

Kentucky . . . . . . . . . . . . . . . . . . . 87

Louisiana . . . . . . . . . . . . . . . . . . . 87

Maine . . . . . . . . . . . . . . . . . . . 88

Maryl and . . . . . . . . . . . . . . . . . . . 88

Massachusetts . . . . . . . . . . . . . . . . . . 89

Michigan . . . . . . . . . . . . . . . . . . . 90

Minnesota . . . . . . . . . . . . . . . . . . . . 90

Mississippi . . . . . . . . . . . . . . . . . . . . 91

Missouri . . . . . . . . . . . . . . . . . . . . 92

Montana . . . . . . . . . . . . . . . . . . . . . 93

Nebraska . . . . . . . . . . . . . . . . . . . . 93

Nevada . . . . . . . . . . . . . . . . . . . . . 99

New Hampshire • . . . . . . . . . . . . . . . . . 95

New Jersey • • • • • • • • • • • • • • • • • • • . . 95

New Mexico . . . . . . . . . . . . . . . . . . . . 97

New York . . . . . . . . . . . . . . . . . . . . 97

North Carolina . . . . . . . . . . . . . . . . . 98

North Dakota . . . . . . . . . . . . . . . . . . . 99

Ohio . . . . . . . . . . . . . . . . . . . . . . . 99

Oklahoma . . . . . . . . . . . . . . . . . . . . . . . 100

Oregon . . . . . . . . . . . . . . . . . . . . . . 101

Pennsylvania . . . . . . . . . . . . . . . . . . . 101

Puerto Rico . . . . . . . . . . . . . . . . . . . . 102

Rhode Island . . . . . . . . . . . . . . . . . . . 103

South Carolina . . . . . . . . . . . . . . . . . 103

South Dakota . . . . . . . . . . . . . . . . . . 106

Tennessee . . . . . . . . . . . . . . . . . . . 107

Texas . . . . . . . . . . . . . . . . . . . . . 107

Utah . . . . . . . . . . . . . . . . . . . . . 109

Vermont . . . • . . . . . . . . . . . . . . . . . 110

Virgin Islands (U.S.) . . . . . . . . . . . . . . . . 110 
Virginia ..................... 110

Washington . . . . . . . . . . . . . . . 111

West Virginia . . . . . . . . . . . . . . . 113

Wisconsin . . . . . . . . . . . . . . . 113

Wyoming . . . . . . . . . . . . . . . . . 114

\section{ILLUSTRATION}

Page

Figure 1. Agency Code Assignment Request Form . . . . . . . . . . 5 


\section{IDENTIFICATION CODES FOR ORGANIZATIONS LISTED IN COMPUTER IZED DATA SYSTEMS OF THE U.S. GEOLOGICAL SURVEY}

By

Melvin D. Edwards and Beverly M. Josefson

\section{ABSTRACT}

This report contains codes for the identification of public and private organizations listed in computerized data systems. These codes are used by the U.S. Geological Survey's National Water Data Exchange (NAWDEX), National Water Data Storage and Retrieval System (WATSTORE), National Cartographic Information Center (NCIC), and Office of Water Data Coordination (OWDC). The format structure of the codes is discussed and instructions are given for requesting new codes.

\section{INTRODUC TION}

In collaboration with the Office of Water Data Coordination (OWDC), the National Water Data Exchange (NAWDEX) gathers information on Federal, State, and private organizations that are sources of water data and indexes the water data available from many of these organizations. In addition, the U.S. Geological Survey's National Water Data Storage and Retrieval System (WATSTORE) provides facilities for the storage in its files of water data collected by other organizations. These data are also indexed by NAWDEX. A standard coding system is used to uniquely identify each organization participating in these programs.

The National Cartographic Information Center (NCIC) of the U.S. Geological Survey gathers information on the availability and location of cartographic data available from a large number of Federal, State, and private organizations. The enclosed identification codes are also used by NCIC for the identification of non-Federal organizations participating in its program. NCIC uses codes established by the Office of Management and Budget for the identification of Federal organizations.

The Office of Water Data Coordination (OWDC) is responsible for the coordination of water-data acquisition activities of all Federal agencies, as specified by Circular A-67 issued by the office of Management and Budget in August 1964. Many non-Federal organizations also participate in the coordination activities. OWDC uses the enclosed codes for the identification of all organizations participating in its activities, including the Catalog of Information on Water Data. 


\section{STRUCTURE OF THE IDENTIFICATION CODE}

The identification code may contain a minimum of three characters and a maximum of five characters. Codes have been assigned in the following formats.

A. USaaa - This format is used to identify Federal organizations. The first two characters are always the letters "US". The "aaa" is replaced by one to three characters which provide a meaningful abbreviation of the organization name. For example, USGS is the identification code for the U.S. Geological Survey.

B. aaaaa - This format is used to identify organizations having jurisdiction at the international, national, or multiState level. This includes organizations administering Federa1-State compacts, State-to-State compacts and private organizations. From three to five characters are used which provide a meaningful abbreviation of the organization name. This format requires alphabetic characters only and does not allow the characters "US" in the first two positions of the code or the character " $X$ " in the third position of the code. For example, $S R B C$ is the identification code for the Susquehanna River Basin Commission.

C. ss000 - This format is used to identify non-Federal organizations having jurisdiction within a single State boundary. This includes State, county, municipal, private, university, and other local organizations. In this format, the "ss" is replaced by the alphabetic State code identifying the State or outlying area of the United States in which the organization is located. Alphabetic codes in Federal Information Processing Standards Publication Number 5-1 entitled "States and Outlying Areas of the United States" dated June 15, 1970, are used in these positions. The characters "000" are replaced by either a numeric sequence number or a combination of alphabetic and numeric characters which are arbitrarily assigned to uniquely identify each organization. This code format must always be five characters in length. The letter " $X$ " cannot be used in the third character position of the code, and the fifth position of the code must always contain a numeric character. For example, CA001, is the identification code for the California Department of Water Resources.

D. ccXOO - This format is used to identify foreign organizations. The characters "cc" are replaced by the code for the country in which the organization is located. The code is taken from. the Federal Information Processing Standard Publication 
Number 10-2 entitled "Countries, Dependencies, and Areas of Special Sovereignty" dated November 1976. The third position of this code is always the letter " $\mathrm{X}$ ". The characters "00" are replaced by either a numeric sequence number, a combination of alphabetic and numeric characters, or a combination of alphabetic characters which are arbitrarily assigned to identify each organization. For example, CAXO1 is the organization code for the Water Resources Branch of the Canadian Inland Waters Directorate.

\section{CODE ASSIGNMENTS}

A11 organizational codes used by NAWDEX, WATSTORE, and OWDC, and nonFederal organizational codes used by NCIC, are assigned by the NAWDEX Program Office which is located at the Geological Survey's National Center in Reston, Virginia. If additional codes are needed, they may be requested using the form shown in figure 1. The information required in the upper part of the form is mandatory and pertains to the organization for which the code is requested. All items in this part of the form must be completed. The center part of the form is to be completed if the organizational code is requested by an individual from another organization or if the code is to be returned to an individual whose address is different from that given on the upper part of the form. The lower part of the form is for use by the NAWDEX Program office only. The enclosed form may be duplicated or small quantities of the form may be requested from the NAWDEX Program of fice.

Information on organizational codes or requests for organizational code assignments should be directed to:

Program Office

National Water Data Exchange

U.S. Geological Survey

421 National Center

12201 Sunrise Valley Drive

Reston, VA 22092

Telephone: (703) 860-6031,

FTS 928-6031 


\section{REFERENCES}

U.S. Department of Commerce, National Bureau of Standards, 1970, States and outlying areas of the United States: Federal Information Processing Standards Publication 5-1, 4 p.

U.S. Department of Commerce, National Bureau of Standards, 1976, Countries, dependencies, and areas of special sovereignty: Federal Information Processing Standards Publication 10-2, 25 p. 
Form 9-1924

(Nov. 1975)

\section{U.S. DEPARTMENT OF THE INTERIOR \\ CEOLOGICAL SURVEY \\ nawdex \\ Agency Code Assigment Request}

Request date:

Agency code request for (Please provide complete information):

NAME AND/OR TITLE OF

RESPONSIBLE INDIVIDUAL:

ORGANIZATION NAME :

ADDRESS :

$$
\text { Street }
$$

City

State

Zip code

TELEPHONE NO:

TYPE OF REQUEST: New Change

ORGANIZATION TYPE (Check one): Federal

State

County Municipal

Other local University Private

Other (Specify)

REMARKS :

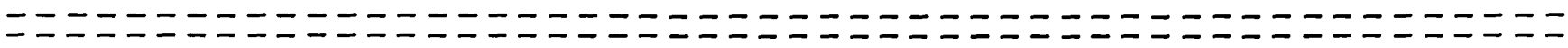
Agency code requested by (Complete only if different than above):

NAME :

ORGANIZATION

ADDRESS :

Street

City

State

Zip code

TELEPHONE NO :

For NAWDEX use only :

REGION CODE :

STATE CODE :

OLD RECORD NO :

NAWDEX AGENCY CODE ASSIGNED:

DATE CODE ASSIGNED:

$\therefore$.COUNT NO:

Approved and logged by:

MAIL REQUEST TO:

National Water Data Exchange

U.S. Geological Survey

421 National Center

12201 Sunrise Valley Drive

Reston, Virginia 22092

Figure 1.--Agency code assignment request form. 
IDENTIFICATION CODES FOR ORGANIZATIONS IN ASCENDING ORDER BY ORGANIZATION CODE

ORGANI -

ZATION

CODE

$$
\text { ORGANIZATION NAME }
$$

FOREIGN ORGANI ZATIONS

FEDERAL ORGANIZATIONS

USAF - AIR FORCE

USAHS - ARMY HEALTH SERVICE

USAPA - ALASKA POWER ADMINISTRATION

USARS - AGRICULTURAL RESEARCH SERVICE 


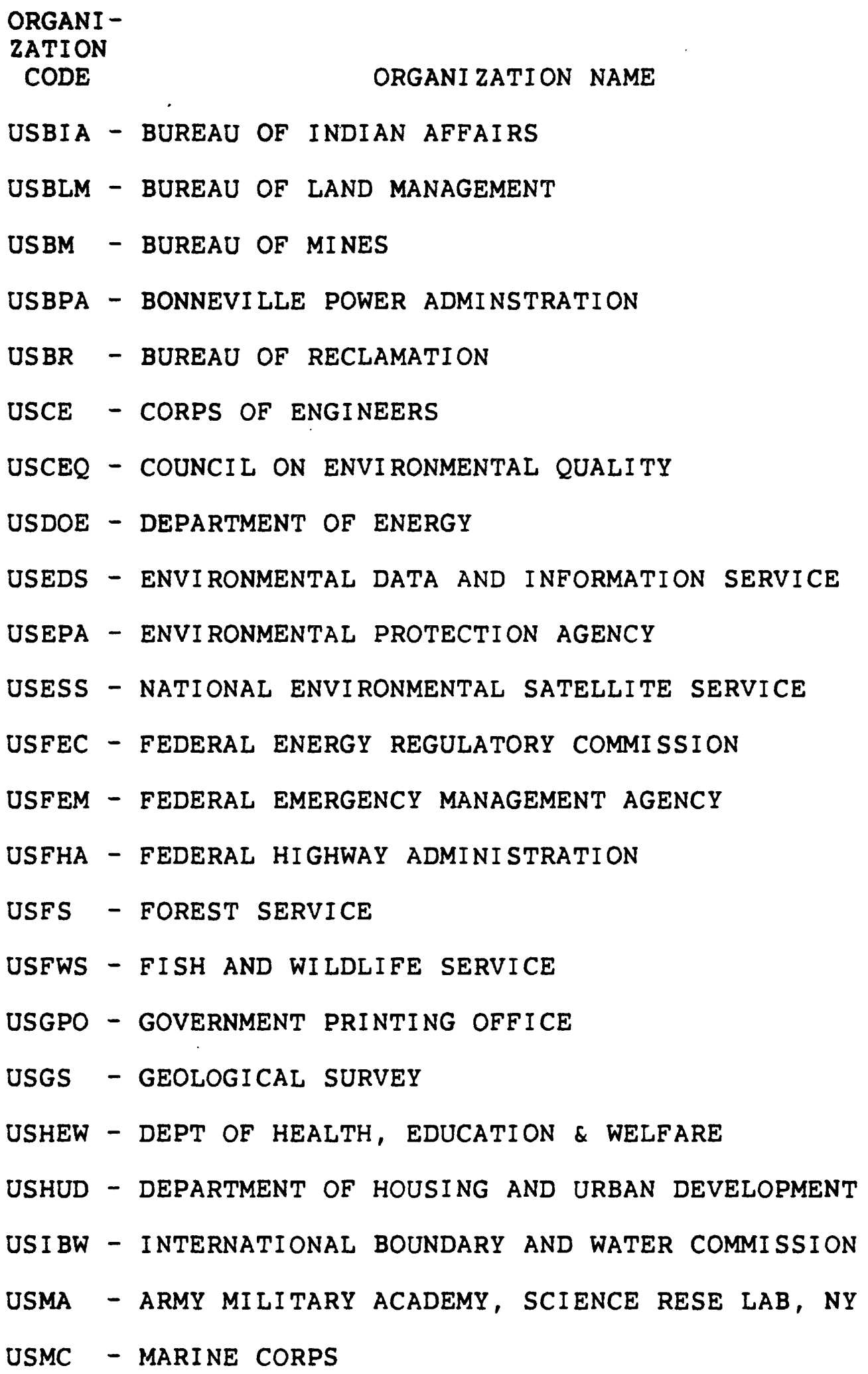


USMFS - NATIONAL MARINE FISHERIES SERVICE

USN - NAVY DEPARTMENT

USNFE - NAVAL FACILITIES ENGINEERING COMMAND

USNOA - NATIONAL OCEANIC AND ATMOSPHERIC ADMINISTRATION

USNOS - NATIONAL OCEAN SURVEY

USNPS - NATIONAL PARK SERVICE

USNRC - NUCLEAR REGULATORY COMMISSION

USNTS - NATIONAL TECHNICAL INFORMATION SERVICE

USNWS - NATIONAL WEATHER SERVICE

USOSM - OFFICE OF SURFACE MINING RECLAM \& ENFORCEMENT

USPCC - PANAMA CANAL COMMISSION

USRDC - RENO FEDERAL DISTRICT COURT (NV)

USSCS - SOIL CONSERVATION SERVICE

USSPA - SOUTHEASTERN POWER ADMINISTRATION

USSWP - SOUTHWESTERN POWER ADMINISTRATION

USTVA - TENNESSEE VALLEY AUTHORI TY

USWRC - WATER RESOURCES COUNCIL

ORGANIZATIONS WITH NATIONAL OR MULTISTATE JURISDICTION

BCC - BOISE CASCADE TIMBER COMPANY

$\mathrm{CHMH}-\mathrm{CH} 2 \mathrm{M} / \mathrm{HILL}$

DMI - DAMES \& MOORE, INC

DRBC - DELAWARE RIVER BASIN COMMISSION 


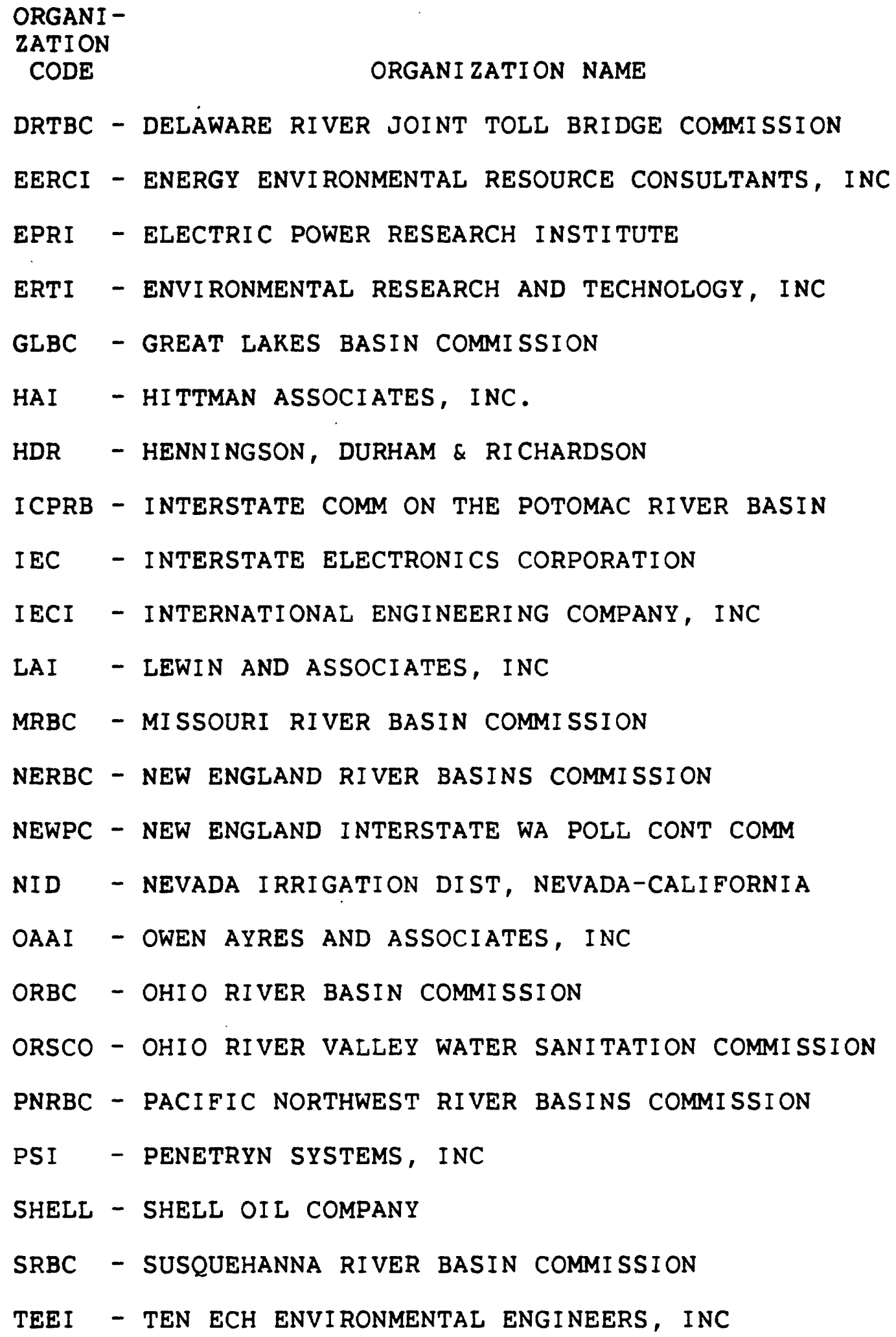


ORGANI -

ZATION

CODE

ORGANI ZATION NAME

UCRBC - UPPER COLORADO RIVER COMMISSION

UMRBC - UPPER MISSISSIPPI RIVER BASIN COMMISSION

WETI - WATER ENGINEERING AND TECHNOLOGY, INC

WMPC - WISCONSIN ELECTRIC POWER COMPANY

NON-FEDERAL ORGANIZATIONS WITH STATE OR LOCAL JURISDICTION

\section{ALABAMA}

ALO0I - GEOLOGICAL SURVEY OF ALABAMA, AL

AL002 - ALABAMA WATER IMPROVEMENT COMMISSION, AL

AL003 - AUBURN UNIV WATER RESOURCES RESEARCH INST, AL

AL004 - ATLANTIC AERIAL SURVEYS, INC, AL

AL005 - WEST ALABAMA PLANNING \& LAND DEVEL COUNCIL, AL

AL006 - ALABAMA STATE HIGHWAY DEPARTMENT, AL

AL007 - ALABAMA DEPARTMENT OF PUBLIC HEALTH, AL

\section{ALASKA}

AKOOI - ALASKA DEPT OF TRANS \& PUBLIC FACILITIES, AK AKOO2 - ALASKA DEPT OF ENVIRONMENTAL CONSERVATION, AK AKO03 - CHUGACH ELECTRIC ASSOCIATION, AK AK004 - ALASKA DEPT OF NATURAL RESOURCES, AK AKO05 - NORTH PACIFIC AERIAL SURVEYS, INC, AK AK006 - STATE OF ALASKA, PIPELINE COORDINATORS OFFICE,AK 
AK007 - AIR PHOTO TECH; INC, AK

AK008 - ALASKA DEPARTMENT OF FISH AND GAME, AK

AKO09 - UNIVERSITY OF ALASKA, AK

\section{AMERICAN SAMOA}

AQOOI - DEPT OF PUBLIC WORKS, GOVT OF AMERICAN SAMOA

ARI ZONA

AZOOI - ARIZONA UNIV, WATER RESOURCES RESE CENTER, AZ

AZ002 - ROOSEVELT IRRIGATION DISTRICT, AZ

AZ003 - ARI ZONA GAME AND FISH DEPARTMENT, AZ

AZ004 - MARICOPA COUNTY MUN WATER CONS DISTRICT \#I, AZ

AZ005 - GILA WATER COMMISSIONER, AZ

AZ006 - SALT RIVER VALLEY WATER USERS ASSOC, AZ

AZ007 - ARIZONA DEPARTMENT OF HEALTH, AZ

AZ008 - UNIV OF ARIZ, COLLEGE-BUSINESS \& PUBLIC ADMIN,AZ

AZ009 - ARI ZONA RESOURCES INFORMATION SYSTEM, AZ

AZOIO - COOPER AERIAL SURVEY COMPANY, AZ

AZOII - SALT RIVER PROJECT, AZ

AZO12 - TOUPS CORPORATION, AZ

AZOI3 - CITY OF TUCSON WATER AND SEWER DEPARTMENT, AZ

AZOI4 - ARIZONA DEPT OF WATER RESOURCES, AZ 
AZ015 - MOTOROLA AERIAL REMOTE SENSING, INC, AZ AZ016 - FAIRCHILD NATIONAL, INC, AZ AZO17 - ARIZONA DEPARTMENT OF TRANSPORTATION, AZ AZ018 - LANDIS AERIAL SURVEYS, AZ AZ019 - AIROGRAPHICS CORPORATION, AZ AZ020 - KENNY AERIAL MAPPING, INC, AZ AZ021 - ARIZONA STATE UNIVERSITY, AZ

ARKANSAS

AROOI - ARKANSAS DEPT OF HEALTH, BUR OF ENVIR ENG, AR AROD2 - ARKANSAS GAME AND FISH COMMISSION, AR AR003 - ARKANSAS DEPT-POLLUTION CONTROL AND ECOLOGY, AR AROO4 - ARKANSAS GEOLOGICAL COMMISSION, AR AROO5 - ARKANSAS HIGHWAY DEPARTMENT, AR AR006 - UNIVERSITY OF ARKANSAS, AR AR007 - ARKANSAS POWER AND LIGHT, AR AR008 - ARKANSAS SOIL \& WATER CONSERVATION COMM, AR

\section{CALIFORNIA}

CAOOI - CALIFORNIA DEPARTMENT OF WATER RESOURCES, CA CA002 - SAN-LO AERIAL SURVEYS INC, CA CA003 - CALIFORNIA DEPARTMENT OF FISH \& GAME, CA 
CA004 - CALIF DEPT TRANS, DIV-HIGHWAYS, PHOTOGRAM BR,CA

CA005 - LOS ANGELES COUNTY FLOOD CONTROL DISTRICT, CA

CA006 - ALAMEDA COUNTY WATER DISTRICT, CA

CA007 - WATER RESO DIV, SACRAMENTO DEPT OF PUB WKS, CA

CA008 - WHITEWATER MUTUAL WATER COMPANY, CA

CA009 - CALIFORNIA STATE WATER RESOURCES CONTROL BRD, CA

CAOIO - COUNTY OF LOS ANGELES, SURVEY DIVISION, CA

CAOI2 - PACIFIC AERIAL SURVEYS, CA

CAOI3 - SIERRA ENVIRONMENTAL MONITORING, CA

CAOI4 - AERIAL MAP INDUSTRIES, CA

CA015 - COACHELLA VALLEY COUNTY WATER DISTRICT, CA

CAOI6 - VENTURA COUNTY FLOOD CONTROL DISTRICT, CA

CAOI7 - SAN DIEGO DEPT OF SANIT \& FLOOD CONTROL, CA

CAOI8 - ORANGE COUNTY ENVIR MANAGEMENT AGENCY, CA

CAOI9 - MERCED IRRIGATION DISTRICT, CA

CA020 - TURLOCK IRRIGATION DISTRICT, CA

CA021 - TRI-DAM PROJ, OAK \& SAN JOAQUIN IRR DIST, CA

CA022 - OROVILLE-WYANDOTTE IRRIGATION DISTRICT, CA

CA023 - MOSQUITO IRRIGATION DISTRICT, CA

CAO24 - CENTRAL CALIFORNIA IRRIGATION DISTRICT, CA

C. 225 - EAST BAY MUNICIPAL UTILITY DISTRICT, CA

CA026 - MODESTO IRRIGATION DISTRICT, CA

CA027 - EL NIDO IRRIGATION DISTRICT, CA 
CA028 - MADERA IRRIGATION DISTRICT, CA

CA029 - HETCH HETCHY WATER SUPPLY, SAN FRANCISCO, CA

CA030 - KINGS RIVER WATER ASSOCIATION, CA

CA031 - FRESNO IRRIGATION DISTRICT, CA

CA032 - KAWEAH \& ST JOHNS WATER ASSOCIATION, CA

CA033 - TULARE IRRIGATION DISTRICT, CA

CA034 - DELANO-EARLIMART IRRIGATION DISTRICT, CA

CA035 - CITY OF BAKERSFIELD DEPT OF WATER, CA

CA036 - BUENA VISTA WATER STORAGE DISTRICT, CA

CA037 - TERRA BELLA IRRIGATION DISTRICT, CA

CA038 - SAUSALITO IRRIGATION DISTRICT, CA

CAO39 - MONTEREY CNTY FLOOD CONT \& WATER CONS DIST, CA

CA040 - SAN LUIS OBISPO CNTY FLOOD CONT-WA CONS DIST, CA

CA041 - MONTECITO COUNTY WATER DISTRICT, CA

CA042 - SANTA BARBARA CNTY FLOOD CONT \& WA CONS DIST, CA

CA043 - METROPOLITAN WATER DIST OF SOUTHERN CALIF, CA

CA044 - MARIN MUNICIPAL WATER DISTRICT, CA

CA045 - NORTH MARIN COUNTY WATER DISTRICT, CA

CA046 - SONOMA CNTY FLOOD CONTROL \& WATER CONS DIST, CA

CA047 - ALAMEDA CNTY FLOOD CONTROL \& WATER CONS DIST, CA

CAO48 - SANTA CLARA VALLEY WATER DISTRICT, CA

CA049 - TULE IRRIGATION DISTRICT, CA

CA050 - MONTAGUE WATER CONSERVATION DISTRICT, CA 
CA051 - LOS ANGELES CITY DEPT OF WATER \& POWER, CA

CA052 - DESERT WATER AGENCY, CA

CA053 - ESCONDIDO MUTUAL WATER COMPANY, CA

CA054 - SAN BERNARDINO COUNTY FLOOD CONTROL DISTRICT, CA

CA055 - SAN ANTONIO WATER COMPANY, CA

CA056 - TEMESCAL WATER COMPANY, CA

CA057 - RIVERSIDE CNTY FLOOD CONT \& WATER CONS DIST, CA

CA058 - VENTURA RIVER MUNICIPAL WATER DISTRICT, CA

CA059 - ORANGE COUNTY HEALTH DEPARTMENT, CA

CA060 - UNITED WATER CONSERVATION DISTRICT, CA

CA06I - KINGS RIVER CONSERVATION DISTRICT, CA

CA062 - LA CANADA IRRIGATION DISTRICT, CA

CA063 - CALIF UNIV-DAVIS-DIV OF ENVIR STUDIES, CA

CA064 - LAHONTAN, CA REG WATER QUAL CONT BRD, CA

CA065 - PACIFIC GAS \& ELECTRIC COMPANY, CA

CA066 - SOUTHERN CALIFORNIA EDISON COMPANY, CA

CA067 - PACIFIC POWER \& LIGHT, CA

CA068 - SAN GABRIEL ELECTRIC COMPANY, CA

CA069 - ORANGE COUNTY DEPARTMENT OF EDUCATION, CA

CA070 - SAN DIEGO STATE UNIVERSITY, CA

CA071 - SHELTER ISLAND LOCKHEED OCEANOGRAPHY LAB, CA.

CA072 - CABRILLO MARINE MUSEUM, CA

CA073 - CALIFORNIA INSTITUTE OF TECHNOLOGY, CA 
CA074 - SANTA CATALINA MARINE LABORATORY, CA

CA075 - CALIFORNIA STATE UNIVERSITY AT LONG BEACH, CA

CA076 - CALIFORNIA STATE COLLEGE AT FULLERTON, CA

CA077 - OCCIDENTAL COLLEGE, CA

CA078 - UNIVERSITY OF CALIFORNIA AT SANTA CRUZ, CA

CA079 - UNIVERSITY OF SANTA CLARA, CA

CA080 - DiABLO VALLEY COLLEGE, CA

CA081 - UNIV CALIFORNIA-BERKELEY, RICHMOND FIELD STA, CA

CA082 - MERRITT COLLEGE, CA

CA083 - MARIN COLLEGE, CA

CA084 - BODEGA BAY MARINE LAB, UNIV OF CA (BERKELEY), CA

CA085 - SAN FRANCISCO PORT AUTHORITY, CA

CA086 - CITY OF LONG BEACH HEALTH DEPARTMENT, CA

CA087 - NAPA CNTY FLOOD CONT \& WA CONS DIST, CA

CA088 - SAN DIEGO COUNTY HEALTH DEPARTMENT, CA

CA089 - LOS ANGELES COUNTY HEALTH DEPARTMENT, CA

CA090 - VENTURA COUNTY HEALTH DEPARTMENT, CA

CA091 - SANTA BARBARA COUNTY HEALTH DEPARTMENT, CA

CA092 - SAN LUIS OBISPO COUNTY HEALTH AGENCY, CA

CA093 - MONTEREY COUNTY HEALTH DEPARTMENT, CA

CAC94 - SANTA CRUZ COUNTY HEALTH DEPARTMENT, CA

CA095 - SAN MATEO COUNTY HEALTH DEPARTMENT, CA

CA096 - SAN FRANCISCO CITY/CNTY BUR-ENVIR HEALTH SERV,CA 
ORGANI -

ZATION

CODE

ORGANI ZATION NAME

CA097 - ALAMEDA COUNTY HEALTH DEPARTMENT, CA

CA098 - HUMBOLDT-DEL NORTE CNTY PUBLIC HEALTH DEPT, CA

CA099 - SONOMA COUNTY PUBLIC HEALTH SERVICE, CA

CA100 - S CALIFORNIA COASTAL WATER RESEARCH PROJECT, CA

CA101 - UNIV OF CALIF AT LOS ANGELES, DEPT OF BIOLOGY,CA

CA102 - HOPKINS MARINE STATION LIBRARY, CA

CAIO3 - MOSS LANDING MARINE LABORATORY, CA

CAI04 - UNIV OF CALIF-BERKELEY-SANIT RESE LAB, CA

CA105 - UNIV OF CALIF-BERKELEY-LAWRENCE LIVERMORE LAB,CA

CA106 - HUMBOLDT STATE UNIVERSITY, DEPT OF FISHERIES, CA

CA107 - HUMBOLDT STATE UNIVERSITY, DEPT OF BIOLOGY, CA

CA108 - HUMBOLDT STATE UNIV, DEPT OF OCEANOGRAPHY, CA

CA109 - ALAMEDA COUNTY OFFICE OF EDUCATION, CA

CA1I0 - CARTWRIGHT AERIAL SURVEYS, INC, CA

CA111 - CALIFORNIA DEPARTMENT OF HEALTH, CA

CA112 - N COAST REGION, CA REGIONAL WTR QUAL CONT BRD,CA

CA113 - SF BAY REGION, CA REGIONAL WTR QUAL CONT BRD, CA

CAI14 - CENTRAL COAST REG, CA REG WTR QUAL CONT BRD, CA

CA115 - LOS ANGELES REG, CA REG WTR QUAL CONT BRD, CA

CAII6 - CENTRAL VALLEY REG, CA REG WTR QUAL CONT BRD, CA

CA117 - COL R BASIN REGION, CA REG WTR QUAL CONT BRD, CA

CA118 - SANTA ANA REGION, CA REGIONAL WTR CONT BRD, CA

CA119 - SAN DIEGO REGION, CA REGIONAL WTR CONT BRD, CA 
CA120 - UNIVERSITY OF SOUTHERN CALIFORNIA, CA

CAI21 - SCRIPPS INST OF OCEANOGRAPHY, SHORE TEMP DATA,CA

CA122 - SCRIPPS INSTITUTE OF OCEANOGRAPHY, CA

CA123 - UNIVERSITY OF CALIFORNIA-SANTA BARBARA, CA

CA124 - UNIV CALIF-SANTA BARBARA, DEPT OF BIOLOGY, CA

CA125 - PLACER COUNTY, DEPT OF PUBLIC WORKS, CA

CA126 - GENGE AERIAL SURVEYS, INC, CA

CA127 - TELEDYNE GEOTRONICS, CA

CA128 - AMERICAN AERIAL SURVEYS INC-NORTHERN CALIF, CA

CA129 - PACIFIC MARINE STATION, CA

CAI30 - UNIV CA AGR EXT, FARM \& HOME ADVISORS OFFICE, CA

CA131 - CONTRA COSTA WATER DISTRICT, CA

CA132 - RIVERSIDE COUNTY DEPARTMENT OF PUBLIC HEALTH, CA

CA133 - IMPERIAL COUNTY HEALTH DEPARTMENT, CA

CA134 - SACRAMENTO CNTY DEPT-PUB WKS, WATER RESO DIV, CA

CA135 - COUNTY OF SAN DIEGO, DEPT OF TRANSPORTATION, CA

CA136 - AMERICAN AERIAL SURVEYS INC-SOUTHERN CALIF, CA

CA137 - MC LAREN ENVIRONMENTAL ENGINEERING, CA

CA138 - PACIFIC ENVIRONMENTAL LABORATORY, CA

CA139 - KAISER ENGINEERS, CA

CA140 - KENNEDY ENGINEERING, CA

CAI41 - HYDRO RESEARCH SCIENCE, CA

CA142 - AIR PHOTO COMPANY, INC, CA 
CA143 - METREX MANAGEMENT CORPORATION, CA

CA144 - I K CURTIS SERVICES, INC, CA

CAI45 - VTN CONSOLIDATED, INC, CA

CA146 - LYON ASSOCIATES, INC, AERIAL MAPPING DIV, CA

CAI47 - CITY OF LONG BEACH ENGINEER, CA

CA148 - HORIZON SURVEYS, CA

CAI49 - PACIFIC WESTERN AERIAL SURVEYS, CA

CA150 - TETRA TECH INC, CA

CA151 - SANTA CLARA CITY WATER DEPARTMENT, CA

CA152 - WESTERN AERIAL SURVEY, CA

CA153 - LAWRENCE BERKELEY LABORATORY, CA

CA154 - WHEELER RIDGE-MARICOPA WATER STORAGE DIST, CA

CA155 - NORTH KERN WATER STORAGE DISTRICT, CA

CA156 - TULARE COUNTY DEPT OF PUBLIC WORKS, CA

CA157 - ALPAUGH IRRIGATION DISTRICT, CA

CAI58 - TULARE LAKE DRAINAGE DISTRICT, CA

CA159 - KINGS COUNTY WATER DISTRICT, CA

CA160 - FRESNO COUNTY DEPT OF HEALTH, CA

CAI6I - FRESNO CITY DEPT OF PUBLIC WORKS, CA

CA162 - MERCED COUNTY DEPT OF HEALTH, CA

こAIË3 - SAN JOAQUIN COUNTY DEPT OF PUBLIC WORKS, CA

CA164 - CALIFORNIA WATER LABS, CA

CA165 - BROWN AND CALDWELL, CA 
CAI91 - ERTEC AIRBORNE SYSTEMS, CA

CAI92 - UPDATA PUBLICATIONS, INC, CA

CA193 - CAL AERO PHOTO, CA

CAI94 - AERO-GEODETIC CORPORATION, CA

CA195 - ASIA MAPPING, INC, CA

CA.196 - HOGAN-SCHOCH \& ASSOCIATES, INC, CA

CAI97 - RICHARD B DAVIS COMPANY, CA

CAI98 - AERO TECH SURVEYS, CA

CAI99 - AIR DATA SYSTEMS, CA

CA200 - PICTORIAL CRAFTS, INC, CA

CA201 - NATIONAL AERO MAPPERS, CA

CA202 - BOYLE ENGINEERING CORPORATION, CA

CA203 - KERN COUNTY DEPT OF PUBLIC WORKS, CA

CA208 - CITY OF SAN DIEGO WATER UTILITIES DEPT, CA

CA209 - POWAY MUNICIPAL WATER DISTRICT, CA

CA2II - SOUTH COAST COMPANY WATER DISTRICT, CA

CA212 - SWEETWATER AUTHORITY, CA

CA213 - CITY OF OCEANSIDE WATER \& SEWER DEPT, CA

CA2I4 - LOS ALISOS WATER DISTRICT, CA

CA215 - RAINBOW MUNICIPAL WATER DISTRICT, CA

CA216 - CITY OF ESCONDIDO WATER \& SEWER MAINTENANCE, CA

CA217 - SANTA MARGARITA WATER DISTRICT, CA

CA218 - ORANGE COAST COLLEGE, CA 
CODE ORGANIZATION NAME

CA166 - KERN COUNTY WATER AGENCY, CA

CA167 - VARA SYSTEMS, CA

CA168 - SAN MATEO COUNTY DEPT OF PUBLIC WORKS, CA

CA169 - WESTERN AERIAL PHOTOS, INC, CA

CA170 - SONOMA COUNTY DEPARTMENT OF PLANNING, CA

CAI71 - SAN BENITO CNTY WATER CONS \& FLOOD CONT DIST, CA

CA172 - MENDOCINO COUNTY PUBLIC HEALTH DEPARTMENT, CA

CAI73 - AERIAL FOTOBANK, CA

CA174 - FOSTER FARMS (DELHI), CA

CA175 - STANISLAUS COUNTY DEPT OF ENVIRON RESOURCES, CA

CAI76 - PACIFIC FIBREBOARD, CA

CA177 - SELMA-KINGSBURG-FOWLER SANITATION DISTRICT, CA

CA178 - F M C CORPORATION, CA

CA179 - ARVIN-EDISON WATER STORAGE DISTRICT, CA

CA180 - WILVERT-PENCEK PHOTOGRAPHIC SERVICES, CA

CA181 - KNUTSON AERIAL PHOTOGRAPHY, CA

CA182 - GRENSTED PHOTOGRAPHY, CA

CA183 - WILSON'S PHOTOGRAPHY, CA

CA184 - TOWILL, INC, CA

CA185 - CALIFORNIA STATE UNIVERSITY AT NORTHRIDGE, CA

CA186 - SOQUEL CREEK COUNTY WATER DISTRICT, CA

CA188 - SACRAMENTO MUNICIPAL UTILITY DISTRICT, CA

CA190 - AERO PHOTOGRAPHERS, INC, CA 
CA219 - BIG BEAR MUNICIPAL WATER DISTRICT, CA

CA220 - YUCAIPA VALLEY COUNTY WATER DISTRICT, CA

CA221 - OTAY MUNICIPAL WATER DISTRICT, CA

CA222 - SANTA FE IRRIGATION DISTRICT, CA

CA223 - CABRILLO SAND AND GRAVEL, CA

CA224 - MONTEREY COUNTY DEPT OF PUBLIC WORKS, CA

CA225 - CALIFORNIA DEPT OF PARKS AND RECREATION, CA

CA226 - SAN FRANCISCO WATER DISTRICT, CA

CA227 - CITY OF ST HELENA, CA

CA228 - MT HERMON ASSOCIATION, CA

CA229 - CALISTOGA CITY WATER DEPT, CA

CA230 - SAN JUAN SUBURBAN WATER DISTRICT, CA

CA232 - CALIF UNIV-IRVINE, WATER RESO LAB, CA

CA233 - HIRSCH AND COMPANY, CA

CA234 - LAKESIDE PARK ASSOCIATION, CA

CA235 - CALIFORNIA-AMERICAN WATER COMPANY, CA

CA236 - BIG BASIN WATER COMPANY, CA

CA237 - CITY OF SANTA CRUZ, CA

CA238 - WATSONVILLE, CITY OF, CA

CA239 - SAN LORENZO VALLEY COUNTY WATER DISTRICT, CA

CA240 - LOMPICO COUNTY WATER DISTRICT, CA

CA241 - CALIFORNIA WATER SERVICE COMPANY, CA

CA242 - NORTH COAST COUNTY WATER DISTRICT, CA 


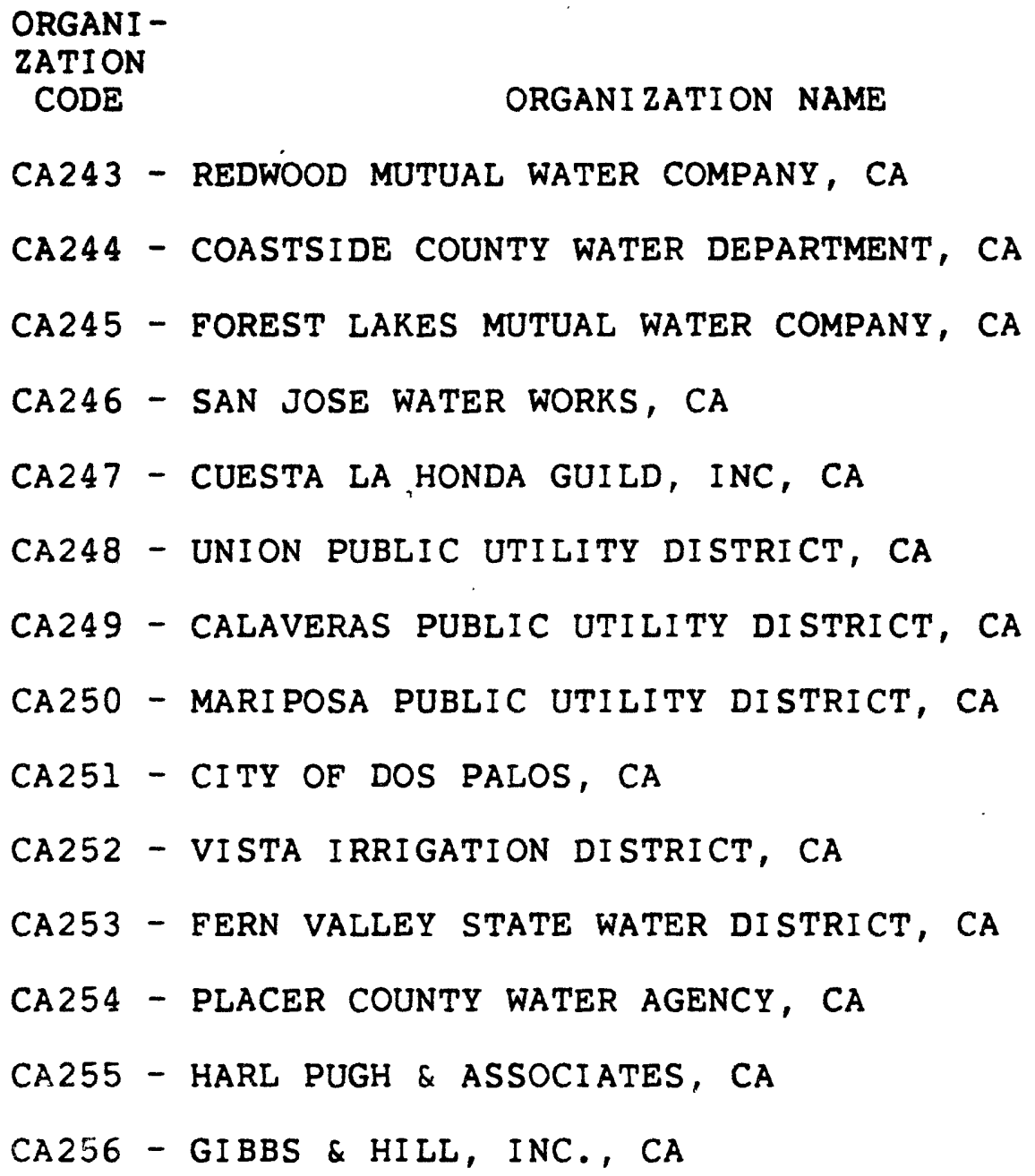

COLORADO

COOD1 - DENVER WATER DEPARTMENT, CO CO002 - COLO DIV OF WATER RESO, OFF OF STATE ENG, CO CO003 - CITY OF COLORADO SPRINGS WATER DIVISION, CO TOOA - BOULDER CITY-COUNTY HEALTH DEPARTMENT, CO CO005 - PUEBLO BOARD OF WATER WORKS, CO CO006 - COLORADO DEPARTMENT OF NATURAL RESOURCES, CO COOO7 - COLORADO STATE HEALTH DEPARTMENT, CO 


\section{ORGANI -}

ZATION

CODE

ORGANIZATION NAME

COO08 - METROPOLITAN DENVER SEWAGE DISPOSAL DIST \#I, CO COOO9 - ROCKY MOUNT ARSENAL, CO

COO10 - COLORADO WATER RESOURCES RESEARCH INST, CO COOII - INTRA SEARCH, INC, CO

CO012 - PIKES PEAK AREA COUNCIL OF GOVERNMENTS, CO COO13 - AGRIC EXPER STA, COLORADO STATE UNIV, CO COOI4 - PUEBLO REGIONAL PLANNING COMMISSION, CO CO015 - OCCIDENTAL OIL SHALE, INC, CO CO016 - WRIGHT WATER ENGINEERS, INC, CO COOI 7 - WESTERN ENGINEERS, INC, CO CO018 - WOODWARD-CLYDE CONSULTANTS, CO CO020 - COLORADO DEPARTMENT OF HIGHWAYS, CO COJ2 1 - W W WHEELER AND ASSOCIATES, INC, CO COO22 - RIO BLANCO OIL SHALE COMPANY, CO CO023 - DENVER REGIONAL COUNCIL OF GOVERNMENTS, CO CO024 - MSM CONSULTANTS, INC, CO

\section{CONNECTICUT}

CTOOI - ENVIR HEALTH SERVICE DIV, CT DEPT OF HEALTH, CT CTO02 - METROPOLITAN (HARTFORD) DISTRICT WATER BUREAU,CT CT003 - CONNECTICUT DEPT OF ENVIRONMENTAL PROTECTION, CT CT004 - BRIDGEPORT HYDRAULIC COMPANY, CT CTO05 - UNIVERSITY OF CONNECTICUT, CT 
CTO07 - CONNECTICUT DEPARTMENT OF TRANSPORTATION, CT CT008 - CITY OF WEST HARTFORD PLANNING DEPT, CT

\section{DELAWARE}

DEO01 - DELAWARE GEOLOGICAL SURVEY, DE DE002 - DELAWARE DEPARTMENT OF NATURAL RESOURCES, DE DE003 - WATER RESOURCES CENTER, UNIV OF DELAWARE, DE DE004 - NEW CASTLE COUNTY WATER RESOURCES AGENCY, DE DE005 - DELAWARE DEPARTMENT OF TRANSPORTATION, DE

\section{DISTRICT OF COLUMBIA}

DCOOI - DIST OF COLUMBIA DEPT OF ENVIR SERVICES, DC DCO03 - WAPORA, INC, DC DCO04 - DISTRICT OF COLUMBIA WATER RESO RESE CENTER, DC DC005 - DISTRICT OF COLUMBIA DEPT OF TRANSPORTATION, DC

\section{FLORIDA}

FLO0I - BUR OF GEOLOGY, FLORIDA DEPT OF NATURAL RESO, FL FLO02 - FLORIDA DEPARTMENT OF TRANSPORTATION, FL FL005 - SOUTH FLORIDA WATER MANAGEMENT DISTRICT, FL FL006 - REEDY CREEK IMPROVEMENT DISTRICT, FL FL007 - SOUTHWEST FLORIDA WATER MANAGEMENT DISTRICT, FL 
ORGANI -

ZATION

CODE

ORGANI ZATION NAME

FLOI2 - WINTER HAVEN BOAT COURSE DISTRICT, FL

FL016 - DADE COUNTY, FL

FL022 - LEE COUNTY, FL

FL030 - VOLUSIA COUNTY, FL

FL039 - CITY OF JACKSONVILLE WATER CONSERVATION, FL

EI043 - CITY OF PENSACOLA, FL

FL05I - FLORIDA DEPT OF ENVIRONMENTAL REGULATION, FL

FL060 - MANATEE COUNTY HEALTH DEPARTMENT, FL

FL069 - SARASOTA COUNTY PUBLIC WORKS, FL

FL083 - NORTHWEST FLORIDA WATER MANAGEMENT DISTRICT, FL

FL084 - SUWANNEE RIVER WATER MANAGEMENT DISTRICT, FL

FL085 - ST JOHNS RIVER WATER MANAGEMENT DISTRICT, FL

LO95 - HILLSBOROUGH COUNTY HEALTH DEPARTMENT, FL

FL096 - HILLSBOROUGH CNTY ENVIR PROTECTION COMM, FL

FL097 - CITY OF LAKE MARY WATER DEPARTMENT, FL

FL098 - SEABURN AND ROBERTSON, INC, FL

FL099 - HUNTER AERIAL SURVEYS, FL

FLI00 - PAN AMERICAN SURVEYS, INC, FL

FLIOI - SOUTH FLORIDA ENVIRON RESEARCH FOUNDATION, FL

FLI03 - CNTR FOR COASTAL ZONE RESE,FL INST OF TECHNOL, FL

FLI04 - REAL ESTATE DATA, INC, FL

FT. १๐- - BROMWELL ENGINEERING, INC, FL

AI :05 - EDWARD E CLARK ENGINEERS-SCIENTISTS, INC, FL 
FLI07 - FLORIDA INSTITUTE OF PHOSPHATE RESEARCH, FL

GEORGI A

GA001 - SAVANNAH DEPARTMENT OF WATER \& SEWAGE, GA

GA002 - THOMASVILLE WATER \& LIGHT DEPARTMENT, GA

GA003 - VALDOSTA WATER \& SEWER DEPARTMENT, GA

GA004 - GAINESVILLE SUPERINTENDENT OF WATER WORKS, GA

GA005 - ROME CITY MANAGER, GA

GA006 - GRIFFIN SUPERINTENDENT OF WATER WORKS, GA

GA007 - MACON-BIBB CNTY WATER \& SEWERAGE AUTHORITY, GA

GA008 - ATLANTA WATER WORKS, GA

GA009 - ENVIR PROT DIV, GEORGIA DEPT OF NATURAL RESO, GA

GAO10 - COLUMBUS WATER WORKS, GA

GAOII - SOUTHWEST GEORGIA PLANNING \& DEVEL COMM, GA

GAOI 2 - GA DEPT NAT RESO, GEOLOGIC \& WA RESO DIV, GA

GA013 - GEORGIA INST OF TECHNOLOGY, ENVIR RESO CNTR, GA

GAOI4 - LOWE ENGINEERS, INC, GA

GA015 - CITY OF SAVANNAH INDUST \& DOMESTIC WA SUPPLY, GA

GAOI6 - GEORGIA DEPARTMENT OF TRANSPORTATION, GA

GA017 - LAW ENGINEERING TESTING COMPANY, GA

GAOI8 - MARINE EXTENSION SERVICE, UNIV OF GEORGIA, GA

GAO19 - MC INTOSH COUNTY OFFICE OF TAX ASSESSOR, GA

GAO20 - CHATHAM CNTY-SAVANNAH METRO PLANNING COMM, GA 
GA021 - CAMDEN COUNTY OFFICE OF TAX ASSESSOR, GA GA022 - BRUNSWICK PULP LAND CO--WOODS DIVISION, GA GA023 - CITY OF ATLANTA DEPT OF PUBLIC WORKS, GA

GUAM

GQ008 - PUBLIC UTILITY AGENCY-WATER DIV, GOVT OF GUAM GQ009 - WATER RESOURCES RESE CENTER, UNIV OF GUAM GQOII - DEPT OF PUBLIC WORKS, GOVT OF GUAM

\section{HAWA I}

HIOOI - BOARD OF WATER SUPPLY, CITY \& CNTY-HONOLULU, HI HIO02 - DEPT OF WATER, COUNTY OF KAUAI, HI HIO03 - BOARD OF WATER SUPPLY, COUNTY OF MAUI, HI HI004 - BOARD OF WATER SUPPLY, COUNTY OF HAWAII, HI HIO05 - HAWAII DEPT OF HAWAIIAN HOME LANDS, HI HI006 - DIV-FISH \& GAME, HI DEPT OF LAND \& NAT RESO, HI HI007 - DIV-WATER \& LAND DEV, HI DEPT-LAND \& NAT RESO,HI HI008 - HAWAII DEPT OF TRANSPORTATION, HI HI009 - R M TOWILL CORPORATION, HI HIOIO - WATER RESOURCES RESE CNTR, UNIV OF HAWAII, HI HTOII - AIR SURVEY HAWAII, HI 
IDAHO

IDOOI - IDAHO DEPARTMENT OF WATER RESOURCES, ID

IDO02 - IDAHO FISH AND GAME DEPARTMENT, ID

IDOO3 - WATER RESOURCES RESEARCH INST, UNIV OF IDAHO, ID

IDOO4 - IDAHO DEPARTMENT OF HEALTH AND WELFARE, ID

IDO05 - IDAHO DEPT OF PUBLIC LANDS--TECH SERV SECT, ID

ID006 - IDAHO REPROGRAPHICS, ID

ID007 - AERIAL MAPPING COMPANY, ID

ID008 - IDAHO DEPT-TRANSPORTATION, DIV OF HIGHWAYS, ID

IDO09 - ADA COUNTY PLANNING ASSOCIATION, ID

IDOIO - IDAHO STATE COLLEGE, ID

IDOII - IDAHO BUREAU OF MINES AND GEOLOGY, ID

IDO12 - WESTERN WAYS, INC, ID

IDOI3 - POTLATCH CORPORATION, TIMBERLANDS DEPT, ID

\section{ILLINOIS}

ILOOI - ILLINOIS DEPARTMENT OF PUBLIC HEALTH, IL

ILO02 - METRO SANITARY DIST OF GREATER CHICAGO, IL

IL003 - ILLINOIS STATE WATER SURVEY, IL

ILOO4 - ILLINOIS DEPT OF TRANSPORTATION, IL

¿005 - DEPAUL UNIVERSITY, IL

ILO06 - STATE OF ILL ENVIRONMENTAL PROTECTION AGENCY, IL 
ORGANI -

ZATION

CODE

ORGANI ZATION NAME

IL007 - WATER RESOURCES CENTER, UNIV OF ILLINOIS, IL

IL008 - ILLINOIS INSTITUTE OF NATURAL RESOURCES, IL

ILOI0 - ILLINOIS NATURAL HISTORY SURVEY, IL

ILOII - SIDWELL COMPANY, IL

IL012 - TECHNICAL PUBLISHING CO--POLIUT ENGINEERING, IL

ILOI3 - NALCO CHEMICAL COMPANY, IL

ILOI4 - RANDOLPH \& ASSOCIATES, INC, IL

ILOI5 - ARGONNE NATIONAL LABORATORY, IL

IL0I6 - CHICAGO AERIAL SURVEY, IL

ILOI7 - SARGENT\& LUNDY ENGINEERS, IL

IL018 - NORTHEASTERN ILLINOIS PLANNING COMMISSION, IL

I NDI ANA

INOOI - IND ST BRD OF HEALTH, DIV STREAM POLL CONT, IN

INO02 - INDIANA DEPARTMENT OF NATURAL RESOURCES, IN

IN004 - INDIANA PLANNING \& DEVEL COM, REG 6, IN

INO05 - INDIANA STATE BOARD OF HEALTH, IN

INO06 - PURDUE UNIVERSITY, IN

IN007 - HOLCOMB RESEARCH INSTITUTE, IN

INO08 - INDIANA STATE HIGHWAY COMMISSION, IN

IOWA

IAOOI - UNIVERSITY HYGIENIC LABORATORY, IA 
IAO02 - LAKESIDE LABORATORY, UNIV OF IOWA, IA

IA003 - DES MOINES WATER WORKS, IA

IAO04 - OTTUMWA WATER WORKS, IA

IAO05 - DEPARTMENT OF CIVIL ENGINEERING, UNIV OF IOWA, IA

IA006 - IOWA DEPT OF PREVENT MEDICINE \& ENVIR HEALTH, IA

IA007 - AGRICULTURAL ENG DEPARTMENT, IOWA STATE UNIV, IA

IA008 - FORT DODGE DEPT OF MUNICIPAL UTILITIES, IA

IA009 - COUNCII BLUFFS WATER WORKS, IA

IA010 - DES MOINES COUNTY DRAINAGE DIST NO 7, IA

IAOII - GREEN BAY LEVEE \& DRAINAGE DIST NO 2, IA

IAOI2 - DEPARTMENT OF CIVIL ENG, IOWA STATE UNIV, IA

IAOI3 - DEPARTMENT OF GEOGRAPHY, UNIV OF IOWA, IA

IAOI4 - IOWA INST OF HYDRAULIC RESEARCH, UNIV OF IOWA, IA

IAOI5 - ENGINEERING RESEARCH INSTITUTE, IA STATE UNIV, IA

IA0I6 - IOWA STATE AGR EXP STATION, IA STATE UNIV, IA

IAOI7 - DEPARTMENT OF EARTH SCIENCE, IOWA STATE UNIV, IA

IAOI8 - IOWA GEOLOGICAL SURVEY, IOWA CITY, IA

IAOI9 - IOWA DEPARTMENT OF ENVIRONMENTAL QUALITY, IA

IAO20 - IOWA DEPARTMENT OF TRANSPORTATION, IA

KANSAS

KSOOI - ENVIR HEALTH SERVICE, KS STATE DEPT OF HEALTH,KS KSOO2 - KANSAS CITY BOARD OF PUBLIC UTILITIES, KS 
KS003 - DIV OF WATER RESO, KANSAS STATE BRD OF AGR, KS KS004 - TOPEKA WATER DEPARTMENT, KS KSO05 - KANSAS FISH \& GAME COMMISSION, KS KSO06 - KANSAS DEPARTMENT OF TRANSPORTATION, KS

\section{KENTUCKY}

KYOOI - KENTUCKY DEPT FOR NAT RESO \& ENVIR PROT, KY KYOO2 - KENTUCKY GEOLOGICAL SURVEY, UNIV OF KENTUCKY, KY KY003 - DIV-SANIT ENG, KY DEPT FOR HUMAN RESOURCES, KY KY004 - LOUISVILLE WATER COMPANY, KY KYO05 - KENTUCKY DEPT OF FISH \& WILDLIFE, KY KYOO7 - WATER RESOURCES LAB, UNIV OF LOUISVILLE, KY KY008 - WATER RESOURCES RESE INST, UNIV OF KENTUCKY, KY KYOO9 - KENTUCKY DEPARTMENT OF TRANSPORTATION, KY KYOIO - PARK AERIAL SURVEYS, KY KYOII - GRW AERIAL SURVEY, INC, KY

LOUI SI ANA

LAO0I - RAPIDES PARISH WATER WORKS DISTRICT NO 3, LA LA002 - LOUISIANA STATE DEPARTMENT OF HEALTH, LA LA003 - HOUMA WATER TREATMENT PLANT, LA LAO04 - JEFFERSON WATER WORKS DISTRICT NO 2, LA 
ORGANI -

ZATION

CODE

ORGANI ZATION NAME

LAO05 - LAFOURCHE WATER WORKS DISTRICT NO 1, LA

LA006 - EAST JEFFERSON WATER WORKS DISTRICT NO 1, LA

LA007 - NEW ORLEANS SEWERAGE \& WATER BOARD, LA

LA008 - BOSSIER CITY WATER PLANT, LA

LAOO9 - MONROE UTILITIES COMM WATER TREATMENT PLANT, LA

LAOIO - DIV-WATER POLL CONT, LA WILDLIFE \& FISH COMM, LA

LAOI1 - SHREVEPORT DEPARTMENT OF WATER \& SEWERAGE, LA

LA012 - CENTER FOR WETLAND RESOURCES (LSU), LA

LA013 - LOUISIANA DEPT OF WILDLIFE AND FISHERIES, LA

LA014 - LOUISIANA DEPT OF TRANSPORT \& DEVELOPMENT, LA

\section{MAINE}

MEO0I - MAINE DEPT OF ENVIRONMENTAL PROTECTION, ME ME002 - MAINE GEOLOGICAL SURVEY, ME

ME003 - MAINE STATE PLANNING OFF, RESO PLANNING DIV, ME

ME004 - MAINE DEPARTMENT OF HUMAN SERVICES, ME

ME005 - MAINE DEPT OF INLAND FISHERIES AND WILDLIFE, ME

ME006 - UNIVERSITY OF MAINE, ME

ME007 - MAINE DEPT OE MARINE RESOURCES, ME

ME008 - MAINE DEPARTMENT OF TRANSPORTATION, ME

MARYLAND

MDO01 - BALTIMORE COUNTY HEALTH DEPARTMENT, MD 
MD002 - BALTIMORE WATER SUPPLY TREATMENT \& PUMP DIV, MD MD003 - MARYLAND STATE DEPT OF NATURAL RESOURCES, MD MDOO4 - MONTGOMERY COUNTY DEPT OF ENVIRONMENTAL PROT, MD MD005 - PRINCE GEORGES COUNTY DEPARTMENT OF HEALTH, MD MDO06 - MARYLAND GEOLOGICAL SURVEY, MD MD008 - BALTIMORE COUNTY OFFICE OF PLANNING \& ZONING, MD MDOO9 - ENVIRO CONTROL, INC, MD MDO10 - MARYLAND DEPARTMENT OF TRANSPORTATION, MD MDO12 - AEROECO, MD MD013 - SIGMA DATA COMPUTING CORPORATION, MD MDOI4 - GENERAL SOFTWARE CORPORATION, MD MDO15 - GREENHORNE AND O'MARA, INC, MD MDO16 - MARYLAND DEPARTMENT OF STATE PLANNING, MD

\section{MASSACHUSETTS}

MAO0I - GIDLEY LABORATORIES, INC, MA MAO02 - WATER RESOURCES RESEARCH CNTR, UNIV OF MASS, MA MAOO3 - MASSACHUSETTS DIV OF WATER POLLUTION CONTROL, MA MAO04 - WILLIAM T LORENZ \& COMPANY, MA MAOO5 - THERMO-ELECTRIC CORPORATION, MA MA006 - MILLIPORE CORPORATION, MA MA007 - BARNSTABLE COUNTY HEALTH DEPARTMENT, MA MA008 - MASSACHUSETTS DEPARTMENT OF PUBLIC WORKS, MA 
MA009 - AVIS AIRMAP, INC, MA

MAOIO - MASSACHUSETTS WATER RESOURCES COMMISSION, MA MAOII - BOSTON REDEVELOPMENT AUTHORITY, MA

\section{CHIGAN}

MIO0I - MICHIGAN DEPARTMENT OF NATURAL RESOURCES, MI MI002 - MICHIGAN STATE UNIVERSITY, MI MIO03 - NATIONAL SANITATION FOUNDATION, MI MI004 - MICHIGAN GRAND RIVER WATERSHED, MI MIO05 - NORTHWEST WATERSHED PROJECT, MI MIO06 - JOHNSON \& ANDERSON, INC, MI MIO07 - ABRAMS AERIAL SURVEY CORPORATION, MI MIO08 - BENDIX AEROSPACE SYSTEMS DIVISION, MI MI009 - CAPITOL AIR SURVEY, MI MIOIO - ENVIRONMENTAL RESEARCH INSTITUTE OF MICHIGAN, MI MIOII - MICH DEPT OF STATE HIGHWAYS\& TRANSPORTATION, MI MI013 - S CENTRAL MICH PLAN \& DEVEL COUNCIL-REG III, MI MIOI4 - SOUTHEAST MICHIGAN COUNCIL OF GOVERNMENTS, MI MIOI5 - STEREO FOTO, INC, MI MIOI6 - WEST MICHIGAN REGIONAL PLANNING COMM, MI

\section{MINNESOTA}

MNOOI - HENNEPIN COUNTY HIGHWAY DEPARTMENT, MN 
ORGANI -

ZATION

CODE

ORGANI ZATION NAME

MNO02 - EVELETH TACONITE COMPANY, MN

MN003 - MINNESOTA DEPT OF NATURAL RESOURCES, MN

MNO04 - OTTER TAIL POWER COMPANY, MN

MNO05 - RAMSEY COUNTY ENVIRONMENTAL SERVICE, MN

MN006 - NORTHERN STATE POWER COMPANY, MN

MN007 - DULUTH WATER, GAS \& SEWAGE TREATMENT DEPT, MN

MN008 - MINNESOTA ORE OPER, USS CORP, MN

MNO09 - BLANDIN PAPER COMPANY, MN

MNOIO - MINNESOTA POWER \& LIGHT COMPANY, MN

MNOII - METROPOLITAN (ST PAUL) WASTE CONTROL COMM, MN

INOI2 - MINNESOTA POLLUTION CONTROL AGENCY, MN

MNO13 - WASHINGTON COUNTY HIGHWAY DEPARTMENT, MN

MDI4 - UNITED POWER ASSOCIATION, MN

ZNOI5 - MARK HURD AERIAL SURVEYS, INC, MN

MNOI6 - MINNESOTA STATE PLANNING AGENCY, MN

MNO17 - WATER RESOURCES RESEARCH CTR, UNIV OF MINN, MN

I.INO18 - MINNESOTA DEPT OF TRANSPORTATION, MN

MNOI9 - MINNESOTA HEALTH DEPARTMENT, MN

MNO20 - MARTINEZ MAPPING AND ENGINEERING, INC, MN

MN021 - METROPOLITAN COUNCIL OF THE TWIN CITIES AREA, MN

MI SSI SSI PPI

MSOOI - CITY OF VICKSBURG WATER TREATMENT PLANT, MS 
MSO02 - CITY OF JACKSON WATER WORKS, MS

MSO03 - PEARL RIVER VALLEY WATER SUPPLY DISTRICT, MS MS004 - CITY OF MERIDIAN WATER \& SEWER DEPARTMENT, MS MS005 - CITY OF COLUMBUS LIGHT \& WATER DEPARTMENT, MS MS006 - DIV OF SANIT ENG, MISS STATE BOARD OF HEALTH, MS MSO07 - MS DEPT NAT RESO-BUR OF POLL CONT, MS MS008 - MISSISSIPPI BUR OF LAND \& WATER RESOURCES, MS MSO09 - PAT HARRISON WATERWAY DISTRICT, MS MSOIO - MISSISSIPPI MARINE RESOURCES COUNCIL, MS MSOII - MISSISSIPPI BUR OF GEOLOGY-DEPT OF NAT RESO, MS MSOI2 - MISSISSIPPI STATE HIGHWAY DEPARTMENT, MS MSO13 - MISSISSIPPI STATE UNIV, MS

\section{SSOURI}

MO0OI - MISSOURI DIVISION OF HEALTH, MO MO002 - UNIVERSITY OF MISSOURI, MO MO003 - METROPOLITAN ST LOUIS SEWER DISTRICT, MO MO004 - LITTLE RIVER DRAINAGE DISTRICT, MO MO005 - MO DIV OF GEOL \& LAND SURVEY, DEPT-NAT RESO, MO MO006 - MISSOURI WATER POLLUTION CONTROL PROGRAM, MO MO007 - UNION ELECTRIC COMPANY, MO MO008 - WATER RESOURCES RESE CNTR, UNIV OF MISSOURI, MO MOOO9 - SURDEX CORPORATION, MO 
MOOIO - MID-AMERICA REGIONAL COUNCIL, MO

MOOII - BURNS AND MC DONNELL, MO

MOOI2 - MISSOURI STATE HIGHWAY DEPARTMENT, MO

MO013 - UNICORN FEASIBILITY, MO

MOOI4 - BLACK \& VEATCH CONSULTING ENGINEERS, MO

MONTANA

MTOOI - MONTANA DEPT OF FISH, WILDLIFE \& PARKS, MT

MTO02 - MONTANA UNIV JOINT WATER RESOURCES RESE CNTR, MT

MTOO3 - MONTANA DEPARTMENT OF HEALTH \& ENVIR SCIENCES,MT

MTOO4 - MONTANA DEPT OF NATURAL RESO \& CONSERVATION, MT

MTOO5 - MONTANA BUREAU OF MINES \& GEOLOGY, MT

MTO06 - WESTECH, INC, MT

MT007 - UNIVERSITY OF MONTANA, MT

MTO08 - MONTANA ENERGY \& MHD RESE \& DEVELOPMENT INST, MT MTO09 - MONTANA DEPARTMENT OF HIGHWAYS, MT

NEBRASKA

NEOOI - NEBRASKA GAME \& PARKS COMMISSION, NE

J002 - WATER POLL CONT DIV, NEBR DEPT OF ENVIR CONT, NE NERO? - OMAHA METROPOLITAN UTILITIES DISTRICT, NE IFO04 - SOIL \& WATER TESTING LAB, UNIV OF NEBRASKA, NE 
NE005 - NEBRASKA DEPARTMENT OF HEALTH, NE

NE006 - NEBRASKA NATURAL RESOURCES COMMISSION, NE

NE007 - WATER RESOURCES CENTER, UNIV OF NEBRASKA, NE

NEOO8 - UNIV OF NEBRASKA, CONSERVATION \& SURVEY DIV, NE NE009 - NEBRASKA DEPARTMENT OF ROADS, NE

NEOIO - NEBRASKA DEPARTMENT OF WATER RESOURCES, NE NE01I - HOSKINS-WESTERN-SONDEREGGER, INC, NE

\section{NEVADA}

NVOOI - NEVADA DIVISION OF ENVIRONMENTAL PROTECTION, NV NVO02 - WALKER RIVER IRRIGATION DISTRICT, NV NV003 - DIV-WATER RESO, NEVADA DEPT-CONS \& NAT RESO, NV NVOO4 - ROUNDHILL GENERAL IMPROVEMENT DISTRICT, NV NV005 - DIV OF RENEWABLE NAT RESO, UNIV OF NEVADA, NV NV006 - NEVADA STATE BUREAU OF ENVIRONMENTAL HEALTH, NV NV007 - NEVADA BUREAU OF MINES \& GEOLOGY, NV NV008 - NEVADA STATE HIGHWAY DEPARTMENT, NV NV009 - NEVADA DEPARTMENT OF FISH \& GAME, NV NVOIO - NEVADA DIVISION OF FORESTRY, NV NVOI1 - NEVADA DIVISION OF PARKS, NV NV012 - NEVADA CONSUMER HEALTH PROTECTION SERVICE, NV NV013 - DESERT RESEARCH INSTITUTE, UNIV OF NEVADA, NV NVO14 - COLLEGE OF AGRICULTURE, UNIV OF NEVADA, NV 
NVOI5 - CLARK COUNTY HEALTH DISTRICT, NV NV016 - WASHOE COUNTY DISTRICT HEALTH DEPARTMENT, NV NV017 - LAS VEGAS VALLEY WATER DISTRICT, NV NV018 - SIERRA PACIFIC POWER COMPANY, NV NVO20 - WASHOE COUNCIL OF GOVERNMENTS, NV NVO21 - CARSON RIVER BASIN COUNCIL OF GOVERNMENTS, NV NV023 - WALTERS ENGINEERING, INC, NV NVO25 - RENO-SPARKS JOINT WASTE WAT POLLUT CONT PLANT,NV NV026 - CARSON CITY DEPT OF PUBLIC WORKS, NV NVO27 - UPSON-DONDERO STUDIOS, NV NV028 - KAMINSKI STUDIOS LTD, NV NVO29 - GREAT BASIN AERIAL SURVEYS, NV

\section{NEW HAMPSHIRE}

NHOO1 - WATER RESO RESE CNTR, UNIV OF NEW HAMPSHIRE, NH NHOO2 - NEW HAMPSHIRE WAT SUPPLY \& POLLUT CONTROL COM,NH NHOO3 - NEW HAMPSHIRE DEPT OF PUB WKS AND HIGHWAYS, NH NHOO4 - BCI GEONETICS, INC, NH

NEW JERSEY

NJOOI - PASSAIC VALLEY WATER COMMISSION, NJ NJ002 - DIV-WATER RESO, NJ DEPT OF ENVIR PROTECTION, NJ 


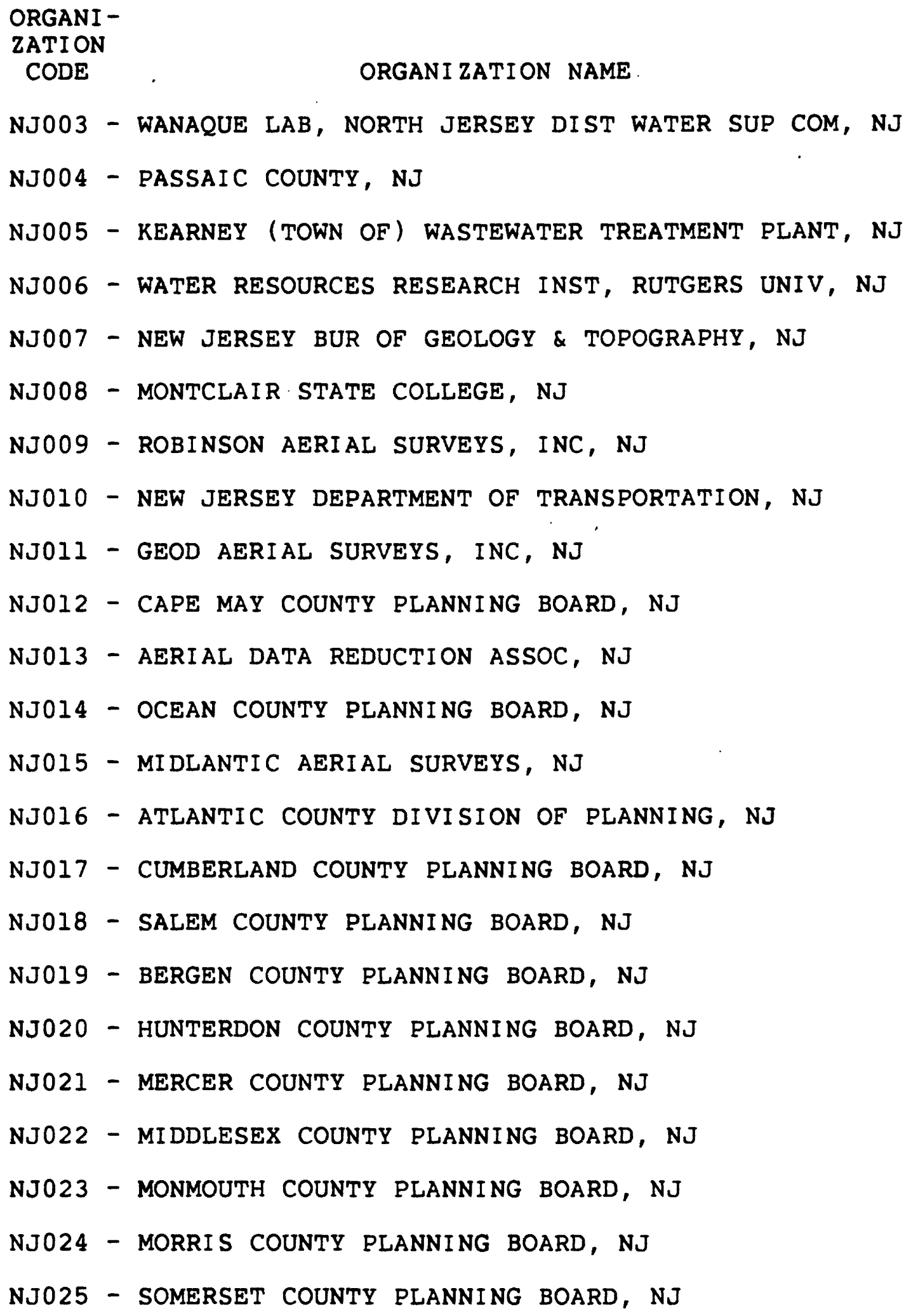


NJO26 - UNION COUNTY DIV OF PLANNING \& DEVEL, NJ

NEW MEXICO

NMOOI - NEW MEXICO STATE ENGINEERS OFFICE, NM NM002 - N MEX STATE HEALTH \& ENVIRONMENT DEPT, NM NMOO3 - NEW MEXICO INST OF MINING AND TECHNOLOGY, NM NMO04 - NEW MEXICO STATE UNIVERSITY, NM NMO05 - LOS ALAMOS SCIENTIFIC LABORATORY, NM NMO06 - THOMAS R MANN AND ASSOCIATES, NM NM007 - NEW MEXICO STATE HIGHWAY DEPARTMENT, NM

\section{NEW YORK}

NYOOI - NEW YORK STATE DEPT OF ENVIRONMENTAL CONS, NY NY002 - DIV-SANIT \& WATER SUP, NASSAU CNTY PUB SUP, NY NY003 - THE CARBORUNDUM COMPANY, NY NY004 - TRI-STATE REGIONAL PLANNING COMMISSION, NY NY005 - AEROGRAPHICS, INC, NY NY006 - CITY OF ROCHESTER WATER TREATMENT PLANT, NY NYOO7 - NEW YORK CITY DEPT OF ENVIR PROTECTION, NY NY008 - WESTCHESTER COUNTY DEPT OF PUBLIC WORKS, NY NYOO9 - MONROE COUNTY WATER AUTHORITY, NY NYOIO - ONONDAGA COUNTY DEPT OF DRAINAGE \& SANIT, NY 
NYO11 - RENNSELAER POLYTECHNIC INSTITUTE, NY NY012 - NY STATE UNIV AT BUFFALO, GREAT LAKES LAB, NY NY013 - CORNELL UNIVERSITY, NY NYO14 - BROOKHAVEN NATIONAL LABORATORY, NY NY015 - LOCKWOOD, KESSLER \& BARTLETT, INC, NY NY016 - NYC BUR OF WATER POLLUTION CONTROL, NY NYOI7 - NEW YORK PUBLIC INTEREST RESEARCH GROUP, NY NY018 - AERO SERVICE CORPORATION, NY NYO19 - SANBORN MAP COMPANY, INC, NY NY020 - GEO MAP, INC, NY NY02I - NY STATE EDUCATION DEPARTMENT, NY NYO22 - NEW YORK STATE DEPT OF HEALTH, NY NY025 - DONALD A THOMAS, LAND SURVEYOR (GENESEO), NY NY028 - NEW YORK STATE ELECTRIC \& GAS CORPORATION, NY NY029 - EL COMP DATA SYSTEMS, INC, NY NY030 - O'BRIEN AND GERE ENGINEERS, INC, NY NY031 - SCHENECTADY COUNTY PLANNING DEPT, NY NY032 - CITY OF ALBANY PLANNING DEPT, NY

NORTH CAROLINA

NCOO3 - WATER SUPPLY BR, SES, DHS, NC DEPT HUMAN RESO,NC NCOO4 - NC DEPT-NAT RESO \& COMMUNITY DEVELOPMENT, NC NCOO5 - DUKE UNIV, SCHOOL OF ENG, DEPT OF CIVIL ENG, NC 
NC006 - SOUTHERN WTR RESO SCIENTIFIC INFO CNTR, NCU, NC NC007 - PIEDMONT AERIAL SURVEYS, INC, NC NCOO8 - CAROLINA POWER \& LIGHT COMPANY, NC NCO09 - NC UNIV, DEPT OF ENV SCIENCE \& ENG, NC NCO10 - CHARLES T MAIN, INC, NC NCOII - NORTH CAROLINA DEPT OF TRANSPORTATION, NC NC012 - YONTS AND ASSOCIATES, NC NCO13 - CITY OF GREENSBORO DEPT OF PUBLIC WORKS, NC

NORTH DAKOTA

NDOOI - NORTH DAKOTA GAME \& FISH DEPARTMENT, ND NDO02 - NORTH DAKOTA STATE DEPARTMENT OF HEALTH, ND ND003 - MINOT CITY WATER TREATMENT PLANT, ND ND004 - CITY OF BISMARCK WATER DEPARTMENT, ND ND005 - CITY OF DICKINSON WATER TREATMENT PLANT, ND ND006 - GRAND FORKS WATER TREATMENT PLANT, ND ND007 - NORTH DAKOTA GEOLOGICAL SURVEY, ND NDOO9 - NORTH DAKOTA STATE WATER COMMISSION, ND NDO10 - WATER RESOURCES RESEARCH INST, ND STATE UNIV, ND NDO11 - NORTH DAKOTA STATE HIGHWAY DEPARTMENT, ND 
OHIO

OHOO2 - MIAMI CONSERVANCY DISTRICT, OH

OH004 - OHIO ENVIRONMENTAL PROTECTION AGENCY, OH OH005 - CITY OF CLEVELAND QUALITY WATER DEPARTMENT, OH OHOO6 - DIV OF HIGHWAYS, QHIO DEPT OF TRANSPORTATION, OH OHO07 - WATER RESOURCES CENTER, OHIO STATE UNIV, OH OHO08 - GEAUGA COUNTY OFFICE OF SANITARY ENGINEER, OH OH009 - AERIAL ENG SECT, OHIO DEPT-TRANSPORTATION, OH OHO10 - OHIO STATE UNIVEPSTTY, DEPT OF AGRONOMY, OH OHO11 - OHIO AGRICULTURAL RESE \& DEVELOPMENT CENTER, OH OHO12 - GRAPHCO, OH OHO13 - HEIDELBERG COLLEGE, OH OHO14 - OHIO STATE UNIV CENTER FOR LAKES, OH OH015 - OHIO DEPT OF NATURAL RESOURCES, OH OH016 - COLE-LAYER-TRUMBLE, INC, OH OH017 - KUCERA \& ASSOCIATES, OH OH018 - CITY OF CINCINNATI PLANNING DEPT, OH OHO19 - CUYAHOGA COUNTY PLANN ING DEPT, OH

\section{OKLAHOMA}

OKO01 - OKLAHOMA STATE DEPARTMENT OF HEALTH, OK OKO02 - OKLAHOMA WATER RESOURCES BOARD, OK OKOO3 - OKLAHOMA CLIMATOLOGICAL SURVEY, OK OK004 - OKLAHOMA DEPARTMENT OF TRANSPORTATION, OK 


\section{OREGON}

OROO1 - DEPARTMENT OF FOREST ENG, OREGON STATE UNIV, OR ORO03 - DOUGLAS COUNTY WATER RESOURCES SURVEY, OR OR0O4 - OREGON WATER RESOURCES DEPARTMENT, OR OR006 - PORTLAND GENERAL ELECTRIC COMPANY, OR OR007 - OREGON STATE FORESTRY DEPARTMENT, OR OR008 - THE CITY OF PENDLETON, OR OR009 - OREGON STATE DEPT OF FISH AND WILDLIFE, OR ORO10 - DELANO PHOTOGRAPHICS, INC, OR OROII - SPENCER B GROSS--AERIAL PHOTOGRAPHY, OR ORO12 - AERIAL MAPPING COMPANY OF OREGON, OR OROI3 - WAC CORPORATION, OR ORO14 - PORTLAND DEPARTMENT OF UTILITIES, OR OROI5 - OREGON STATE HIGHWAY DIVISION, OR OR016 - OREGON DEPT OF GEOLOGY \& MINERALS INDUSTRIES, OR OR017 - CHICKERING-GREEN EMPIRE, INC, OR ORO18 - CENTRAL BLUEPRINT COMPANY, AERO GRAPHICS DIV, OR OR019 - SKYVIEW AERIAL SURVEYS, INC, OR ORO20 - BEND MAPPING AND BLUEPRINTING, INC, OR

\section{PENNSYLVANI A}

PAOOI - PENNSYLVANIA DEPT OF ENVIRONMENTAL RESOURCES, PA 
PAO02 - KEYSTONE AERIAL SURVEYS, INC, PA

PAOO3 - METROPOLITAN EDISON COMPANY, PA

PAO04 - PENNSYLVANIA STATE DEPARTMENT OF MINES, PA

PAO05 - PHILADELPHIA ELECTRIC COMPANY, PA

PAO06 - PHILADELPHIA WATER DEPARTMENT, PA

PAO07 - ALLEGHENY COUNTY HEALTH DEPARTMENT, PA

PA008 - BRANDYWINE TRI-CONSERVANCY, PA

PAO09 - PA DEPT-TRANS, BUR OF DESIGN, PHOTO \& SURVEY, PA

PAOIO - ROY F WESTON, INC, PA

PAOII - CALGON CORPORATION, PA

PAOI2 - PENNSYLVANIA FISH COMMISSION, PA

PAOI3 - DELAWARE VALLEY REG PLANNING COMM, PA

PAOI4 - UNIVERSITY OF PITTSBURGH, PA

PAOI5 - MICHAEL BAKER, JR, INC, PA

PA016 - ALL STATES AERIAL SURVEY, PA

PAOI7 - QUINN\& ASSOCIATES, PA

PAOI8 - VERNON GRAPHICS, INC, PA

PA019 - R E WRIGHT ASSOCIATES, INC, PA

PA020 - GAI CONSULTANTS, PA

PA021 - CITY OF PITTSBURGH PLANNING DEPT, PA

PAO22 - ENVIRONMENTAL RESOURCES MANAGEMENT, INC, PA

PUERTO RICO

RQO0I - PUERTO RICO WATER RESOURCES AUTHORITY, PR 
RQ002 - WATER RESO RESE INST, UNIV OF PUERTO RICO, PR RQ003 - PUERTO RICO DEPARTMENT OF HEALTH, PR RQ004 - PUERTO RICO ENVIRONMENTAL QUALITY BOARD, PR RQ005 - PUERTO RICO DEPARTMENT OF AGRICULTURE, PR RQ006 - PUERTO RICO DEPT OF TRANSPORT \& PUBLIC WKS, PR RQ007 - PUERTO RICO AQUEDUCT \& SEWER AUTHORITY, PR RQ008 - PUERTO RICO DEPARTMENT OF NATURAL RESOURCES, PR

\author{
RHODE ISLAND \\ RIO0I - UNIVERSITY OF RHODE ISLAND, RI \\ RIO02 - RHODE ISLAND DEPT OF ENVIR MANAGEMENT, RI \\ RIO03 - RHODE ISLAND DEPT OF TRANSPORTATION, RI
}

SOUTH CAROLINA

SCOOI - AGRICULTURAL ENG DEPARTMENT, CLEMSON UNIV, SC SCO02 - GREENVILLE WATER SYSTEM, SC SCO03 - SPARTANBURG WATER WORKS, SC SCOO4 - SOUTH CAROLINA DEPT OF HEALTH \& ENVIR CONTROL,SC SCO05 - ENVIRONMENTAL SYSTEMS ENG DEPT, CLEMSON UNIV, SC SC006 - SOUTH CAROLINA STATE HIGHWAY DEPARTMENT, SC SC007 - SC LAND RESOURCES CONSERVATION COMMISSION, SC SC008 - SOUTH CAROLINA WATER RESOURCES COMMISSION, SC 
SCO09 - KERSHAW COUNTY TAX ASSESSOR, SC

SCO10 - ORANGEBURG COUNTY TAX ASSESSOR, SC

SCO11 - CALHOUN COUNTY TAX ASSESSOR, SC

SC012 - SUMTER COUNTY TAX ASSESSOR, SC

SC013 - SALUDA COUNTY TAX ASSESSOR, SC

SCO14 - LEXINGTON COUNTY TAX ASSESSOR, SC

SC015 - RICHLAND COUNTY TAX ASSESSOR, SC

SC016 - FAIRFIELD COUNTY TAX ASSESSOR, SC

SC017 - LEE COUNTY TAX ASSESSOR, SC

SCO18 - NEWBERRY COUNTY TAX ASSESSOR, SC

SCO19 - MARLBORO COUNTY TAX ASSESSOR, SC

SC020 - DILLON COUNTY TAX ASSESSOR, SC

SC021 - DARLINGTON COUNTY TAX ASSESSOR, SC

SCO22 - MARION COUNTY TAX ASSESSOR, SC

SC023 - HAMPTON COUNTY TAX ASSESSOR, SC

SC024 - EDGEFIELD COUNTY TAX ASSESSOR, SC

SC025 - CLARENDON COUNTY TAX ASSESSOR, SC

SC026 - CHESTERFIELD COUNTY TAX ASSESSOR, SC

SC027 - BARNWELL COUNTY TAX ASSEESSOR, SC

SC028 - SANTEE-COOPER PUBLIC SERVICE AUTHORITY, SC

SC029 - AIKEN COUNTY TAX ASSESSOR, SC

SC030 - BAMBERG COUNTY TAX ASSESSOR, SC

SC031 - GREENWOOD COUNTY TAX ASSESSOR, SC 
SC032 - CHESTER COUNTY TAX ASSESSOR, SC SC033 - LANCASTER COUNTY TAX ASSESSOR, SC SC034 - ALLENDALE COUNTY TAX ASSESSOR, SC SC035 - ABBEVILLE COUNTY TAX ASSESSOR, SC SC036 - PICKENS COUNTY TAX ASSESSOR, SC SC037 - ANDERSON COUNTY TAX ASSESSOR, SC SC038 - GREENVILLE COUNTY TAX ASSESSOR, SC SC039 - LAURENS COUNTY TAX ASSESSOR, SC SC040 - UNION COUNTY TAX ASSESSOR, SC SC04I - SPARTANBURG COUNTY TAX ASSESSOR, SC SC042 - CITY OF SPARTANBURG-ENGINEERING DEPT, SC SC043 - FLORENCE COUNTY TAX ASSESSOR, SC SC044 - SPARTANBURG COUNTY PLANNING COMMISSION, SC SC045 - SOUTH CAROLINA FORESTRY COMMISSION, SC SC046 - OCONEE COUNTY TAX ASSESSOR, SC SC047 - CITY OF ROCK HILL, SC SC048 - EDGEFIELD COUNTY EXTENSION SERVICE, SC SC049 - WILLIAMSBURG COUNTY TAX ASSESSOR, SC SC050 - CHEROKEE COUNTY TAX ASSESSOR, SC SC051 - CHARLESTON COUNTY TAX ASSESSOR, SC SC052 - DORCHESTER COUNTY TAX ASSESSOR, SC SC053 - COLLETON COUNTY TAX ASSESSOR, SC SC054 - BEAUFORT COUNTY TAX ASSESSOR, SC 
SC055 - SOUTH CAROLINA COASTAL COUNCIL, SC

SC056 - HORRY COUNTY TAX ASSESSOR, SC

SC058 - JASPER COUNTY TAX ASSESSOR, SC

SC059 - WACCAMAW REGIONAL PLANNING COUNCIL, SC

SC060 - CITY OF CHARLESTON PLANNING DEPT, SC

SC061 - GEORGIA PACIFIC TIMBER COMPANY, SC

SC062 - SC WILDLIFE \& MARINE RESOURCES DEPT, SC

SC063 - GREENVILLE COUNTY PLANNING COMMISSION, SC

SOUTH DAKOTA

SDOOI - WATER RESO RESEARCH INST, S DAK STATE UNIV, SD

SD002 - EAST DAKOTA CONSERVANCY SUBDISTRICT, SD

SD003 - SOUTH DAKOTA GEOLOGICAL SURVEY, SD

SDO04 - DAKOTA ENGINEERING COMPANY, SD

SD005 - SOUTH DAKOTA DEPT WATER \& NAT RESOURCES, SD

SD008 - SOUTH DAKOTA DEPT OF ENVIRONMENTAL PROT, SD

SDOO9 - SOUTH DAKOTA SCHOOL OF MINES AND TECHNOLOGY, SD

SDOIO - SOIL TESTING LAB, SOUTH DAKOTA STATE UNIV, SD

SDO11 - SOUTH DAKOTA STATE CHEMIST, UNIV OF S DAK, SD

SDOI2 - STATION BIOCHEMISTRY, S DAK STATE UNIV, SD

SDO13 - SOUTH DAKOTA DEPARTMENT OF TRANSPORTATION, SD 


\section{TENNESSEE}

TN001 - TENNESSEE WILDLIFE RESOURCES AGENCY, TN TNO02 - DIV-WATER QUAL CONT, TN DEPT OF PUB HEALTH, TN TN003 - CLEVELAND UTILITIES WATER DIVISION, TN TNO04 - CITY WATER COMPANY OF CHATTANOOGA, TN TN005 - BRISTOL WATER PLANT, TN TN006 - DEPARTMENT OF AGR ENG, UNIV OF TENN EXPER STA, TN TN007 - MEMPHIS LIGHT, GAS \& WATER DIVISION, TN TNO08 - WATER RESO REASEARCH CENTER, UNIV OF TENN, TN TN009 - TENN TECH UNIV-ENVIR BIOLOGY RESE CENTER, TN TNOIO - CONTINENTAL AERIAL SURVEYS, INC, TN TNOII - OAK RIDGE NATIONAL LABORATORY, TN TNOI 2 - TENNESSEE DEPARTMENT OF TRANSPORTATION, TN TNOI3 - TENNESSEE DEPARTMENT OF' CONSERVATION, TN

TEXAS

TX001 - TEXAS DEPARTMENT OF WATER RESOURCES, TX TX002 - TEXAS GENERAL LAND OFFICE, TX TX003 - TEXAS AIR CONTROL BOARD, TX TX004 - TEXAS FOREST SERVICE, TX TX005 - TEXAS INDUSTRIAL COMMISSION, TX TX006 - TEXAS DEPARTMENT OF HEALTH, TX 
TX008 - BUR OF ECONOMIC GEOLOGY (UNIV-TX AT AUSTIN), TX TX009 - RAILROAD COMMISSION OF TEXAS, TX

TX010 - TEXAS DEPARTMENT OF AGRICULTURE, TX

TX011 - TEXAS DEPT-HIGHWAYS \& PUBLIC TRANSPORTATION, TX

TX012 - TEXAS PARKS \& WILDLIFE DEPARTMENT, TX

TX013 - TEXAS STATE SOIL \& WATER CONSERVATION BOARD, TX

TXO15 - TEXAS NATURAL RESO INFORMATION SYSTEM, TX

TX016 - CITY OF AUSTIN, ENGINEERING DEPARTMENT, TX

TX017 - ESPEY, HUSTON \& ASSOCIATES, INC, TX

TX018 - TEXAS A \& M REMOTE SENSING CENTER, TX

TX019 - GRETEMAN AERIAL PHOTOMAPS, TX

TX020 - INTERNATIONAL AERIAL MAPPING COMPANY, TX

TX021 - UNITED AERIAL MAPPING, TX

TX022 - TEXAS COASTAL AND MARINE COUNCIL, TX

TX023 - AERO SERVICE CORPORATION, TX

TX024 - HOUSTON LIGHTING AND POWER COMPANY, TX

TX025 - TEXAS A\&M UNIVERSITY, CHEMISTRY DEPT, TX

TX026 - PETROLEUM INFORMATION CORPORATION, TX

TX027 - WILLIAMS-STACKHOUSE INC AERIAL MAP MOSAICS, TX

TX028 - RADIAN CORPORATION, TX

TX029 - AERO COLOR, TX

TX030 - ADAMS AERIAL SURVEYS, TX

TX031 - TOBIN RESEARCH, TX 
TX032 - METROPOLITAN AERIAL SURVEYS, TX

TX033 - NORTHERN TECHNICAL SERVICES, TX

TX034 - SOUTHERN METHODIST UNIV SCIENCE LIBRARY, TX

TX035 - JERRY FOSTER \& COMPANY, TX

TX036 - LANDIS AERIAL PHOTO, TX

TX037 - MAPCO, TX

TX038 - MPSI MAPS, INC, TX

TX039 - K. M. NG \& ASSOCIATES, INC

TX040 - THOMAS G. GEBHARD, CONSULTING ENGINEER, TX

TX04I - HOUSTON PUBLIC LIBRARY, TX

TX042 - WILSON AERIAL SURVEYS, TX

UTAH

UTOOI - UTAH STATE HEALTH DEPARTMENT, UT

UT002 - METROPOLITAN WATER DIST OF SALT LAKE CITY, UT

UT003 - DIV-FISH \& GAME, UTAH DEPT OF NAT RESOURCES, UT

UTO04 - SALT LAKE COUNTY WATER CONSERVANCY DISTRICT, UT

UT005 - SALT LAKE CITY WATER SUPPLY \& WATERWORKS, UT

UT006 - OGDEN BAY WATERFOWL MANAGEMENT AREA, UT

UT007 - CLEAR LAKE WATERFOWL MANAGEMENT AREA, UT

UT008 - UTAH GEOLOGICAL \& MINERALOGICAL SURVEY, UT

UTO09 - OGDEN RIVER WATER USERS, UT

UTO1O - WEBER DISTRIBUTION SYSTEM, UT 
UTO1I - UTAH WATER RESEARCH LAB, UTAH STATE UNIV, UT UTO12 - SALT LAKE COUNTY RECREATION DEPARTMENT, UT UT013 - DIV OF WATER RIGHTS, UTAH DEPT OF NAT RESO, UT UTO14 - MOUNTAINLAND ASSOCIATION OF GOVERNMENTS, UT UTO15 - TEMPLETON, LINK \& ALSUP ASSOCIATION, UT UTOI6 - CENTER FOR WATER RESO RESE, UTAH STATE UNIV, UT UTOI7 - UINTEX CORPORATION, UT UTO18 - UTAH DEPARTMENT OF TRANSPORTATION, UT

\section{VERMONT}

VTOOI - STATE OF VERMONT, AGCY OF ENVIR CONSERVATION, VT VTOO2 - VERMONT DEPARTMENT OF HEALTH, VT VTOO3 - VERMONT WATER RESO RESE CTR, UNIV OF VERMONT, VT VTO04 - VERMONT DEPARTMENT OF WATER RESOURCES, VT VTOO5 - VERMONT DEPARTMENT OF FISH AND GAME, VT VTOO6 - UNIVERSITY OF VERMONT, VT VTO07 - VERMONT AGENCY OF TRANSPORTATION, VT

\section{VIRGIN ISLANDS (US)}

VQ00I - VIRGIN ISLANDS DEPARTMENT OF PUBLIC WORKS, VI

\section{VIRGINIA}

VAO01 - VIRGINIA STATE WATER CONTROL BOARD, VA 







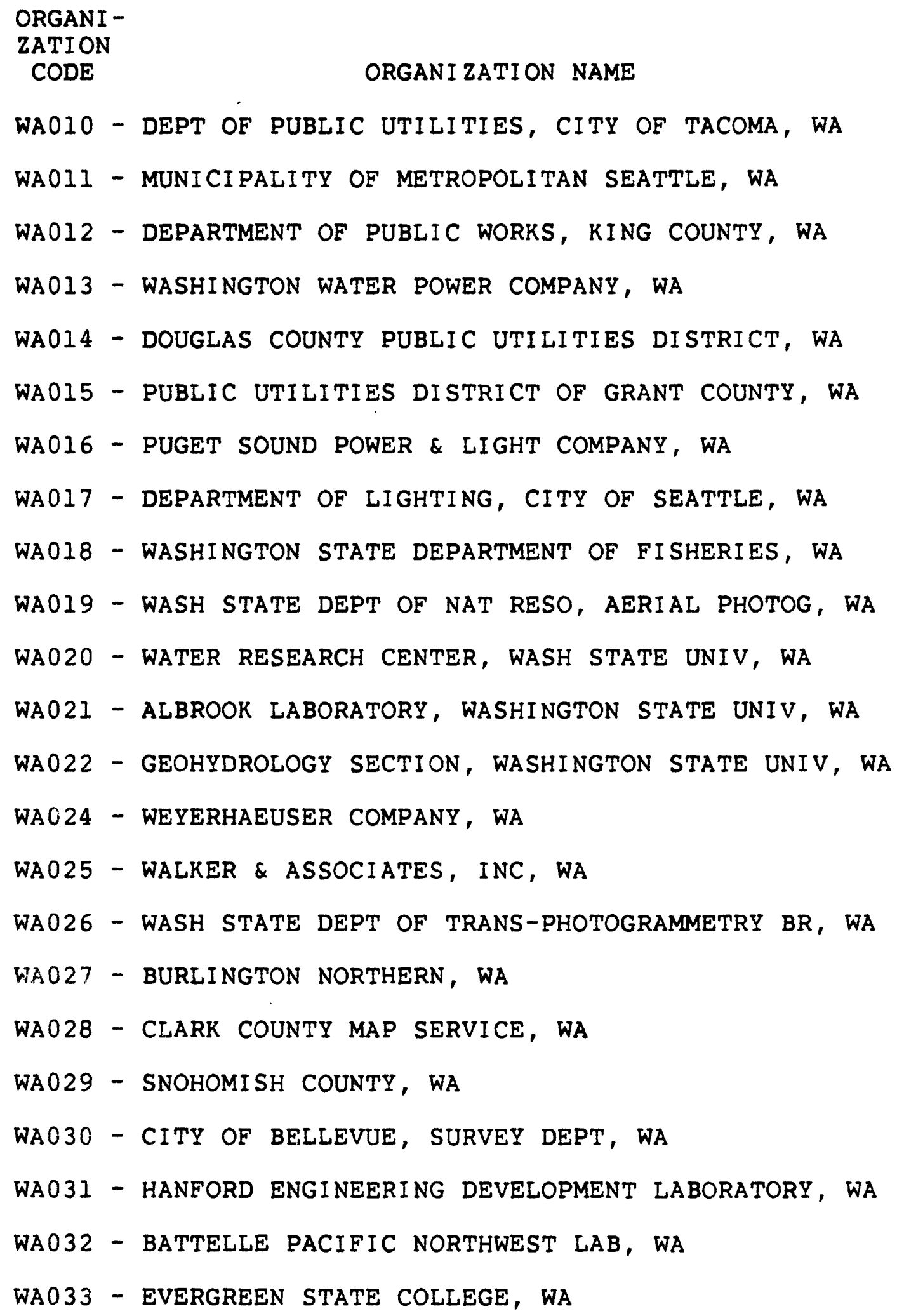


WA034 - WASH STATE UNIV, DEPT OF CIVIL ENG, WA WA035 - S.J. GROVES \& SONS COMPANY, WA

\section{WEST VIRGINIA}

WVOOI - DIV OF WATER RESO, W VA DEPT OF NAT RESO, WV WV002 - SANITARY ENG DIV, W VA DEPARTMENT OF HEALTH, WV WV003 - W VA GEOLOGICAL \& ECONOMIC SURVEY, WV WV004 - WEST VIRGINIA DEPARTMENT OF HIGHWAYS, WV WV005 - WEST VIRGINIA ENVIRONMENTAL HEALTH SERVICE, WV

\section{WI SCONSIN}

WIOO1 - WISCONSIN DEPARTMENT OF NATURAL RESOURCES, WI WIO02 - EAST CENTRAL WISCONSIN REGIONAL PLANNING COM, WI WI003 - DAIRYLAND POWER COOPERATIVE, WI WIOO4 - NORTHERN STATES POWER COMPANY, WI WIOO5 - WISCONSIN STATE LABORATORY OF HYGIENE, WI WI006 - WISCONSIN PUBLIC SERVICE COMMISSION, WI WI007 - UNIV OF WIS, COLLEGE OF ENG, K F WENDT LAB, WI WI008 - SOUTHEASTERN WISCONSIN REG PLANNING COMM, WI WI009 - WISCONSIN GEOLOGICAL SURVEY, WI WI0I0 - DANE COUNTY REG PLANNING COMMISSION, WI WIOII - BAY-LAKE REGIONAL PLANNING COMMISSION, WI 
WI0I2 - UNIVERSITY OF WISCONSIN SEA GRANT PROGRAM, WI WIOI4 - WISCONSIN DEPARTMENT OF TRANSPORTATION, WI WI015 - STATE CARTOGRAPHER'S OFFICE, UNIV OF WISC, WI WIOI6 - REMOTE SENSING DATA CENTER, UNIV OF WIS, WI WI017 - ENVIRO ENERGY TECHNOLOGY CENTER (REXNORD), WI

WYOMING

WYOOI - CITY OF CASPER BOARD OF PUBLIC UTILITIES, WY WY002 - SHERIDAN WATER DEPARTMENT, WY WY003 - WYOMING STATE ENGINEER, WY WYOO4 - WATER RESOURCES RESE INST, UNIV OF WYOMING, WY WY005 - WYOMING HIGHWAY DEPARTMENT, WY WY006 - WYOMING DEPARTMENT OF ENVI RONMENTAL QUALITY, WY 
IDENTIFICATION CODES FOR ORGANIZATIONS IN

ALPHABETICAL ORDER BY ORGANIZATION NAME

ORGANI -

ZATION

CODE

ORGANIZATION NAME

FOREIGN ORGANIZATIONS

MXXO1 - AQUA MEX, SA, MEXICO

BRXO1 - COMPANHIA AUXIL-EMPRESAS ELETRICAS BRASILEIRAS

CAX03 - DEPT OF MINES, RESO \& ENVIRON MANAGEMENT, CANADA

CAX02 - INLAND WATERS DIRECTORATE, WATER QUAL BR, CANADA

CAXO1 - INLAND WATERS DIRECTORATE, WATER RESO BR, CANADA

CAX04 - INLAND WTR DIRECTORATE-DEPT OF ENVIR, CANADA

CHXOI - INTERNAT INFORMATION SERVICE LTD, (HONG KONG)CH

FRXOI - INTERNAT COUNCIL OF SCIENTIFIC UNIONS, (PARIS) FR

CAX05 - MINISTRY OF THE ENVIRONMENT--CANADA

INXOI - NATIONAL INSTITUTE OF HYDROLOGY (ROORKEE, INDIA)

CAXO6 - SMALL HYDRO-ELECTRICS CANADA, LTD

SFXO1 - SOUTH AFRICAN WAT INFORMATION CENTRE (PRETORIA)

FEDERAL ORGANIZATIONS

USARS - AGRICULTURAL RESEARCH SERVICE

USAF - AIR FORCE

USAPA - ALASKA POWER ADMINISTRATION

USAHS - ARMY HEALTH SERVICE 


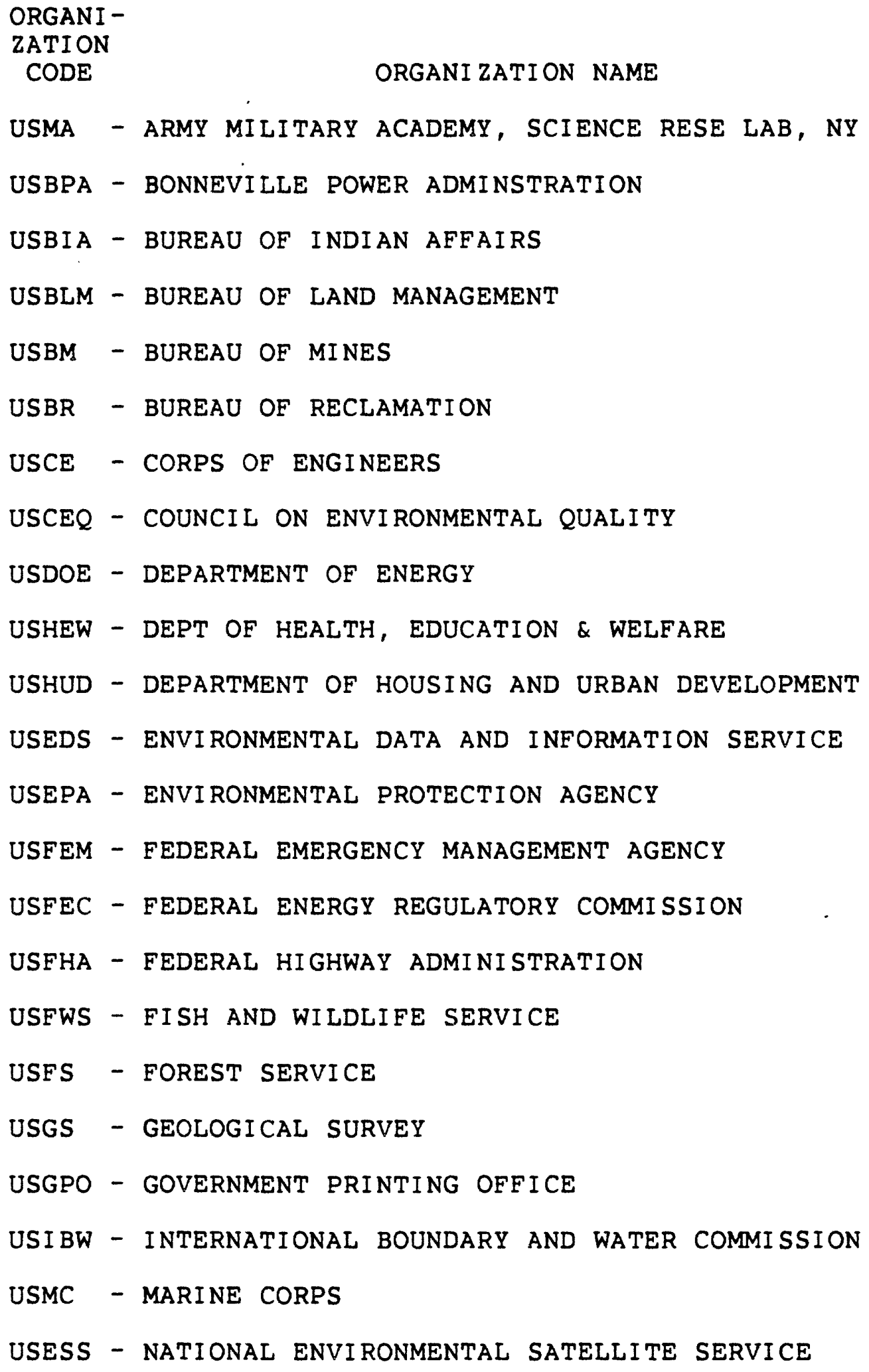




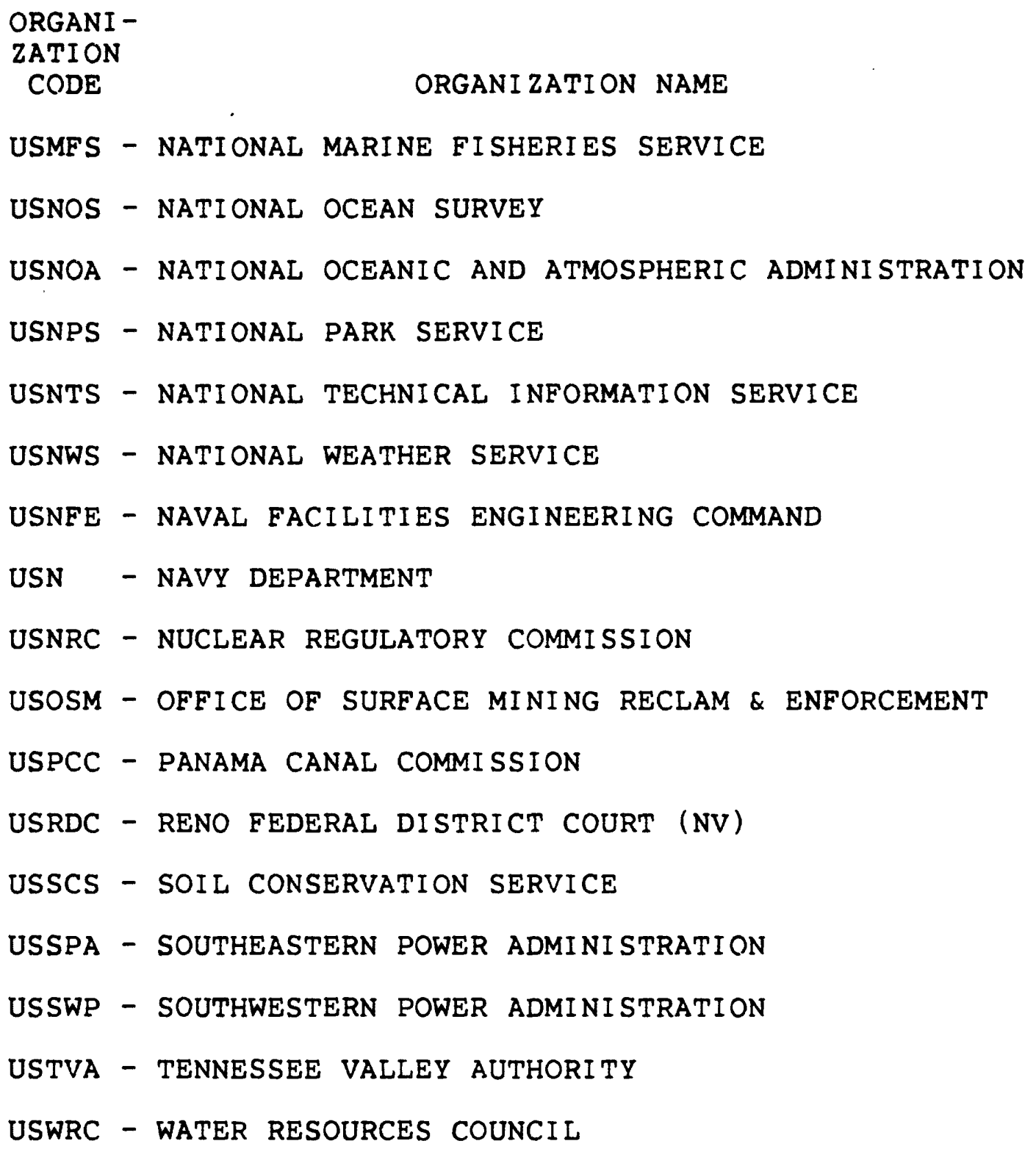

ORGANIZATIONS WITH NATIONAL OR MULTISTATE JURISDICTION BCC - BOISE CASCADE TIMBER COMPANY

$\mathrm{CHMH}-\mathrm{CH} 2 \mathrm{M} / \mathrm{HILL}$

DMI - DAMES \& MOORE, INC

DRBC - DELAWARE RIVER BASIN COMMISSION 


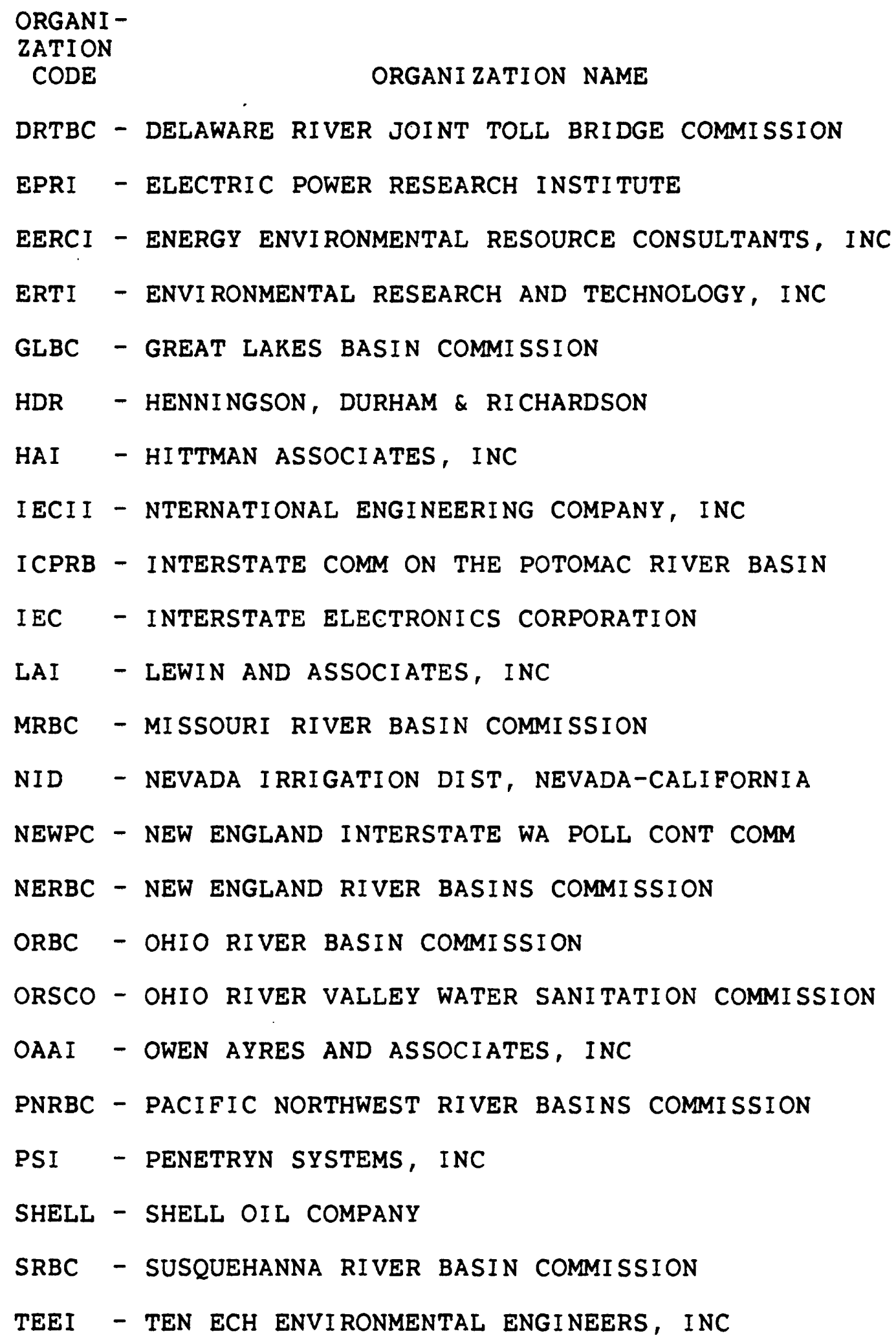


ORGANI -

ZATION

CODE

ORGANI ZATION NAME

UCRBC - UPPER COLORADO RIVER COMMISSION

UMRBC - UPPER MISSISSIPPI RIVER BASIN COMMISSION

WETI - WATER ENGINEERING AND TECHNOLOGY, INC

WMPC - WISCONSIN ELECTRIC POWER COMPANY

NON-FEDERAL ORGANIZATIONS WITH STATE OR LOCAL JURISDICTION

\section{ALABAMA}

AL007 - ALABAMA DEPARTMENT OF PUBLIC HEALTH

AL006 - ALABAMA STATE HIGHWAY DEPARTMENT, AL

ALO02 - ALABAMA WATER IMPROVEMENT COMMISSION, AL

AL004 - ATLANTIC AERIAL SURVEYS, INC, AL

AL003 - AUBURN UNIV WATER RESOURCES RESEARCH INST, AL

ALO01 - GEOLOGICAL SURVEY OF ALABAMA, AL

ALO05 - WEST ALABAMA PLANNING \& LAND DEVEL COUNCIL, AL

\section{ALASKA}

AK007 - AIR PHOTO TECH, INC, AK

AKO02 - ALASKA DEPT OF ENVIRONMENTAL CONSERVATION, AK

AK008 - ALASKA DEPT OF FISH AND GAME, AK

AKOOI - ALASKA DEPT OF TRANS \& PUBLIC FACILITIES, AK

AKO04 - ALASKA DEPT OF NATURAL RESOURCES, AK

AK003 - CHUGACH ELECTRIC ASSOCIATION, AK 
AKO05 - NORTH PACIFIC AERIAL SURVEYS, INC, AK AK006 - STATE OF ALASKA, PIPELINE COORDINATORS OFFICE,AK AK009 - UNIVERSITY OF ALASKA, AK

\section{AMERICAN SAMOA}

AQOOI - DEPT OF PUBLIC WORKS, GOVT OF AMERICAN SAMOA

ARI ZONA

AZOI9 - AIROGRAPHICS CORPORATION, AZ AZ007 - ARIZONA DEPARTMENT OF HEALTH, AZ AZOI7 - ARIZONA DEPARTMENT OF TRANSPORTATION, AZ AZO14 - ARIZONA DEPT OF WATER RESOURCES, AZ AZ003 - ARI ZONA GAME AND FISH DEPARTMENT, AZ AZ009 - ARIZONA RESOURCES INFORMATION SYSTEM, AZ AZ021 - ARIZONA STATE UNIVERSITY, AZ AZ013 - CITY OF TUCSON WATER AND SEWER DEPARTMENT, AZ AZ010 - COOPER AERIAL SURVEY COMPANY, AZ AZ016 - FAIRCHILD NATIONAL, INC, AZ AZ005 - GILA WATER COMMISSIONER, AZ AZ020 - KENNY AERIAL MAPPING, INC, AZ AZ018 - LANDIS AERIAL SURVEYS, AZ AZ004 - MARICOPA COUNTY MUN WATER CONS DISTRICT \#I, AZ 
AZOI5 - MOTOROLA AERIAL REMOTE SENSING, INC, AZ

AZ002 - ROOSEVELT IRRIGATION DISTRICT, AZ

AZOII - SALT RIVER PROJECT, AZ

AZ006 - SALT RIVER VALLEY WATER USERS ASSOC, AZ

AZ012 - TOUPS CORPORATION, AZ

AZ008 - UNIV OF ARI Z, COLLEGE-BUSINESS \& PUBLIC ADMIN,AZ

AZOOI - ARI ZONA UNIV, WATER RESOURCES RESE CENTER, AZ

ARKANSAS

AROOI - ARKANSAS DEPT OF HEALTH, BUR OF ENVIR ENG, AR

AROO3 - ARKANSAS DEPT-POLLUTION CONTROL AND ECOLOGY, AR

AROO2 - ARKANSAS GAME AND FISH COMMISSION, AR

AR004 - ARKANSAS GEOLOGICAL COMMISSION, AR

AR005 - ARKANSAS HIGHWAY DEPARTMENT, AR

AR007 - ARKANSAS POWER AND LIGHT, AR

AR008 - ARKANSAS SOIL \& WATER CONSERVATION COMM, AR AR006 - UNIVERSITY OF ARKANSAS, AR

\section{CALI FORNI A}

CA173 - AERIAL FOTOBANK, CA

CAOI4 - AERIAL MAP INDUSTRIES, CA

CAI94 - AERO-GEODETIC CORPORATION, CA 
CA190 - AERO PHOTOGRAPHERS, INC, CA

CA198 - AERO TECH SURVEYS, CA

CA199 - AIR DATA SYSTEMS, CA

CA142 - AIR PHOTO COMPANY, INC, CA

CA047 - ALAMEDA CNTY FLOOD CONTROL \& WATER CONS DIST, CA

CA097 - ALAMEDA COUNTY HEALTH DEPARTMENT, CA

CAI09 - ALAMEDA COUNTY OFFICE OF EDUCATION, CA

CA006 - ALAMEDA COUNTY WATER DISTRICT, CA

CA157 - ALPAUGH IRRIGATION DISTRICT, CA

CA128 - AMERICAN AERIAL SURVEYS INC-NORTHERN CALIF, CA

CAI36 - AMERICAN AERIAL SURVEYS INC-SOUTHERN CALIF, CA

CA179 - ARVIN-EDISON WATER STORAGE DISTRICT, CA

CAI95 - ASIA MAPPING, INC, CA

CA236 - BIG BASIN WATER COMPANY, CA

CA219 - BIG BEAR MUNICIPAL WATER DISTRICT, CA

CA084 - BODEGA BAY MARINE LAB, UNIV OF CA (BERKELEY), CA

CA202 - BOYLE ENGINEERING CORPORATION, CA

CA165 - BROWN AND CALDWELL, CA

CA036 - BUENA VISTA WATER STORAGE DISTRICT, CA

CA072 - CABRILLO MARINE MUSEUM, CA

CA223 - CABRILLO SAND AND GRAVEL, CA

CA193 - CAL AERO PHOTO, CA

CA249 - CALAVERAS PUBLIC UTILITY DISTRICT, CA 
CA004 - CALIF DEPT TRANS, DIV-HIGHWAYS, PHOTOGRAM BR,CA

CA063 - CALIF UNIV-DAVIS-DIV OF ENVIR STUDIES, CA

CA235 - CALIFORNIA-AMERICAN WATER COMPANY, CA

CA003 - CALIFORNIA DEPARTMENT OF FISH \& GAME, CA

CAIII - CALIFORNIA DEPARTMENT OF HEALTH, CA

CAO01 - CALIFORNIA DEPARTMENT OF WATER RESOURCES, CA

CA225 - CALIFORNIA DEPT OF PARKS AND RECREATION, CA

CA073 - CALIFORNIA INSTITUTE OF TECHNOLOGY, CA

CA076 - CALIFORNIA STATE COLLEGE AT FULLERTON, CA

CA075 - CALIFORNIA STATE UNIVERSITY AT LONG BEACH, CA

CA185 - CALIFORNIA STATE UNIVERSITY AT NORTHRIDGE, CA

CAO09 - CALIFORNIA STATE WATER RESOURCES CONTROL BRD, CA

CA164 - CALIFORNIA WATER LABS, CA

CA241 - CALIFORNIA WATER SERVICE COMPANY, CA

CA229 - CALISTOGA CITY WATER DEPT, CA

CAIIO - CARTWRIGHT AERIAL SURVEYS, INC, CA

CA024 - CENTRAL CALIFORNIA IRRIGATION DISTRICT, CA

CAII4 - CENTRAL COAST REG, CA REG WTR QUAL CONT BRD, CA

CAI16 - CENTRAL VALLEY REG, CA REG WTR QUAL CONT BRD, CA

CA035 - CITY OF BAKERSFIELD DEPT OF WATER, CA

CA251 - CITY OF DOS PALOS, CA

CA216 - CITY OF ESCONDIDO WATER \& SEWER MAINTENANCE, CA

CAI47 - CITY OF LONG BEACH ENGINEER, CA 
CA086 - CITY OF LONG BEACH HEALTH DEPARTMENT, CA

CA213 - CITY OF OCEANSIDE WATER \& SEWER DEPT, CA

CA208 - CITY OF SAN DIEGO WATER UTILITIES DEPT, CA

CA237 - CITY OF SANTA CRUZ, CA

CA227 - CITY OF ST HELENA, CA

CA015 - COACHELLA VALLEY COUNTY WATER DISTRICT, CA

CA244 - COASTSIDE COUNTY WATER DEPARTMENT, CA

CAII7 - COL R BASIN REGION, CA REG WTR QUAL CONT BRD, CA

CAI3I - CONTRA COSTA WATER DISTRICT, CA

CAOIO - COUNTY OF LOS ANGELES, SURVEY DIVISION, CA

CAI35 - COUNTY OF SAN DIEGO, DEPT OF TRANSPORTATION, CA

CA247 - CUESTA LA HONDA GUILD, INC, CA

CAO34 - DELANO-EARLIMART IRRIGATION DISTRICT, CA

CA052 - DESERT WATER AGENCY, CA

CA080 - DIABLO VALLEY COLLEGE, CA

CA025 - EAST BAY MUNICIPAL UTILITY DISTRICT, CA

$\because \measuredangle 7$ - EL NIDO IRRIGATION DISTRICT, CA

CAI91 - ERTEC AIRBORNE SYSTEMS, CA

CA053 - ESCONDIDO MUTUAL WATER COMPANY, CA

CAI78 - $M C$ CORPORATION, CA

CA253 - FERN VALLEY STATE WATER DISTRICT, CA

CA245 - FOREST LAKES MUTUAL WATER COMPANY, CA

CA174 - FOSTER FARMS (DELHI), CA 
ORGANI -

ZATION

CODE

ORGANIZATION NAME

CAI6I - FRESNO CITY DEPT OF PUBLIC WORKS, CA

CA160 - FRESNO COUNTY DEPT OF HEALTH, CA

CA03I - FRESNO IRRIGATION DISTRICT, CA

CA126 - GENGE AERIAL SURVEYS, INC, CA

CA256 - GIBBS\& HILL, INC, CA

CA182 - GRENSTED PHOTOGRAPHY, CA

CA255 - HARL PUGH \& ASSOCIATES, CA

CA0?9 - HETCH HETCHY WATER SUPPLY, SAN FRANCISCO, CA

CA233 - HIRSCH AND COMPANY, CA

CA196 - HOGAN-SCHOCH \& ASSOCIATES, INC, CA

CAI02 - HOPKINS MARINE STATION LIBRARY, CA

CAI48 - HORIZON SURVEYS, CA

CA098 - HUMBOLDT-DEL NORTE CNTY PUBLIC HEALTH DEPT, CA

CA108 - HUMBOLDT STATE UNIV, DEPT OF OCEANOGRAPHY, CA

CA107 - HUMBOLDT STATE UNIVERSITY, DEPT OF BIOLOGY, CA

CA106 - HUMBOLDT STATE UNIVERSITY, DEPT OF FISHERIES, CA

CAI4I - HYDRO RESEARCH SCIENCE, CA

CAI44 - I K CURTIS SERVICES, INC, CA

CA133 - IMPERIAL COUNTY HEALTH DEPARTMENT, CA

CA139 - KAISER ENGINEERS, CA

CA032 - KAWEAH \& ST JOHNS WATER ASSOCIATION, CA

CA140 - KENNEDY ENGINEERING, CA

CA203 - KERN COUNTY DEPT OF PUBLIC WORKS, CA 
ORGANI-

ZATION

CODE

ORGANI ZATION NAME

CA166 - KERN COUNTY WATER AGENCY, CA

CA159 - KINGS COUNTY WATER DISTRICT, CA

CA061 - KINGS RIVER CONSERVATION DISTRICT, CA

CA030 - KINGS RIVER WATER ASSOCIATION, CA

CA181 - KNUTSON AERIAL PHOTOGRAPHY, CA

CA062 - LA CANADA IRRIGATION DISTRICT, CA

CA064 - LAHONTAN, CA REG WATER QUAL CONT BRD, CA

CA234 - LAKESIDE PARK ASSOCIATION, CA

CA153 - LAWRENCE BERKELEY LABORATORY, CA

CA240 - LOMPICO COUNTY WATER DISTRICT, CA

CA214 - LOS ALISOS WATER DISTRICT, CA

CA051 - LOS ANGELES CITY DEPT OF WATER \& POWER, CA

CAOO5 - LOS ANGELES COUNTY FLOOD CONTROL DISTRICT, CA

CA089 - LOS ANGELES COUNTY HEALTH DEPARTMENT, CA

CAI15 - LOS ANGELES REg, CA REG WTR QUAL CONT BRD, CA

CAI46 - LYON ASSOCIATES, INC, AERIAL MAPPING DIV, CA

CAO28 - MADERA IRRIGATION DISTRICT, CA

CA083 - MARIN COLLEGE, CA

CA044 - MARIN MUNICIPAL WATER DISTRICT, CA

CA250 - MARIPOSA PUBLIC UTILITY DISTRICT, CA

CA137 - MC LAREN ENVIRONMENTAL ENGINEERING, CA

CA172 - MENDOCINO COUNTY PUBLIC HEALTH DEPARTMENT, CA

CA162 - MERCED COUNTY DEPT OF HEALTH, CA 
ORGANI -

ZATION

CODE
ORGANIZATION NAME

CAOI9 - MERCED IRRIGATION DISTRICT, CA

CA082 - MERRITT COLLEGE, CA

CAI43 - METREX MANAGEMENT CORPORATION, CA

CA043 - METROPOLITAN WATER DIST OF SOUTHERN CALIF, CA

CA026 - MODESTO IRRIGATION DISTRICT, CA

CA050 - MONTAGUE WATER CONSERVATION DISTRICT, CA

CA041 - MONTECITO COUNTY WATER DISTRICT, CA

CAO39 - MONTEREY CNTY FLOOD CONT \& WATER CONS DIST, CA

CA224 - MONTEREY COUNTY DEPT OF PUBLIC WORKS, CA

CA093 - MONTEREY COUNTY HEALTH DEPARTMENT, CA

CAO23 - MOSQUITO IRRIGATION DISTRICT, CA

CAI03 - MOSS LANDING MARINE LABORATORY, CA

CA228 - MT HERMON ASSOCIATION, CA

CAII2 - N COAST REGION, CA REGIONAL WTR QUAL CONT BRD,CA

CA087 - NAPA CNTY FLOOD CONT \& WA CONS DIST, CA

CA201 - NATIONAL AERO MAPPERS, CA

CA232 - CALIF UNIV-IRVINE, WATER RESO LAB, CA

CA242 - NORTH COAST COUNTY WATER DISTRICT, CA

CA155 - NORTH KERN WATER STORAGE DISTRICT, CA

CA045 - NORTH MARIN COUNTY WATER DISTRICT, CA

CA077 - OCCIDENTAL COLLEGE, CA

CA218 - ORANGE COAST COLLEGE, CA

CA069 - ORANGE COUNTY DEPARTMENT OF EDUCATION, CA 


\section{ORGANI -}

ZATION

CODE

ORGANI ZATION NAME

CA018 - ORANGE COUNTY ENVIR MANAGEMENT AGENCY, CA

CA059 - ORANGE COUNTY HEALTH DEPARTMENT, CA

CA022 - OROVILLE-WYANDOTTE IRRIGATION DISTRICT, CA

CA221 - OTAY MUNICIPAL WATER DISTRICT, CA

CA012 - PACIFIC AERIAL SURVEYS, CA

CA138 - PACIFIC ENVIRONMENTAL LABORATORY, CA

CA176 - PACIFIC FIBREBOARD, CA

CA065 - PACIFIC GAS \& ELECTRIC COMPANY, CA

CA129 - PACIFIC MARINE STATION, CA

CA067 - PACIFIC POWER \& LIGHT, CA

CA149 - PACIFIC WESTERN AERIAL SURVEYS, CA

CA200 - PICTORIAL CRAFTS, INC, CA

CAI25 - PLACER COUNTY, DEPT OF PUBLIC WORKS, CA

CA254 - PLACER COUNTY WATER AGENCY, CA

CA209 - POWAY MUNICIPAL WATER DISTRICT, CA

CA215 - RAINBOW MUNICIPAL WATER DISTRICT, CA

CA243 - REDWOOD MUTUAL WATER COMPANY, CA

CA197 - RICHARD B DAVIS COMPANY, CA

CA057 - RIVERSIDE CNTY FLOOD CONT \& WATER CONS DIST, CA

CA132 - RIVERSIDE COUNTY DEPARTMENT OF PUBLIC HEALTH, CA

CAIOO - S CALIFORNIA COASTAL WATER RESEARCH PROJECT, CA

CA134 - SACRAMENTO CNTY DEPT-PUB WKS, WATER RESO DIV, CA

CA188 - SACRAMENTO MUNICIPAL UTILITY DISTRICT, CA 
ORGANI -

ZATION

CODE

ORGANI ZATION NAME

CA055 - SAN ANTONIO WATER COMPANY, CA

CAI7I - SAN BENITO CNTY WATER CONS \& FLOOD CONT DIST, CA

CA054 - SAN BERNARDINO COUNTY FLOOD CONTROL DISTRICT, CA

CA088 - SAN DIEGO COUNTY HEALTH DEPARTMENT, CA

CAOI7 - SAN DIEGO DEPT OF SANIT \& FLOOD CONTROL, CA

CAII9 - SAN DIEGO REGION, CA REGIONAL WTR CONT BRD, CA

CA070 - SAN DIEGO STATE UNIVERSITY, CA

CA096 - SAN FRANCISCO CITY/CNTY BUR-ENVIR HEALTH SERV,CA

CA085 - SAN FRANCISCO PORT AUTHORITY, CA

CA226 - SAN FRANCISCO WATER DISTRICT, CA

CA068 - SAN GABRIEL ELECTRIC COMPANY, CA

CA163 - SAN JOAQUIN COUNTY DEPT OF PUBLIC WORKS, CA

CA246 - SAN JOSE WATER WORKS, CA

CA230 - SAN JUAN SUBURBAN WATER DISTRICT, CA

CA002 - SAN-LO AERIAL SURVEYS INC, CA

CA239 - SAN LORENZO VALLEY COUNTY WATER DISTRICT, CA

CA040 - SAN LUIS OBISPO CNTY FLOOD CONT-WA CONS DIST, CA

CA092 - SAN LUIS OBISPO COUNTY HEALTH AGENCY, CA

CA168 - SAN MATEO COUNTY DEPT OF PUBLIC WORKS, CA

CA095 - SAN MATEO COUNTY HEALTH DEPARTMENT, CA

CAI18 - SANTA ANA REGION, CA REGIONAL WTR CONT BRD, CA

CA042 - SANTA BARBARA CNTY FLOOD CONT \& WA CONS DIST, CA

CA091 - SANTA BARBARA COUNTY HEALTH DEPARTMENT, CA 
ORGANI -

ZATION

CODE

ORGANI ZATION NAME

CA074 - SANTA CATALINA MARINE LABORATORY, CA

CA151 - SANTA CLARA CITY WATER DEPARTMENT, CA

CA048 - SANTA CLARA VALLEY WATER DISTRICT, CA

CA094 - SANTA CRUZ COUNTY HEALTH DEPARTMENT, CA

CA222 - SANTA FE IRRIGATION DISTRICT, CA

CA217 - SANTA MARGARITA WATER DISTRICT, CA

CA038 - SAUSALITO IRRIGATION DISTRICT, CA

CA121 - SCRIPPS INST OF OCEANOGRAPHY, SHORE TEMP DATA,CA

CA122 - SCRIPPS INSTITUTE OF OCEANOGRAPHY, CA

CA177 - SELMA-KINGSBURG-FOWLER SANITATION DISTRICT, CA

CA113 - SF BAY REGION, CA REGIONAL WTR QUAL CONT BRD, CA

CA071 - SHELTER ISLAND LOCKHEED OCEANOGRAPHY LAB, CA

CA013 - SIERRA ENVIRONMENTAL MONITORING, CA

CA046 - SONOMA CNTY FLOOD CONTROL \& WATER CONS DIST, CA

CA170 - SONOMA COUNTY DEPARTMENT OF PLANNING, CA

CAO99 - SONOMA COUNTY PUBLIC HEALTH SERVICE, CA

CA186 - SOQUEL CREEK COUNTY WATER DISTRICT, CA

CA211 - SOUTH COAST COMPANY WATER DISTRICT, CA

CA066 - SOUTHERN CALIFORNIA EDISON COMPANY, CA

CA175 - STANISLAUS COUNTY DEPT OF ENVIRON RESOURCES, CA

CA212 - SWEETWATER AUTHORITY, CA

CA127 - TELEDYNE GEOTRONICS, CA

CA056 - TEMESCAL WATER COMPANY, CA 
ORGANI -

ZATION

CODE

ORGANI ZATION NAME

CA037 - TERRA BELLA IRRIGATION DISTRICT, CA

CA150 - TETRA TECH INC, CA

CA184 - TOWILL, INC, CA

CA021 - TRI-DAM PROJ, OAK \& SAN JOAQUIN IRR DIST, CA

CAI56 - TULARE COUNTY DEPT OF PUBLIC WORKS, CA

CA033 - TULARE IRRIGATION DISTRICT, CA

CAI58 - TULARE LAKE DRAINAGE DISTRICT, CA

CA049 - TULE IRRIGATION DISTRICT, CA

CA020 - TURLOCK IRRIGATION DISTRICT, CA

CA130 - UNIV CA AGR EXT, FARM \& HOME ADVISORS OFEICE, CA

CA248 - UNION PUBLIC UTILITY DISTRICT, CA

CA060 - UNITED WATER CONSERVATION DISTRICT, CA

CA08I - UNIV CALIFORNIA-BERKELEY, RICHMOND FIELD STA, CA

CA124 - UNIV CALIF-SANTA BARBARA, DEPT OF BIOLOGY, CA

CAIOI - UNIV OF CALIF AT LOS ANGELES, DEPT OF BIOLOGY,CA

CAI05 - UNIV OF CALIF-BERKELEY-LAWRENCE IIVERMORE LAB,CA

CAI04 - UNIV OF CALIF-BERKELEY-SANIT RESE LAB, CA

CA078 - UNIVERSITY OF CALIFORNIA AT SANTA CRUZ, CA

CA123 - UNIVERSITY OF CALIFORNIA-SANTA BARBARA, CA

CA079 - UNIVERSITY OF SANTA CLARA, CA

CA120 - UNIVERSITY OF SOUTHERN CALIFORNIA, CA

CA192 - UPDATA PUBLICATIONS, INC, CA

CA167 - VARA SYSTEMS, CA 
ORGANI -

ZATION

CODE

ORGANI ZATION NAME

CAOI6 - VENTURA COUNTY FLOOD CONTROL DISTRICT, CA

CA090 - VENTURA COUNTY HEALTH DEPARTMENT, CA

CA058 - VENTURA RIVER MUNICIPAL WATER DISTRICT, CA

CA252 - VISTA IRRIGATION DISTRICT, CA

CA145 - VTN CONSOLIDATED, INC, CA

CAOO7 - WATER RESO DIV, SACRAMENTO DEPT OF PUB WKS, CA

CA238 - WATSONVILLE, CITY OF, CA

CA169 - WESTERN AERIAL PHOTOS, INC, CA

CA152 - WESTERN AERIAL SURVEY, CA

CA154 - WHEELER RIDGE-MARICOPA WATER STORAGE DIST, CA

CA008 - WHITEWATER MUTUAL WATER COMPANY, CA

CA183 - WILSON'S PHOTOGRAPHY, CA

CA180 - WILVERT-PENCEK PHOTOGRAPHIC SERVICES, CA

CA220 - YUCAIPA VALLEY COUNTY WATER DISTRICT, CA

\section{COLORADO}

COO13 - AGRIC EXPER STA, COLORADO STATE UNIV, CO COOO4 - BOULDER CITY-COUNTY HEALTH DEPARTMENT, CO CO020 - COLORADO DEPARTMENT OF HIGHWAYS, CO CO006 - COLORADO DEPARTMENT OF NATURAL RESOURCES, CO COOO2 - COLO DIV OF WATER RESO, OFF OF STATE ENG, CO CO003 - CITY OF COLORADO SPRINGS WATER DIVISION, CO CO007 - COLORADO STATE HEALTH DEPARTMENT, CO 
COOIO - COLORADO WATER RESOURCES RESEARCH INST, CO CO023 - DENVER REGIONAL COUNCIL OF GOVERNMENTS, CO COOOI - DENVER WATER DEPARTMENT, CO COO11 - INTRA SEARCH, INC, CO CO008 - METROPOLITAN DENVER SEWAGE DISPOSAL DIST \# 1 , CO CO024 - MSM CONSULTANTS, INC, CO CO015 - OCCIDENTAL OIL SHALE, INC, CO COOI2 - PIKES PEAK AREA COUNCIL OF GOVERNMENTS, CO COOO5 - PUEBLO BOARD OF WATER WORKS, CO COOI4 - PUEBLO REGIONAL PLANNING COMMISSION, CO COO22 - RIO BLANCO OIL SHALE COMPANY, CO COOO9 - ROCKY MOUNT ARSENAL, CO CO021 - W W WHEELER AND ASSOCIATES, INC, CO COOI7 - WESTERN ENGINEERS, INC, CO CO018 - WOODWARD-CLYDE CONSULTANTS, CO CO0I6 - WRIGHT WATER ENGINEERS, INC, CO

\section{CONNECTICUT}

CTO04 - BRIDGEPORT HYDRAULIC COMPANY, CT CTOO8 - CITY OF WEST HARTFORD PLANNING DEPT, CT CTO07 - CONNECTICUT DEPARTMENT OF TRANSPORTATION, CT CTO03 - CONNECTICUT DEPT OF ENVIRONMENTAL PROTECTION, CT CTOOI - ENVIR HEALTH SERVICE DIV, CT DEPT OF HEALTH, CT 
ORGANI -

ZATION

CODE

ORGANIZATION NAME

CT002 - METROPOLITAN (HARTFORD) DISTRICT WATER BUREAU,CT CTOO5 - UNIVERSITY OF CONNECTICUT, CT

\section{DELAWARE}

DE002 - DELAWARE DEPARTMENT OF NATURAL RESOURCES, DE DE005 - DELAWARE DEPARTMENT OF TRANSPORTATION, DE DEO0I - DELAWARE GEOLOGICAL SURVEY, DE DE004 - NEW CASTLE COUNTY WATER RESOURCES AGENCY, DE DE003 - WATER RESOURCES CENTER, UNIV OF DELAWARE, DE

\section{DISTRICT OF COLUMBIA}

DCO0I - DIST OF COLUMBIA DEPT OF ENVIR SERVICES, DC DC005 - DISTRICT OF COLUMBIA DEPT OF TRANSPORTATION, DC DC004 - DISTRICT OF COLUMBIA WATER RESO RESE CENTER, DC DCOO3 - WAPORA, INC, DC

\section{FLORIDA}

FLIO5 - BROMWELI ENGINEERING, INC, FL FLOOI - BUR OF GEOLOGY, FLORIDA DEPT OF NATURAL RESO, FL FL039 - CITY OE JACKSONVILLE WATER CONSERVATION, FL FL097 - CITY OF LAKE MARY WATER DEPARTMENT, FL FL043 - CITY OF PENSACOLA, FL 
FL103 - CNTR FOR COASTAL ZONE RESE,FL INST OF TECHNOL, FL FL016 - DADE COUNTY, FL FL106 - EDWARD E CLARK ENGINEERS-SCIENTISTS, INC, FL FLO02 - FLORIDA DEPARTMENT OF TRANSPORTATION, FL FLO5I - FLORIDA DEPT OF ENVIRONMENTAL REGULATION, FL FL107 - FLORIDA INSTITUTE OF PHOSPHATE RESEARCH, FL FLO96 - HILLSBOROUGH CNTY ENVIR PROTECTION COMM, FL FL095 - HILLSBOROUGH COUNTY HEALTH DEPARTMENT, FL FL099 - HUNTER AERIAL SURVEYS, FL FL022 - LEE COUNTY, FL FL060 - MANATEE COUNTY HEALTH DEPARTMENT, FL FL083 - NORTHWEST FLORIDA WATER MANAGEMENT DISTRICT, FL FL100 - PAN AMERICAN SURVEYS, INC, FL FL104 - REAL ESTATE DATA, INC, FL FLO06 - REEDY CREEK IMPROVEMENT DISTRICT, FL FL069 - SARASOTA COUNTY PUBLIC WORKS, FL FLO98 - SEABURN AND ROBERTSON, INC, FL FLIOI - SOUTH FLORIDA ENVIRON RESEARCH FOUNDATION, FL FL005 - SOUTH FLORIDA WATER MANAGEMENT DISTRICT, FL FL007 - SOUTHWEST FLORIDA WATER MANAGEMENT DISTRICT, FL FL085 - ST JOHNS RIVER WATER MANAGEMENT DISTRICT, FL FL084 - SUWANNEE RIVER WATER MANAGEMENT DISTRICT, FL FL030 - VOLUSIA COUNTY, FL 
FLO12 - WINTER HAVEN BOAT COURSE DISTRICT, FL

GEORGI A

GA008 - ATLANTA WATER WORKS, GA

GA022 - BRUNSWICK PULP LAND CO--WOODS DIVISION, GA

GA021 - CAMDEN COUNTY OFFICE OF TAX ASSESSOR, GA

GAO20 - CHATHAM CNTY-SAVANNAH METRO PLANNING COMM, GA

GA023 - CITY OF ATLANTA DEPT OF PUBLIC WORKS, GA

GA015 - CITY OF SAVANNAH INDUST \& DOMESTIC WA SUPPLY, GA

GAO10 - COLUMBUS WATER WORKS, GA

GAO09 - ENVIR PROT DIV, GEORGIA DEPT OF NATURAL RESO, GA

GA012 - GA DEPT NAT RESO, GEOLOGIC \& WA RESO DIV, GA

GA004 - GAINESVILLE SUPERINTENDENT OF WATER WORKS, GA

GAOI6 - GEORGIA DEPARTMENT OF TRANSPORTATION, GA

GA013 - GEORGIA INST OF TECHNOLOGY, ENVIR RESO CNTR, GA

GA006 - GRIFFIN SUPERINTENDENT OF WATER WORKS, GA

GA017 - LAW ENGINEERING TESTING COMPANY, GA

GA014 - LOWE ENGINEERS, INC, GA

GA007 - MACON-BIBB CNTY WATER \& SEWERAGE AUTHORITY, GA

GA018 - MARINE EXTENSION SERVICE, UNIV OF GEORGIA, GA

GAOI9 - MC INTOSH COUNTY OFFICE OF TAX ASSESSOR, GA

GA005 - ROME CITY MANAGER, GA

GAO01 - SAVANNAH DEPARTMENT OF WATER \& SEWAGE, GA 
GA011 - SOUTHWEST GEORGIA PLANNING \& DEVEL COMM, GA

GA002 - THOMASVILLE WATER \& LIGHT DEPARTMENT, GA

GA003 - VALDOSTA WATER \& SEWER DEPARTMENT, GA

\section{GUAM}

GQOII - DEPT OF PUBLIC WORKS, GOVT OF GUAM GQ008 - PUBLIC UTILITY AGENCY-WATER DIV, GOVT OF GUAM GQ009 - WATER RESOURCES RESE CENTER, UNIV OF GUAM

\section{HAWAI I}

HIOII - AIR SURVEY. HAWAII, HI

HIOO1 - BOARD OF WATER SUPPLY, CITY \& CNTY-HONOLULU, HI HI004 - BOARD OF WATER SUPPLY, COUNTY OF HAWAII, HI HI003 - BOARD OF WATER SUPPLY, COUNTY OF MAUI, HI HI002 - DEPT OF WATER, COUNTY OF KAUAI, HI HI006 - DIV-FISH \& GAME, HI DEPT OF LAND \& NAT RESO, HI HI007 - DIV-WATER \& LAND DEV, HI DEPT-LAND \& NAT RESO,HI HIOO5 - HAWAII DEPT OF HAWAIIAN HOME LANDS, HI HI008 - HAWAII DEPT OF TRANSPORTATION, HI HIOO9 - R M TOWILL CORPORATION, HI HIOIO - WATER RESOURCES RESE CNTR, UNIV OF HAWAII, HI 


\section{IDAHO}

IDO09 - ADA COUNTY PLANNING ASSOCIATION, ID

ID007 - AERIAL MAPPING COMPANY, ID

IDOII - IDAHO BUREAU OF MINES AND GEOLOGY, ID

ID004 - IDAHO DEPARTMENT OF HEALTH AND WELFARE, ID

IDOOI - IDAHO DEPARTMENT OF WATER RESOURCES, ID

IDO05 - IDAHO DEPT OF PUBLIC LANDS--TECH SERV SECT, ID

ID008 - IDAHO DEPT-TRANSPORTATION, DIV OF HIGHWAYS, ID

IDO02 - IDAHO FISH AND GAME DEPARTMENT, ID

ID006 - IDAHO REPROGRAPHICS, ID

IDOIO - IDAHO STATE COLLEGE, ID

IDOI3 - POTLATCH CORPORATION, TIMBERLANDS DEPT, ID

IDO03 - WATER RESOURCES RESEARCH INST, UNIV OF IDAHO, ID

IDOI2 - WESTERN WAYS, INC, ID

\section{ILLINOIS}

ILOI5 - ARGONNE NATIONAL LABORATORY, IL

IL0I6 - CHICAGO AERIAL SURVEY, IL

ILO05 - DEPAUL UNIVERSITY, IL

ILOOI - ILLINOIS DEPARTMENT OF PUBLIC HEALTH, IL

ILO04 - ILLINOIS DEPT OF TRANSPORTATION, IL

IL008 - ILLINOIS INSTITUTE OF NATURAL RESOURCES, IL 
ILOIO - ILLINOIS NATURAL HISTORY SURVEY, IL

IL003 - ILLINOIS STATE WATER SURVEY, IL

ILO02 - METRO SANITARY DIST OF GREATER CHICAGO, IL

IL013 - NALCO CHEMICAL COMPANY, IL

ILOI8 - NORTHEASTERN ILLINOIS PLANNING COMMISSION, IL

ILOI4 - RANDOLPH \& ASSOCIATES, INC, IL

ILOI7 - SARGENT \& LUNDY ENGINEERS, IL

ILOII - SIDWELL COMPANY, IL

ILO06 - STATE OF ILL ENVIRONMENTAL PROTECTION AGENCY, IL

ILOI2 - TECHNICAL PUBLISHING CO--POLLUT ENGINEERING, IL IL007 - WATER RESOURCES CENTER, UNIV OF ILLINOIS, IL

\section{INDI ANA}

IN007 - HOLCOMB RESEARCH INSTITUTE, IN

INO0I - IND ST BRD OF HEALTH, DIV STREAM POLL CONT, IN

INO02 - INDIANA DEPARTMENT OF NATURAL RESOURCES, IN

IN004 - INDIANA PLANNING \& DEVEL COM, REG 6, IN

INO05 - INDIANA STATE BOARD OF HEALTH, IN

IN008 - INDIANA STATE HIGHWAY COMMISSION, IN

INO06 - PURDUE UNIVERSITY, IN

I OWA

IA007 - AGRICULTURAL ENG DEPARTMENT, IOWA STATE UNIV, IA 
IA009 - COUNCIL BLUFFS WATER WORKS, IA

IAOI2 - DEPARTMENT OF CIVIL ENG, IOWA STATE UNIV, IA

IA005 - DEPARTMENT OF CIVIL ENGINEERING, UNIV OF IOWA, IA

IAOI 7 - DEPARTMENT OF EARTH SCIENCE, IOWA STATE UNIV, IA

IA013 - DEPARTMENT OF GEOGRAPHY, UNIV OF IOWA, IA

IA010 - DES MOINES COUNTY DRAINAGE DIST NO 7, IA

IA003 - DES MOINES WATER WORKS, IA

IA0I5 - ENGINEERING RESEARCH INSTITUTE, IA STATE UNIV, IA

IA008 - FORT DODGE DEPT OF MUNICIPAL UTILITIES, IA

IAOII - GREEN BAY LEVEE \& DRAINAGE DIST NO 2, IA

IAOI9 - IOWA DEPARTMENT OF ENVIRONMENTAL QUALITY, IA

IA020 - IOWA DEPARTMENT OF TRANSPORTATION, IA

IA006 - IOWA DEPT OF PREVENT MEDICINE \& ENVIR HEALTH, IA

IA018 - IOWA GEOLOGICAL SURVEY, IOWA CITY, IA

IAOI4 - IOWA INST OF HYDRAULIC RESEARCH, UNIV OF IOWA, IA

IA016 - IOWA STATE AGR EXP STATION, IA STATE UNIV, IA

IAO02 - LAKESIDE LABORATORY, UNIV OF IOWA, IA

IAO04 - OTTUMWA WATER WORKS, IA

IAOOI - UNIVERSITY HYGIENIC LABORATORY, IA

\section{KANSAS}

KS003 - DIV OF WATER RESO, KANSAS STATE BRD OF AGR, KS KSOOI - ENVIR HEALTH SERVICE, KS STATE DEPT OF HEALTH, KS 
ORGANI -

ZATION

CODE

ORGANI ZATION NAME

KSO02 - KANSAS CITY BOARD OF PUBLIC UTILITIES, KS

KSO06 - KANSAS DEPARTMENT OF TRANSPORTATION, KS

KSO05 - KANSAS FISH \& GAME COMMISSION, KS

KSO04 - TOPEKA WATER DEPARTMENT, KS

\section{KENTUCKY}

KY003 - DIV-SANIT ENG, KY DEPT FOR HUMAN RESOURCES, KY KYOII - GRW AERIAL SURVEY, INC, KY

KYOO9 - KENTUCKY DEPARTMENT OF TRANSPORTATION, KY

KYOOI - KENTUCKY DEPT FOR NAT RESO \& ENVIR PROT, KY

KYO05 - KENTUCKY DEPT OF FISH \& WILDLIFE, KY

KYOO2 - KENTUCKY GEOLOGICAL SURVEY, UNIV OF KENTUCKY, KY KY004 - LOUISVILLE WATER COMPANY, KY

KYOIO - PARK AERIAL SURVEYS, KY

KY007 - WATER RESOURCES LAB, UNIV OF LOUISVILLE, KY YVDO8 - WATER RESOURCES RESE INST, UNIV OF KENTUCKY, KY

LOUI SI ANA

LA008 - BOSSIER CITY WATER PLANT, LA

LAO12 - CENTER FOR WETLAND RESOURCES (LSU), LA

LAOIO - DIV-WATER POLL CONT, LA WILDLIFE \& FISH COMM, LA

LAONG - EAST JEFFERSON WATER WORKS DISTRICT NO I, LA 
ORGANI -

ZATION

CODE

ORGANIZATION NAME

LA003 - HOUMA WATER TREATMENT PLANT, LA

LAO04 - JEFFERSON WATER WORKS DISTRICT NO 2, LA

LAO05 - LAFOURCHE WATER WORKS DISTRICT NO 1, LA

LAOI4 - LOUISIANA DEPT OF TRANSPORT \& DEVELOPMENT, LA

LAOI3 - LOUISIANA DEPT OF WILDLIFE AND FISHERIES, LA

LA002 - LOUISIANA STATE DEPARTMENT OF HEALTH, LA

LAOO9 - MONROE UTILITIES COMM WATER TREATMENT PLANT, LA

LA007 - NEW ORLEANS SEWERAGE \& WATER BOARD, LA

LAOOI - RAPIDES PARISH WATER WORKS DISTRICT NO 3, LA

LAOII - SHREVEPORT DEPARTMENT OF WATER \& SEWERAGE, LA

\section{MAINE}

ME004 - MAINE DEPARTMENT OF HUMAN SERVICES, ME ME008 - MAINE DEPARTMENT OF TRANSPORTATION, ME MEOO1 - MAINE DEPT OF ENVIRONMENTAL PROTECTION, ME ME005 - MAINE DEPT OF INLAND FISHERIES AND WILDLIFE, ME ME007 - MAINE DEPT OF MARINE RESOURCES, ME ME002 - MAINE GEOLOGICAL SURVEY, ME ME003 - MAINE STATE PLANNING OFF, RESO PLANNING DIV, ME ME006 - UNIVERSITY OF MAINE, ME

MARYLAND

MDO12 - AEROECO, MD 


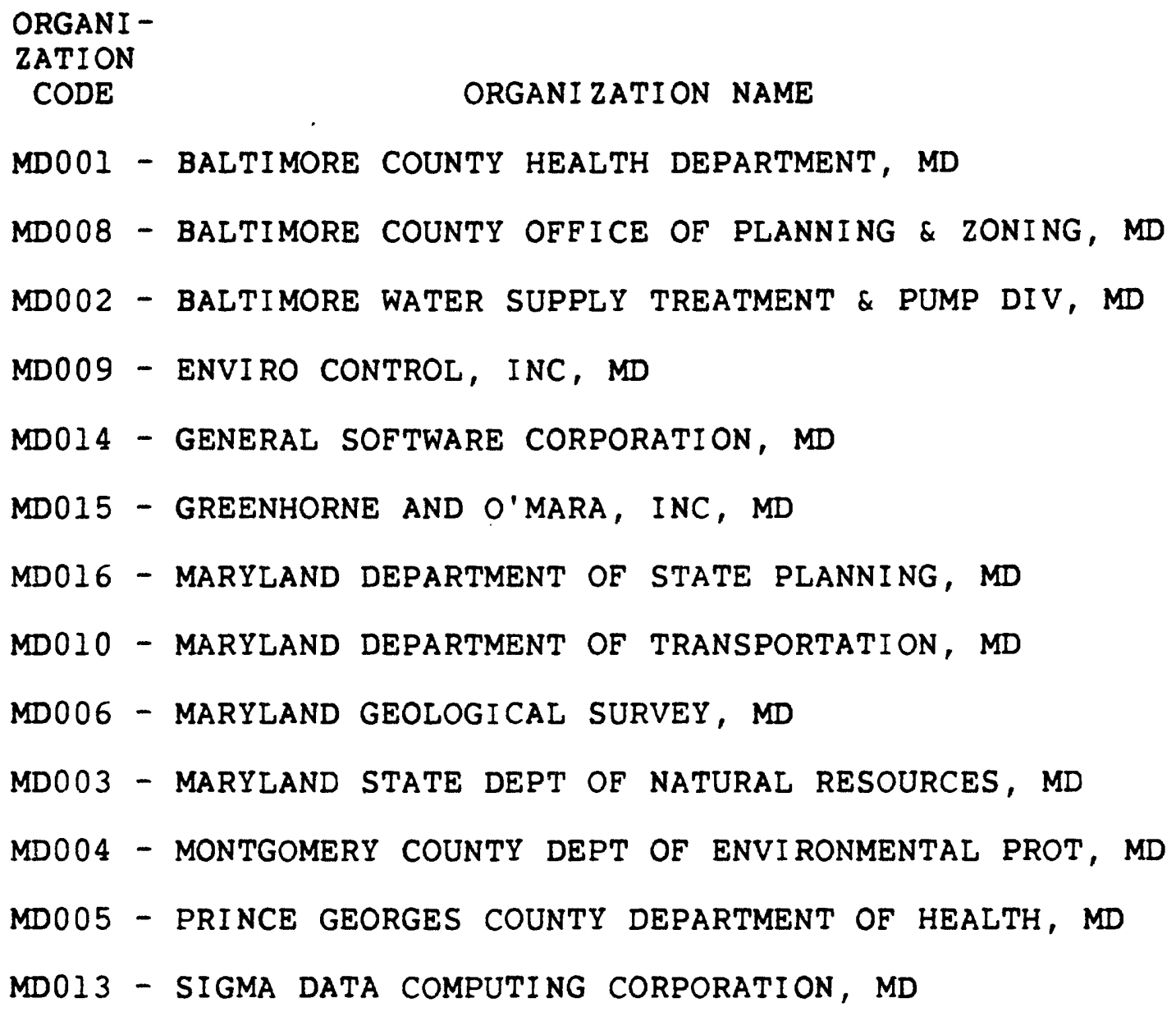

\section{MASSACHUSETTS}

MA009 - AVIS AIRMAP, INC, MA

MA007 - BARNSTABLE COUNTY HEALTH DEPARTMENT, MA

MAOII - BOSTON REDEVELOPMENT AUTHORITY, MA

MA001 - GIDLEY LABORATORIES, INC, MA

MAO08 - MASSACHUSETTS DEPARTMENT OF PUBLIC WORKS, MA

MA003 - MASSACHUSETTS DIV OF WATER POLLUTION CONTROL, MA

MAOIO - MASSACHUSETTS WATER RESOURCES COMMISSION, MA

MA006 - MILLIPORE CORPORATION, MA 
ORGANI -

ZATION

CODE

ORGANIZATION NAME

MAO05 - THERMO-ELECTRIC CORPORATION, MA

MA002 - WATER RESOURCES RESEARCH CNTR, UNIV OF MASS, MA MA004 - WILLIAM T LORENZ \& COMPANY, MA

\section{CHIGAN}

MI007 - ABRAMS AERIAL SURVEY CORPORATION, MI

MI008 - BENDIX AEROSPACE SYSTEMS DIVISION, MI

MI009 - CAPITOL AIR SURVEY, MI

MIOI0 - ENVIRONMENTAL RESEARCH INSTITUTE OF MICHIGAN, MI

MIO06 - JOHNSON \& ANDERSON, INC, MI

MIOII - MICH DEPT OF STATE HIGHWAYS \& TRANSPORTATION, MI

MIOOI - MICHIGAN DEPARTMENT OF NATURAL RESOURCES, MI

MIO04 - MICHIGAN GRAND RIVER WATERSHED, MI

MI002 - MICHIGAN STATE UNIVERSITY, MI

MIO03 - NATIONAL SANITATION FOUNDATION, MI

MIO05 - NORTHWEST WATERSHED PROJECT, MI

MI013 - S CENTRAL MICH PLAN \& DEVEL COUNCIL-REG III, MI

MIOI4 - SOUTHEAST MICHIGAN COUNCIL OF GOVERNMENTS, MI

MIOI5 - STEREO FOTO, INC, MI

MIOI6 - WEST MICHIGAN REGIONAL PLANNING COMM, MI

MINNESOTA

MNOO9 - BLANDIN PAPER COMPANY, MN 


\section{ORGANI -}

ZATION

CODE

ORGANI ZATION NAME

MN007 - DULUTH WATER, GAS \& SEWAGE TREATMENT DEPT, MN MNO02 - EVELETH TACONITE COMPANY, MN

MNOOI - HENNEPIN COUNTY HIGHWAY DEPARTMENT, MN

MNO15 - MARK HURD AERIAL SURVEYS, INC, MN

MNO20 - MARTINEZ MAPPING AND ENGINEERING, INC, MN

MNO21 - METROPOLITAN COUNCL OF THE TWIN CITIES AREA, MN

MNOII - METROPOLITAN (ST PAUL) WASTE CONTROL COMM, MN

MNOO3 - MINNESOTA DEPT OF NATURAL RESOURCES, MN

MNO18 - MINNESOTA DEPT OF TRANSPORTATION, MN

MNOI9 - MINNESOTA HEALTH DEPARTMENT, MN

MNOO8 - MINNESOTA ORE OPER, USS CORP, MN

MNO12 - MINNESOTA POLLUTION CONTROL AGENCY, MN

MNOIO - MINNESOTA POWER \& LIGHT COMPANY, MN

MN016 - MINNESOTA STATE PLANNING AGENCY, MN

MNO06 - NORTHERN STATE POWER COMPANY, MN

MNO04 - OTTER TAIL POWER COMPANY, MN

MNO05 - RAMSEY COUNTY ENVIRONMENTAL SERVICE, MN

MNOI4 - UNITED POWER ASSOCIATION, MN

MNO13 - WASHINGTON COUNTY HIGHWAY DEPARTMENT, MN

MNO17 - WATER RESOURCES RESEARCH CTR, UNIV OF MINN, MN

\section{SSI SSI PPI}

MS005 - CITY OF COLUMBUS LIGHT \& WATER DEPARTMENT, MS 


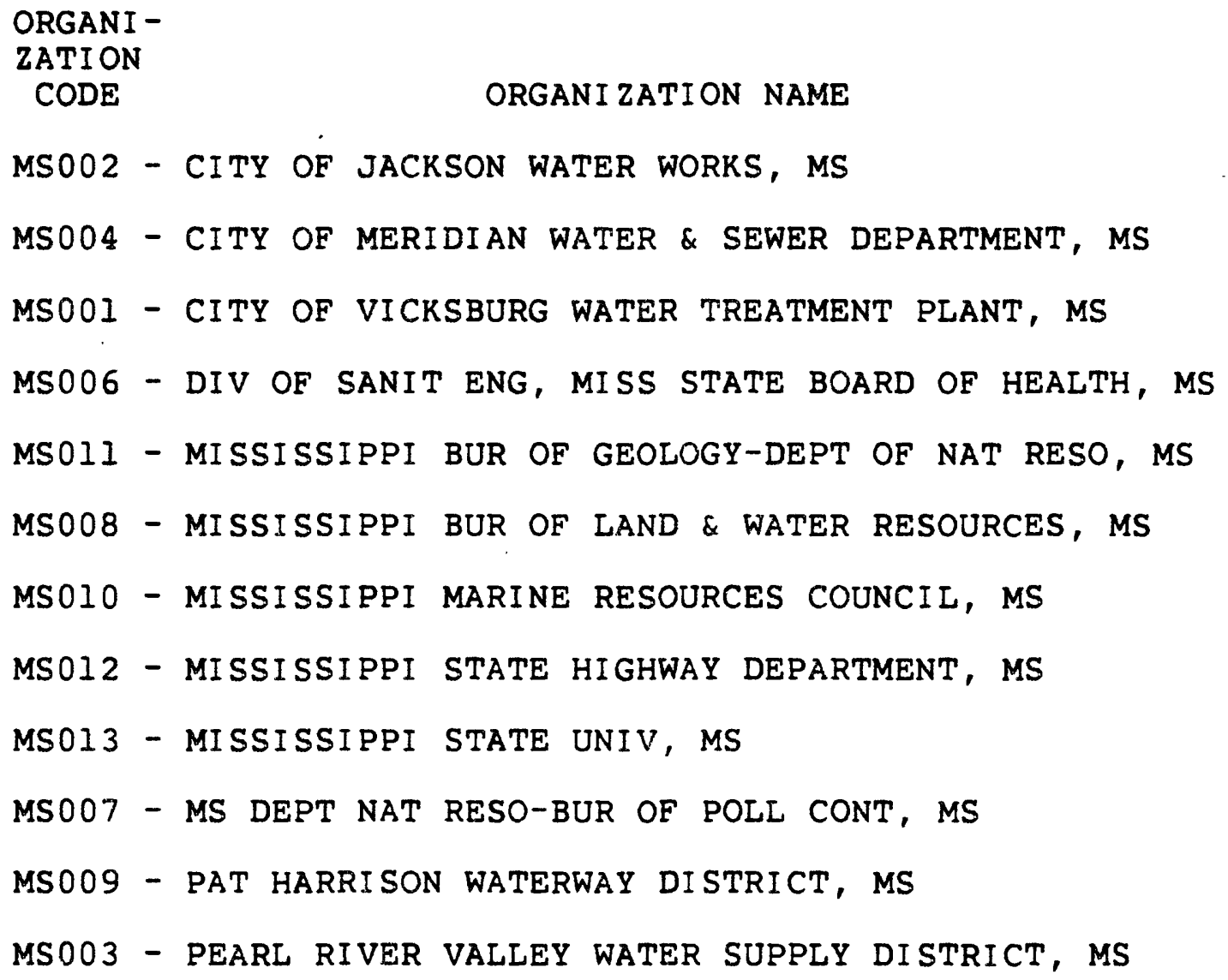

MI SSOURI

MOOI4 - BLACK \& VEATCH CONSULTING ENGINEERS, MO

MOOII - BURNS AND MC DONNELL, MO

MO004 - LITTLE RIVER DRAINAGE DISTRICT, MO

MO003 - METROPOLITAN ST LOUIS SEWER DISTRICT, MO

MOOIO - MID-AMERICA REGIONAL COUNCIL, MO

MOOOI - MISSOURI DIVISION OF HEALTH, MO

MOOI2 - MISSOURI STATE HIGHWAY DEPARTMENT, MO

MO006 - MISSOURI WATER POLLUTION CONTROL PROGRAM, MO

MOO05 - MO DIV OF GEOL \& LAND SURVEY, DEPT-NAT RESO, MO 
ORGANI -

ZATION

CODE

ORGANI ZATION NAME

MO009 - SURDEX CORPORATION, MO

MO013 - UNICORN FEASIBILITY, MO

MO007 - UNION ELECTRIC COMPANY, MO

MO002 - UNIVERSITY OF MISSOURI, MO

MO008 - WATER RESOURCES RESE CNTR, UNIV OF MISSOURI, MO

\section{MONTANA}

MTO05 - MONTANA BUREAU OF MINES \& GEOLOGY, MT

MTO03 - MONTANA DEPARTMENT OF HEALTH \& ENVIR SCIENCES,MT

MTO09 - MONTANA DEPARTMENT OF HIGHWAYS, MT

MTO01 - MONTANA DEPT OF FISH, WILDLIFE \& PARKS, MT

MTOO4 - MONTANA DEPT OF NATURAL RESO \& CONSERVATION, MT

MTO08 - MONTANA ENERGY \& MHD RESE \& DEVELOPMENT INST, MT

MTO02 - MONTANA UNIV JOINT WATER RESOURCES RESE CNTR, MT

MT007 - UNIVERSITY OF MONTANA, MT

MT006 - WESTECH, INC, MT

NEBRASKA

NEOII - HOSKINS-WESTERN-SONDEREGGER, INC, NE

NEO05 - NEBRASKA DEPARTMENT OF HEALTH, NE

NE009 - NEBRASKA DEPARTMENT OF ROADS, NE

NEO10 - NEBRASKA DEPARTMENT OF WATER RESOURCES, NE 
ORGANI -

ZATION

CODE

ORGANIZATION NAME

NE00I - NEBRASKA GAME \& PARKS COMMISSION, NE

NE006 - NEBRASKA NATURAL RESOURCES COMMISSION, NE

NE003 - OMAHA METROPOLITAN UTILITIES DISTRICT, NE

NEO04 - SOIL \& WATER TESTING LAB, UNIV OF NEBRASKA, NE

NE008 - UNIV OF NEBRASKA, CONSERVATION \& SURVEY DIV, NE

NEO02 - WATER POLL CONT DIV, NEBR DEPT OF ENVIR CONT, NE

NE007 - WATER RESOURCES CENTER, UNIV OF NEBRASKA, NE

\section{NEVADA}

NV026 - CARSON CITY DEPT OF PUBLIC WORKS, NV

NV02I - CARSON RIVER BASIN COUNCIL OF GOVERNMENTS, NV

NV0I5 - CLARK COUNTY HEALTH DISTRICT, NV

NVOI4 - COLLEGE OF AGRICULTURE, UNIV OF NEVADA, NV

NV013 - DESERT RESEARCH INSTITUTE, UNIV OF NEVADA, NV

NV003 - DIV-WATER RESO, NEVADA DEPT-CONS \& NAT RESO, NV

NV005 - DIV OF RENEWABLE NAT RESO, UNIV OF NEVADA, NV

NV029 - GREAT BASIN AERIAI SURVEYS, NV

NV028 - KAMINSKI STUDIOS LTD, NV

NVOI7 - LAS VEGAS VALLEY WATER DISTRICT, NV

NVOO7 - NEVADA BUREAU OF MINES \& GEOLOGY, NV

NVOI2 - NEVADA CONSUMER HEALTH PROTECTION SERVICE, NV

NVOO9 - NEVADA DEPARTMENT OF FISH \& GAME, NV

NVOOI - NEVADA DIVISION OF ENVIRONMENTAL PROTECTION, NV 
ORGANI -

ZATION

CODE

ORGANIZATION NAME

NVOIO - NEVADA DIVISION OF FORESTRY, NV

NVOII - NEVADA DIVISION OF PARKS, NV

NV006 - NEVADA STATE BUREAU OF ENVIRONMENTAL HEALTH, NV

NVOO8 - NEVADA STATE HIGHWAY DEPARTMENT, NV

NVO25 - RENO-SPARKS JOINT WASTE WAT POLLUT CONT PLANT,NV

NV004 - ROUNDHILI GENERAL IMPROVEMENT DISTRICT, NV

NVO18 - SIERRA PACIFIC POWER COMPANY, NV

NV027 - UPSON-DONDERO STUDIOS, NV

NVOO2 - WALKER RIVER IRRIGATION DISTRICT, NV

NV023 - WALTERS ENGINEERING, INC, NV

NV020 - WASHOE COUNCIL OF GOVERNMENTS, NV

NV016 - WASHOE COUNTY DISTRICT HEALTH DEPARTMENT, NV

NEW HAMPSHIRE

NHOO4 - BCI GEONETICS, INC, NH

NHOO3 - NEW HAMPSHIRE DEPT OF PUB WKS AND HIGHWAYS, NH

NHOO2 - NEW HAMPSHIRE WAT SUPPLY \& POLLUT CONTROL COM,NH

NHOOI - WATER RESO RESE CNTR, UNIV OF NEW HAMPSHIRE, NH

NEW JERSEY

NJ013 - AERIAL DATA REDUCTION ASSOC, NJ

NJ016 - ATLANTIC COUNTY DIVISION OF PLANNING, NJ 
NJ019 - BERGEN COUNTY PLANNING BOARD, NJ

NJ012 - CAPE MAY COUNTY OF PLANNING BOARD, NJ

NJ017 - CUMBERLAND COUNTY PLANNING BOARD, NJ

NJO02 - DIV-WATER RESO, NJ DEPT OF ENVIR PROTECTION, NJ

NJOII - GEOD AERIAL SURVEYS, INC, NJ

NJ020 - HUNTERDON COUNTY PLANNING BOARD, NJ

NJ005 - KEARNEY (TOWN OF) WASTEWATER TREATMENT PLANT, NJ

NJ021 - MERCER COUNTY PLANNING BOARD, NJ

NJ015 - MIDLANTIC AERIAL SURVEYS, NJ

NJ022 - MIDDLESEX COUNTY PLANNING BOARD, NJ

NJ023 - MONMOUTH COUNTY PLANNING BOARD, NJ

NJ008 - MONTCLAIR STATE COLLEGE, NJ

NJO24 - MORRIS COUNTY PLANNING BOARD, NJ

NJ007 - NEW JERSEY BUR OF GEOLOGY \& TOPOGRAPHY, NJ

NJO10 - NEW JERSEY DEPARTMENT OF TRANSPORTATION, NJ

NJOI4 - OCEAN COUNTY PLANNING BOARD, NJ

NJ004 - PASSAIC COUNTY, NJ

NJ001 - PASSAIC VALLEY WATER COMMISSION, NJ

NJO09 - ROBINSON AERIAL SURVEYS, INC, NJ

NJ018 - SALEM COUNTY PLANNING BOARD, NJ

NJ025 - SOMERSET COUNTY PLANNING BOARD, NJ

NJ026 - UNION COUNTY DIV OF PLANNING \& DEVEL, NJ

NJ003 - WANAQUE LAB, NORTH JERSEY DIST WATER SUP COM, NJ 
NJ006 - WATER RESOURCES RESEARCH INST, RUTGERS UNIV, NJ

\title{
NEW MEXICO
}

NM005 - LOS ALAMOS SCIENTIFIC LABORATORY, NM NMO02 - N MEX STATE HEALTH \& ENVIRONMENT DEPT, NM NM003 - NEW MEXICO INST OF MINING AND TECHNOLOGY, NM NMOO1 - NEW MEXICO STATE ENGINEERS OFFICE, NM NM007 - NEW MEXICO STATE HIGHWAY DEPARTMENT, NM NMO04 - NEW MEXICO STATE UNIVERSITY, NM NM006 - THOMAS R MANN AND ASSOCIATES, NM

\author{
NEW YORK
}

NY018 - AERO SERVICE CORPORATION, NY

NY005 - AEROGRAPHICS, INC, NY

NYO14 - BROOKHAVEN NATIONAL LABORATORY, NY

NY032 - CITY OF ALBANY PLANNING DEPT, NY

NY006 - CITY OF ROCHESTER WATER TREATMENT PLANT, NY

NY013 - CORNELL UNIVERSITY, NY

NYOO2 - DIV-SANIT \& WATER SUP, NASSAU CNTY PUB SUP, NY NY025 - DONALD A THOMAS, LAND SURVEYOR (GENESEO), NY NY029 - EL COMP DATA SYSTEMS, INC, NY NY020 - GEO MAP, INC, NY 
NY0I5 - LOCKWOOD, KESSLER \& BARTLETT, INC, NY NYOO9 - MONROE COUNTY WATER AUTHORITY, NY NY007 - NEW YORK CITY DEPT OF ENVIR PROTECTION, NY NY017 - NEW YORK PUBLIC INTEREST RESEARCH GROUP, NY NYO22 - NEW YORK STATE DEPARTMENT OF HEALTH, NY NYO28 - NEW YORK STATE ELECTRIC \& GAS CORPORATION, NY NY012 - NY STATE UNIV AT BUFFALO, GREAT LAKES LAB, NY NY016 - NYC BUR OF WATER POLLUTION CONTROL, NY NYOOI - NEW YORK STATE DEPT OF ENVIRONMENTAL CONS, NY NY021 - NY STATE EDUCATION DEPARTMENT, NY NY030 - O'BRIEN AND GERE ENGINEERS, INC, NY NYO10 - ONONDAGA COUNTY DEPT OF DRAINAGE \& SANIT, NY NYOII - RENNSELAER POLYTECHNIC INSTITUTE, NY NY019 - SANBORN MAP COMPANY, INC, NY NY031 - SCHENECTADY COUNTY PLANNING DEPT, NY NYOO3 - THE CARBORUNDUM COMPANY, NY NY004 - TRI-STATE REGIONAL PLANNING COMMISSION, NY NYOO8 - WESTCHESTER COUNTY DEPT OF PUBLIC WORKS, NY

NORTH CAROLINA

NC008 - CAROLINA POWER \& LIGHT COMPANY, NC NCOIO - CHARLES T MAIN, INC, NC NCO13 - CITY OF GREENSBORO DEPT OF PUBLIC WORKS, NC 
NCO05 - DUKE UNIV, SCHOOL OF ENG, DEPT OF CIVIL ENG, NC NCO04 - NC DEPT-NAT RESO \& COMMUNITY DEVELOPMENT, NC NC009 - NC UNIV, DEPT OF ENV SCIENCE \& ENG, NC NCO11 - NORTH CAROLINA DEPT OF TRANSPORTATION, NC NC007 - PIEDMONT AERIAL SURVEYS, INC, NC NC006 - SOUTHERN WTR RESO SCIENTIFIC INFO CNTR, NCU, NC NC003 - WATER SUPPLY BR, SES, DHS, NC DEPT HUMAN RESO, NC NCO12 - YONTS AND ASSOCIATES, NC

NORTH DAKOTA

ND004 - CITY OF BISMARCK WATER DEPARTMENT, ND ND005 - CITY OF DICKINSON WATER TREATMENT PLANT, ND ND006 - GRAND FORKS WATER TREATMENT PLANT, ND ND003 - MINOT CITY WATER TREATMENT PLANT, ND NDOOI - NORTH DAKOTA GAME \& FISH DEPARTMENT, ND NDO07 - NORTH DAKOTA GEOLOGICAL SURVEY, ND ND002 - NORTH DAKOTA STATE DEPARTMENT OF HEALTH, ND NDO11 - NORTH DAKOTA STATE HIGHWAY DEPARTMENT, ND NDOO9 - NORTH DAKOTA STATE WATER COMMISSION, ND NDO10 - WATER RESOURCES RESEARCH INST, ND STATE UNIV, ND

OHIO

OHOO9 - AERIAL ENG SECT, OHIO DEPT-TRANSPORTATION, OH 
OH018 - CITY OF CINCINNATI PLANNING DEPT, OH OHOO5 - CITY OF CLEVELAND QUALITY WATER DEPARTMENT, OH OH016 - COLE-LAYER-TRUMBLE, INC, OH OHO19 - CUYAHOGA COUNTY PLANNING DEPT, OH OH006 - DIV OF HIGHWAYS, OHIO DEPT OF TRANSPORTATION, OH OHO08 - GEAUGA COUNTY OFFICE OF SANITARY ENGINEER, OH $\mathrm{OHOI2} \mathrm{-} \mathrm{GRAPHCO,} \mathrm{OH}$ OHO13 - HEIDELBERG COLLEGE, OH OHOI7 - KUCERA \& ASSOCIATES, OH OHOO2 - MIAMI CONSERVANCY DISTRICT, $\mathrm{OH}$ OHOII - OHIO AGRICULTURAL RESE \& DEVELOPMENT CENTER, OH OHOO4 - OHIO ENVIRONMENTAL PROTECTION AGENCY, OH OHOI 4 - OHIO STATE UNIV CENTER FOR LAKES, OH OHOIO - OHIO STATE UNIVERSITY, DEPT OF AGRONOMY, OH OHO15 - OHIO DEPT OF NATURAL RESOURCES, OH OH007 - WATER RESOURCES CENTER, OHIO STATE UNIV, OH

\section{OKLAHOMA}

OKOO3 - OKLAHOMA CLIMATOLOGICAL SURVEY, OK OKOO4 - OKLAHOMA DEPARTMENT OF TRANSPORTATION, OK OKOOI - OKLAHOMA STATE DEPARTMENT OF HEALTH, OK OKO02 - OKLAHOMA WATER RESOURCES BOARD, OK 


\section{OREGON}

ORO12 - AERIAL MAPPING COMPANY OF OREGON, OR OR020 - BEND MAPPING AND BLUEPRINTING, INC, OR OR018 - CENTRAL BLUEPRINT COMPANY, AERO GRAPHICS DIV, OR OROI7 - CHICKERING GREEN EMPIRE, INC, OR ORO10 - DELANO PHOTOGRAPHICS, INC, OR OROO1 - DEPARTMENT OF FOREST ENG, OREGON STATE UNIV, OR OR003 - DOUGLAS COUNTY WATER RESOURCES SURVEY, OR OR016 - OREGON DEPT OF GEOLOGY \& MINERALS INDUSTRIES, OR OROO9 - OREGON STATE DEPT OF FISH AND WILDLIFE, OR OROO7 - OREGON STATE FORESTRY DEPARTMENT, OR OROI5 - OREGON STATE HIGHWAY DIVISION, OR OROO4 - OREGON WATER RESOURCES DEPARTMENT, OR OR014 - PORTLAND DEPARTMENT OF UTILITIES, OR ORO06 - PORTLAND GENERAL ELECTRIC COMPANY, OR ORO19 - SKYVIEW AERIAL SURVEYS, INC, OR OROII - SPENCER B GROSS--AERIAL PHOTOGRAPHY, OR OROO8 - THE CITY OF PENDLETON, OR ORO13 - WAC CORPORATION, OR

\section{PENNSYLVANIA}

PAO07 - ALLEGHENY COUNTY HEALTH DEPARTMENT, PA 


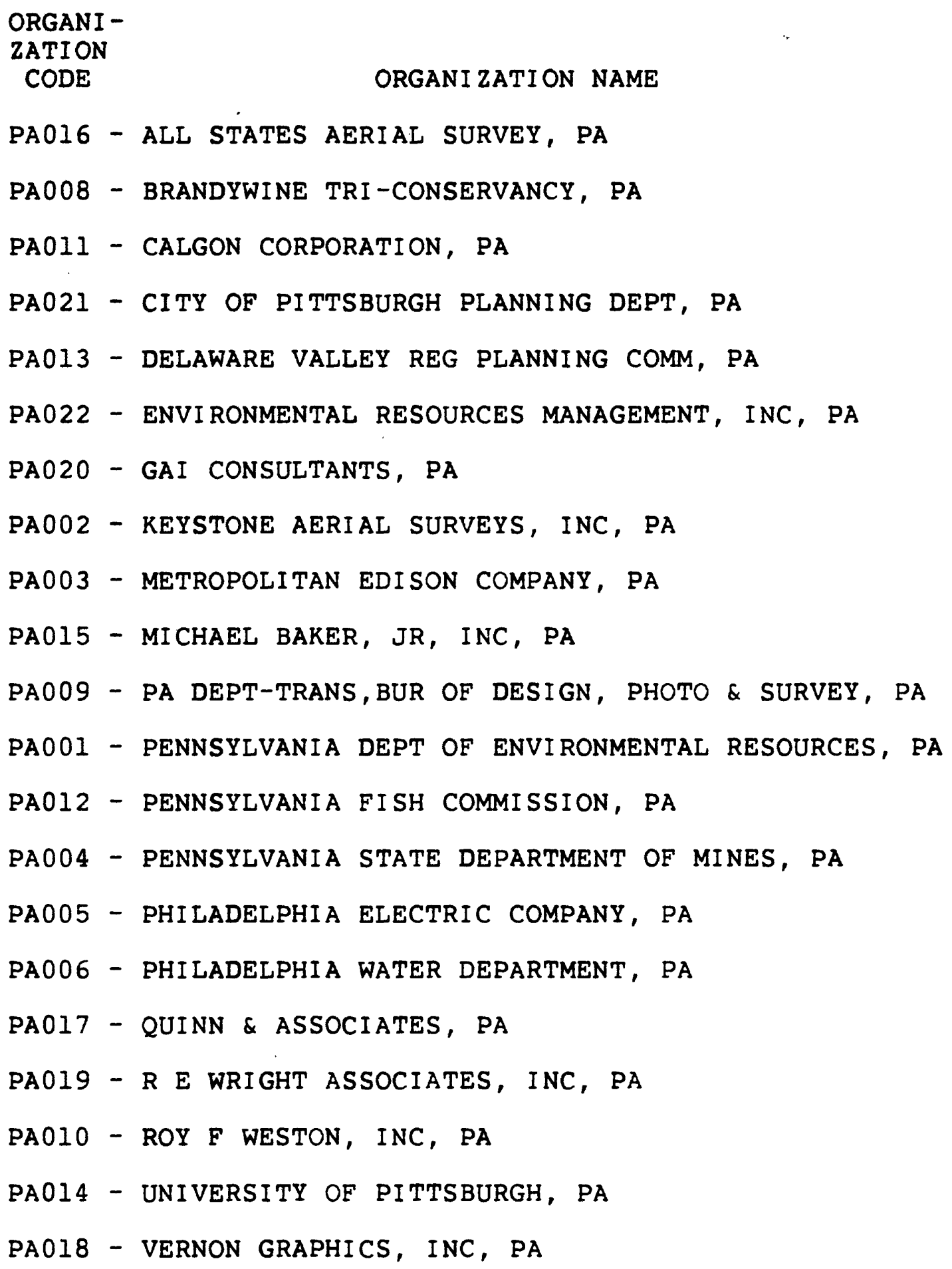


RQ005 - PUERTO RICO DEPARTMENT OF AGRICULTURE, PR

RQ003 - PUERTO RICO DEPARTMENT OF HEALTH, PR

RQ008 - PUERTO RICO DEPT OF NATURAL RESOURCES, PR

RQ006 - PUERTO RICO DEPT OF TRANSPORT \& PUBLIC WKS, PR

RQ004 - PUERTO RICO ENVIRONMENTAL QUALITY BOARD, PR

RQOOI - PUERTO RICO WATER RESOURCES AUTHORITY, PR

RQ002 - WATER RESO RESE INST, UNIV OF PUERTO RICO, PR

RHODE ISLAND

RIO02 - RHODE ISLAND DEPT OF ENVIR MANAGEMENT, RI

RI003 - RHODE ISLAND DEPT OF TRANSPORTATION, RI

RIOOI - UNIVERSITY OF RHODE ISLAND, RI

\section{SOUTH CAROLINA}

SC035 - ABBEVILLE COUNTY TAX ASSESSOR, SC

SCOOI - AGRICULTURAL ENG DEPARTMENT, CLEMSON UNIV, SC

SC029 - AIKEN COUNTY TAX ASSESSOR, SC

SC034 - ALLENDALE COUNTY TAX ASSESSOR, SC

SC037 - ANDERSON COUNTY TAX ASSESSOR, SC

SC030 - BAMBERG COUNTY TAX ASSESSOR, SC

SC027 - BARNWELL COUNTY TAX ASSESSOR, SC

SC054 - BEAUFORT COUNTY TAX ASSESSOR, SC 


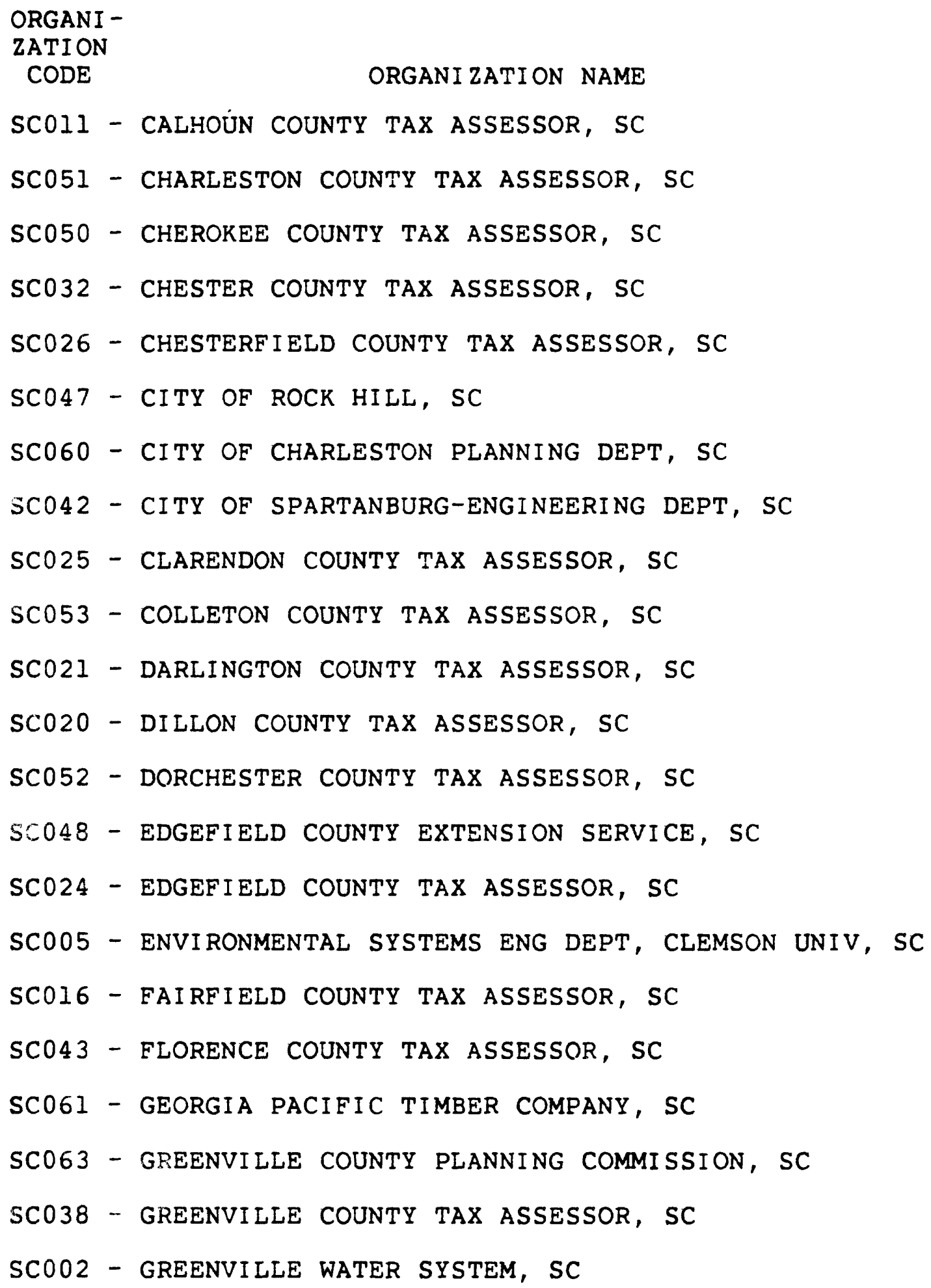


SCO3I - GREENWOOD COUNTY TAX ASSESSOR, SC

SC023 - HAMPTON COUNTY TAX ASSESSOR, SC

SC056 - HARRY COUNTY TAX ASSESSOR, SC

SC058 - JASPER COUNTY TAX ASSESSOR, SC

SCO09 - KERSHAW COUNTY TAX ASSESSOR, SC

SC033 - LANCASTER COUNTY TAX ASSESSOR, SC

SC039 - LAURENS COUNTY TAX ASSESSOR, SC

SCO17 - LEE COUNTY TAX ASSESSOR, SC

SCO14 - LEXINGTON COUNTY TAX ASSESSOR, SC

SC022 - MARION COUNTY TAX ASSESSOR, SC

SCO19 - MARLBORO COUNTY TAX ASSESSOR, SC

SCO18 - NEWBERRY COUNTY TAX ASSESSOR, SC

SC046 - OCONEE COUNTY TAX ASSESSOR, SC

SCO10 - ORANGEBURG COUNTY TAX ASSESSOR, SC

SC036 - PICKENS COUNTY TAX ASSESSOR, SC

SCO15 - RICHLAND COUNTY TAX ASSESSOR, SC

SCO13 - SALUDA COUNTY TAX ASSESSOR, SC

SC028 - SANTEE-COOPER PUBLIC SERVICE AUTHORITY, SC

SC007 - SC LAND RESOURCES CONSERVATION COMMISSION, SC

SC062 - SC WILDLIFE \& MARINE RESOURCES DEPT, SC

SC055 - SOUTH CAROLINA COASTAL COUNCIL, SC

SCOO4 - SOUTH CAROLINA DEPT OF HEALTH \& ENVIR CONTROL,SC SC045 - SOUTH CAROLINA FORESTRY COMMISSION, SC 
SC006 - SOUTH CAROLINA STATE HIGHWAY DEPARTMENT, SC SC008 - SOUTH CAROLINA WATER RESOURCES COMMISSION, SC SCO44 - SPARTANBURG COUNTY PLANNING COMMISSION, SC SC04I - SPARTANBURG COUNTY TAX ASSESSOR, SC SC003 - SPARTANBURG WATER WORKS, SC SCO12 - SUMTER COUNTY TAX ASSESSOR, SC SCO40 - UNION COUNTY TAX ASSESSOR, SC SC049 - WILLIAMSBURG COUNTY TAX ASSESSOR, SC SC059 - WACCAMAW REGIONAL PLANNING COUNCIL, SC

\section{SOUTH DAKOTA}

SDO04 - DAKOTA ENGINEERING COMPANY, SD

SDO02 - EAST DAKOTA CONSERVANCY SUBDISTRICT, SD

SD010 - SOIL TESTING LAB, SOUTH DAKOTA STATE UNIV, SD

- SDOI3 - SOUTH DAKOTA DEPARTMENT OF TRANSPORTATION, SD SDO08 - SOUTH DAKOTA DEPT OF ENVIRONMENTAL PROT, SD SDO05 - SOUTH DAKOTA DEPT WATER \& NAT RESOURCES, SD SDO03 - SOUTH DAKOTA GEOLOGICAL SURVEY, SD SDOO9 - SOUTH DAKOTA SCHOOL OF MINES AND TECHNOLOGY, SD SDOII - SOUTH DAKOTA STATE CHEMIST, UNIV OF S DAK, SD SDO12 - STATION BIOCHEMISTRY, S DAK STATE UNIV, SD SDOOI - WATER RESO RESEARCH INST, S DAK STATE UNIV, SD 
TENNESSEE

TNO05 - BRISTOL WATER PLANT, TN

TNOO4 - CITY WATER COMPANY OF CHATTANOOGA, TN

TNO03 - CLEVELAND UTILITIES WATER DIVISION, TN

TN010 - CONTINENTAL AERIAL SURVEYS, INC, TN

TN006 - DEPARTMENT OF AGR ENG, UNIV OF TENN EXPER STA,TN TN002 - DIV-WATER QUAL CONT, TN DEPT OF PUB HEALTH, TN TN007 - MEMPHIS LIGHT, GAS \& WATER DIVISION, TN TNOI1 - OAK RIDGE NATIONAL LABORATORY, TN TNOO9 - TENN TECH UNIV-ENVIR BIOLOGY RESE CENTER, TN TN013 - TENNESSEE DEPARTMENT OF CONSERVATION, TN TN012 - TENNESSEE DEPARTMENT OF TRANSPORTATION, TN TNOOI - TENNESSEE WILDLIFE RESOURCES AGENCY, TN TNO08 - WATER RESO REASEARCH CENTER, UNIV OF TENN, TN

TEXAS

TX030 - ADAMS AERIAL SURVEYS, TX

TXO29 - AERO COLOR, TX

TXO23 - AERO SERVICE CORPORATION, TX

TX008 - BUR OF ECONOMIC GEOLOGY (UNIV-TX AT AUSTIN), TX TX016 - CITY OF AUSTIN, ENGINEERING DEPARTMENT, TX TX017 - ESPEY, HUSTON \& ASSOCIATES, INC, TX 
TX019 - GRETEMAN AERIAL PHOTOMAPS, TX

TXO24 - HOUSTON LIGHTING AND POWER COMPANY, TX

TX04I - HOUSTON PUBLIC LIBRARY, TX

TX020 - INTERNATIONAL AERIAL MAPPING COMPANY, TX

TX035 - JERRY FOSTER \& COMPANY, TX

TX039 - K. M. NG \& ASSOCIATES, TX

TX036 - LANDIS AERIAL PHOTO, TX

TX037 - MAPSCO, TX

TX032 - METROPOLITAN AERIAL SURVEYS, TX

TX038 - MPSI MAPS, INC, TX

TX033 - NORTHERN TECHNICAL SERVICES, TX

TX026 - PETROLEUM INFORMATION CORPORATION, TX

TXO28 - RADIAN CORPORATION, TX

TXCO9 - RAILROAD COMMISSION OF TEXAS, TX

TX034 - SOUTHERN METHODIST UNIV SCIENCE LIBRARY, TX

TX018 - TEXAS A\&M REMOTE SENSING CENTER, TX

TX025 - TEXAS A\&M UNIVERSITY, CHEMISTRY DEPT, TX

TX003 - TEXAS AIR CONTROL BOARD, TX

TX022 - TEXAS COASTAL AND MARINE COUNCIL, TX

TXO10 - TEXAS DEPARTMENT OF AGRICULTURE, TX

TX006 - TEXAS DEPARTMENT OF HEALTH, TX

TX001 - TEXAS DEPARTMENT OF WATER RESOURCES, TX

TXOII - TEXAS DEPT-HIGHWAYS \& PUBLIC TRANSPORTATION, TX 
TX004 - TEXAS FOOREST SERVICE, TX

TX002 - TEXAS GENERAL LAND OFEICE, TX

TX005 - TEXAS INDUSTRIAL COMMISSION, TX

TX015 - TEXAS NATURAL RESO INFORMATION SYSTEM, TX

TX012 - TEXAS PARKS \& WILDLIFE DEPARTMENT, TX

TX013 - TEXAS STATE SOIL \& WATER CONSERVATION BOARD, TX

TX040 - THOMAS G. GEBHARD, CONSULTING ENGINEER, TX

TX031 - TOBIN RESEARCH, TX

TX021 - UNITED AERIAL MAPPING, TX

TX027 - WILLIAMS-STACKHOUSE INC AERIAL MAP MOSAICS, TX

TX042 - WILSON AERIAL SURVEYS, TX

\section{UTAH}

UTO16 - CENTER FOR WATER RESO RESE, UTAH STATE UNIV, UT UTO07 - CLEAR LAKE WATERFOWL MANAGEMENT AREA, UT UT003 - DIV-FISH \& GAME, UTAH DEPT OF NAT RESOURCES, UT UT013 - DIV OF WATER RIGHTS, UTAH DEPT OF NAT RESO, UT UTO02 - METROPOLITAN WATER DIST OF SALT LAKE CITY, UT UTOI4 - MOUNTAINLAND ASSOCIATION OF GOVERNMENTS, UT UTO06 - OGDEN BAY WATERFOWL MANAGEMENT AREA, UT UTOO9 - OGDEN RIVER WATER USERS, UT UTO05 - SALT LAKE CITY WATER SUPPLY \& WATERWORKS, UT UTO12 - SALT LAKE COUNTY RECREATION DEPARTMENT, UT 
ORGANI -

ZATION

CODE

ORGANI ZATION NAME

UTO04 - SALT LAKE COUNTY WATER CONSERVANCY DISTRICT, UT UTOI5 - TEMPLETON, LINK \& ALSUP ASSOCIATION, UT UTOI7 - UINTEX CORPORATION, UT UTO18 - UTAH DEPARTMENT OF TRANSPORTATION, UT UTO08 - UTAH GEOLOGICAL \& MINERALOGICAL SURVEY, UT UT001 - UTAH STATE HEALTH DEPARTMENT, UT UTOII - UTAH WATER RESEARCH LAB, UTAH STATE UNIV, UT UTOIO - WEBER DISTRIBUTION SYSTEM, UT

\title{
VERMONT
}

VTO01 - STATE OF VERMONT, AGCY OF ENVIR CONSERVATION, VT VTO07 - VERMONT AGENCY OF TRANSPORTATION, VT VTO05 - VERMONT DEPARTMENT OF FISH AND GAME, VT VTO02 - VERMONT DEPARTMENT OF HEALTH, VT VTO04 - VERMONT DEPARTMENT OF WATER RESOURCES, VT VTO03 - VERMONT WATER RESO RESE CTR, UNIV OF VERMONT, VT VT006 - UNIVERSITY OF VERMONT, VT

\author{
VIRGIN I SLANDS (US)
}

VQ00I - VIRGIN ISLANDS DEPARTMENT OF PUBLIC WORKS, VI

\section{VIRGINIA}

VA009 - AIR-PHOTOGRAPHICS, INC, VA 
VA011 - AIR SURVEY \& DESIGN, INC, VA

VA005 - FAIRFAX CNTY (LOWER POTOMAC) TREATMENT PLANT, VA VA010 - JRB ASSOCIATES, INC, VA

VA004 - OCCOQUAN WATERSHED MONITORING LAB, VA

VA012 - SCS ENGINEERS, VA

VA013 - VA DEPT OF AGR \& CONSUMER SERVICES, VA

VA007 - VA DEPT OF HIGHWAYS AND TRANSPORTATION, VA

VA008 - VERSAR (SPRINGFIELD), VA

VA003 - VIRGINIA MILITARY INST RESEARCH LABORATORIES, VA VA002 - VIRGINIA STATE BUR OF GAME \& INLAND FISHERIES,VA VA001 - VIRGINIA STATE WATER CONTROL BOARD, VA VA006 - VIRGINIA POLYTECHNIC INST \& STATE UNIV, VA

\section{WASHI NGTON}

WA021 - ALBROOK LABORATORY, WASHINGTON STATE UNIV, WA WA032 - BATTELLE PACIFIC NORTHWEST LAB, WA WA027 - BURLINGTON NORTHERN, WA WA003 - CHELAN COUNTY PUBLIC UTILITY DISTRICT NO 1, WA WA030 - CITY OF BELLEVUE, SURVEY DEPT, WA WA007 - CITY OE BREMERTON WATER DEPARTMENT, WA WAO08 - CITY OF EVERETT DEPARTMENT OF WATER, WA WA028 - CLARK COUNTY MAP SERVICE, WA WAOI7 - DEPARTMENT OF LIGHTING, CITY OF SEATTLE, WA 


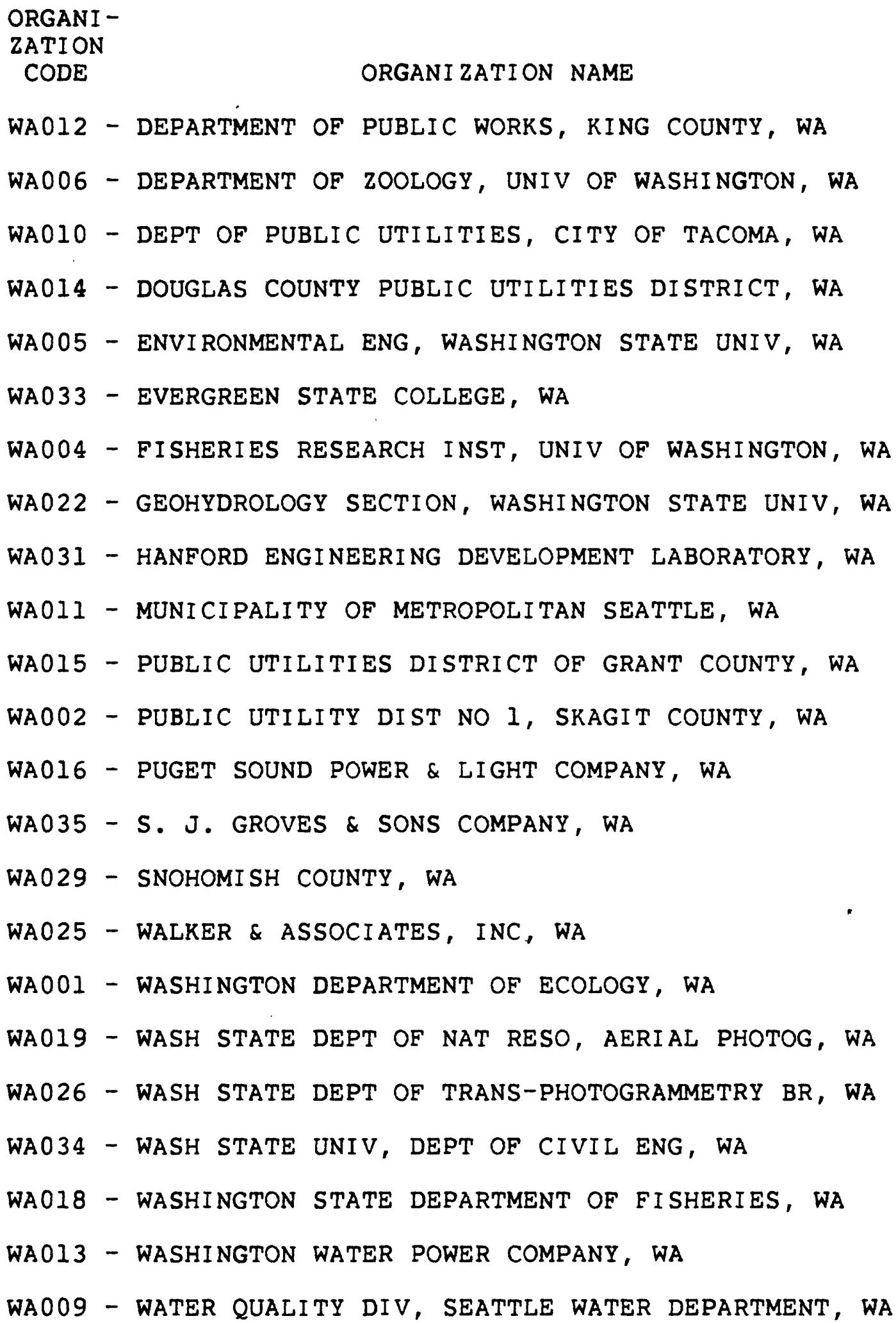


ORGANI -

ZATION

CODE

ORGANI ZATION NAME

WA020 - WATER RESEARCH CENTER, WASH STATE UNIV, WA

WA024 - WEYERHAEUSER COMPANY, WA

WEST VIRGINIA

WV001 - DIV OF WATER RESO, W VA DEPT OF NAT RESO, WV

WVO02 - SANITARY ENG DIV, W VA DEPARTMENT OF HEALTH, WV

WV003 - W VA GEOLOGICAL \& ECONOMIC SURVEY, WV

WV004 - WEST VIRGINIA DEPARTMENT OF HIGHWAYS, WV

WV005 - WEST VIRGINIA ENVIRONMENTAL HEALTH SERVICE, WV

WI SCONSIN

WIOII - BAY-LAKE REGIONAL PLANNING COMMISSION, WI

WI003 - DAIRYLAND POWER COOPERATIVE, WI

WIOIO - DANE COUNTY REG PLANNING COMMISSION, WI

WI002 - EAST CENTRAL WISCONSIN REGIONAL PLANNING COM, WI

WIOI7 - ENVIRO ENERGY TECHNOLOGY CENTER (REXNORD), WI

WIO04 - NORTHERN STATES POWER COMPANY, WI

WI0I6 - REMOTE SENSING DATA CENTER, UNIV OF WIS, WI

WI008 - SOUTHEASTERN WISCONSIN REG PLANNING COMM, WI

WI0I5 - STATE CARTOGRAPHER'S OFFICE, UNIV OF WISC, WI

WI007 - UNIV OF WIS, COLLEGE OF ENG, K F WENDT LAB, WI

WI0I2 - UNIVERSITY OF WISCONSIN SEA GRANT PROGRAM, WI 
ORGANI -

ZATION

CODE

ORGANI ZATION NAME

WIOOI - WISCONSIN DEPARTMENT OF NATURAL RESOURCES, WI

WI0I4 - WISCONSIN DEPARTMENT OF TRANSPORTATION, WI

WI009 - WISCONSIN GEOLOGICAL SURVEY, WI

WI006 - WISCONSIN PUBLIC SERVICE COMMISSION, WI

WI005 - WISCONSIN STATE LABORATORY OF HYGIENE, WI

WYOMING

WY00I - CITY OF CASPER BOARD OF PUBLIC UTILITIES, WY

WY002 - SHERIDAN WATER DEPARTMENT, WY

WY004 - WATER RESOURCES RESE INST, UNIV OF WYOMING, WY WY006 - WYOMING DEPARTMENT OF ENVIRONMENTAL QUALITY, WY WY005 - WYOMING HIGHWAY DEPARTMENT, WY WY003 - WYOMING STATE ENGINEER, WY 